\title{
Overview of the Space Launch System Transonic Buffet Environment Test Program
}

\author{
David J. Piatak ${ }^{1}$ Martin K. Sekula ${ }^{2}$ Russ D. Rausch ${ }^{3}$ James R. Florance $^{4}$ Thomas G. Ivanco ${ }^{5}$ \\ Aeroelasticity Branch / NASA Langley Research Center \\ Hampton, VA 23681
}

\begin{abstract}
Fluctuating aerodynamic loads are a significant concern for the structural design of a launch vehicle, particularly while traversing the transonic flight environment. At these trajectory conditions, unsteady aerodynamic pressures can excite the vehicle dynamic modes of vibration and result in high structural bending moments and vibratory environments. To ensure that vehicle structural components and subsystems possess adequate strength, stress, and fatigue margins in the presence of buffet and other environments, buffet forcing functions are required to conduct the coupled loads analysis of the launch vehicle. The accepted method to obtain these buffet forcing functions is to perform wind-tunnel testing of a rigid model that is heavily instrumented with unsteady pressure transducers designed to measure the buffet environment within the desired frequency range. Two wind-tunnel tests of a 3-percent scale rigid buffet model have been conducted at the Langley Research Center Transonic Dynamics Tunnel (TDT) as part of the Space Launch System (SLS) buffet test program. The SLS buffet models were instrumented with as many as 472 unsteady pressure transducers to resolve the buffet forcing functions of this multi-body configuration through integration of the individual pressure time histories. This paper will discuss the test program development, instrumentation, data acquisition, test implementation, data analysis techniques, and several methods explored to mitigate high buffet environment encountered during the test program. Preliminary buffet environments will be presented and compared using normalized sectional buffet forcing function root-mean-squared levels along the vehicle centerline.
\end{abstract}

\section{Nomenclature}

$\begin{array}{ll}A & =\text { Area } \\ \text { AB-DAS } & =\text { Aeroelasticity Branch Data Acquisition System } \\ \text { BFF } & =\text { buffet forcing function } \\ \text { BMO } & =\text { buffet mitigation option } \\ \text { CFD } & =\text { computational fluid dynamics } \\ C_{p} & =\text { pressure coefficient } \\ D & =\text { diameter } \\ \text { DAS } & =\text { data acquisition system } \\ \text { ESP } & =\text { electronically scanned pressure } \\ f & =\text { frequency, Hz } \\ F & =\text { force } \\ \text { FRF } & =\text { frequency response function } \\ f S & =\text { full-scale quantity } \\ \text { ICPS } & =\text { Interim Cryogenic Propulsion Stage } \\ L & =\text { length } \\ \text { LAS } & =\text { Launch Abort System } \\ \text { LSRB } & =\text { left solid rocket booster }\end{array}$

\footnotetext{
${ }^{1}$ Research Aerospace Engineer, Aeroelasticity Branch, david.j.piatak@nasa.gov.

${ }^{2}$ Research Aerospace Engineer, Aeroelasticity Branch, martin.k.sekula@nasa.gov.

${ }^{3}$ Assistant Head, Aeroelasticity Branch, russ.d.rausch@nasa.gov, Associate Fellow AIAA

${ }^{4}$ Research Aerospace Engineer, Aeroelasticity Branch, james.r.florance@nasa.gov, Senior Member.

${ }^{5}$ Research Aerospace Engineer, Aeroelasticity Branch, thomas.g.ivanco@nasa.gov, Senior Member.
} 


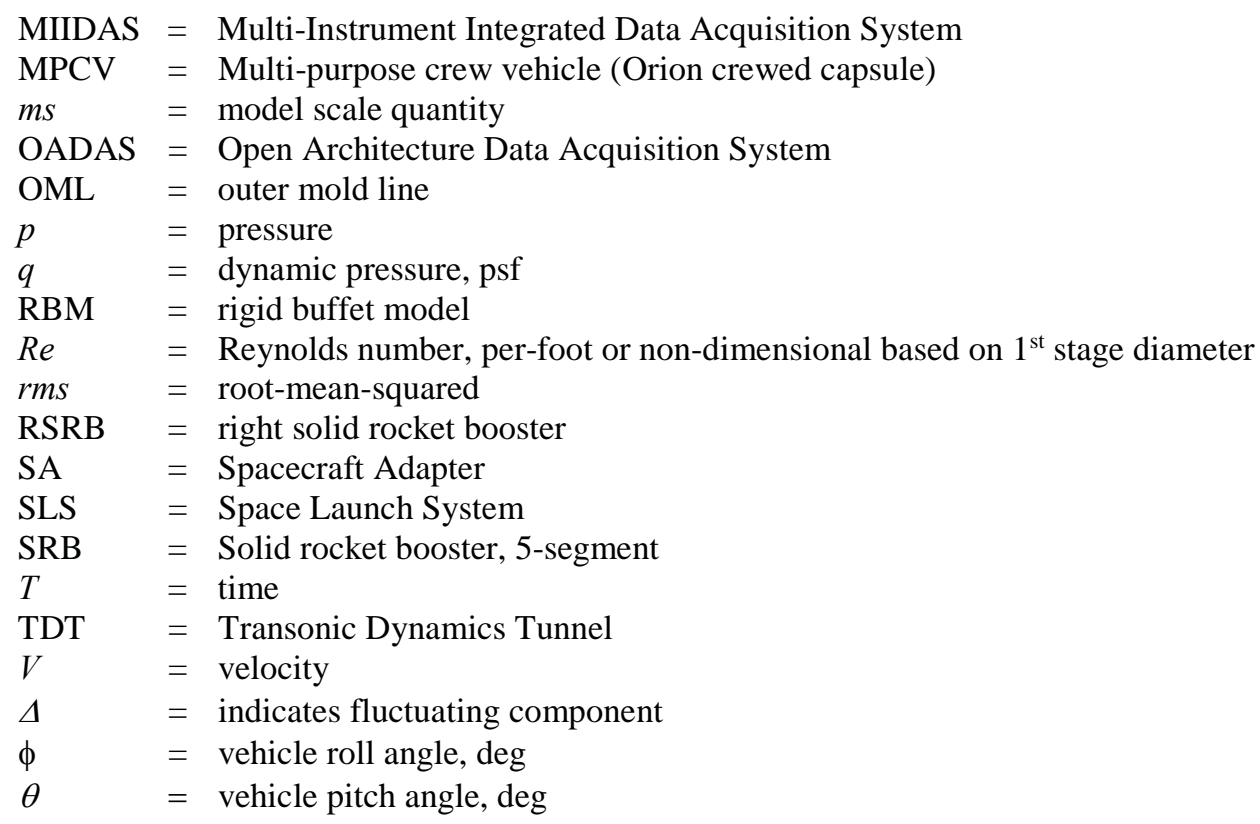

\section{Notice to Readers}

The predicted performance and certain other features and characteristics of the Space Launch System vehicle are defined by the U.S. Government to be Sensitive But Unclassified (SBU). Therefore, values in plots and figures have been either removed or normalized to arbitrary values.

\section{Introduction}

T aunch vehicle buffet environments consist of unsteady aerodynamic phenomenon dominated by fluctuating

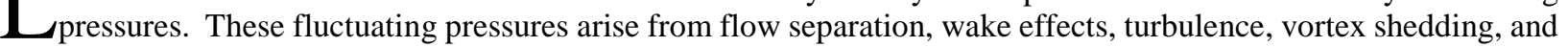
transonic shock oscillations. This aerodynamic environment is further complicated by rapidly changing trajectory conditions and vehicle configurations which include large payload shrouds, multi-body boosters, and spacecraft/payload interface adapters. Vehicle structural modes will be excited by the fluctuating pressures associated with the buffet environment and the resulting buffet loads can be a significant fraction of overall vehicle structural loads, particularly in the transonic regime. Figure 2 illustrates the complex unsteady aerodynamic environment and notional structural deflections associated with the Space Launch System (SLS) configuration. Launch vehicle buffet loads analyses are typically concerned with pressure fluctuations and vehicle structural responses below $60 \mathrm{~Hz}$. Higher frequency vibratory responses are considered part of the vibro-acoustic loads discipline. ${ }^{1-3}$

Great progress in time-accurate computational fluid dynamics (CFD) codes have been accomplished in recent years and such tools have been used to understand key aspects of unsteady flow phenomena. Excellent comparisons to experimental unsteady pressure time histories have been shown and have been used to guide the direction of the SLS buffet test program. However, time-accurate CFD solutions remain too costly and time-intensive to be used to adequately define the buffet environment for a complex launch vehicle given the multitude of configurations and flight conditions typically required. Today, the accepted method to determine launch vehicle buffet environments is to perform wind-tunnel testing on a rigid buffet model with an adequate spatial distribution of unsteady pressure sensors to resolve the fluctuating pressure environments at discrete locations on the vehicle surface. ${ }^{4}$ These discrete fluctuating pressures, typically 100s of them, are then utilized to determine buffet forcing functions (BFFs) at numerous longitudinal stations at the centerline of the vehicle. ${ }^{5-7}$ The BFFs consist of orthogonal force time histories at each station and are used as inputs to a finite-element based structural analyses to determine the vehicle response and resulting buffet loads and accelerations. ${ }^{8}$ These buffet loads and responses are then summed together with the results from other launch vehicle environments as part of a coupled loads analysis approach to assure that the vehicle can withstand the applied combined loads and subsequent vehicle responses during its flight trajectory. Previous work using the aforementioned buffet test and analysis techniques have proven accurate when compared to measured flight data on the Ares I-X flight test vehicle. ${ }^{9}$

Two wind-tunnel tests were conducted at the Langley Transonic Dynamics Tunnel (TDT) on a 3-percent scale rigid buffet model of numerous SLS vehicle configurations for the purpose of defining the buffet environments for 
input to the vehicle structural design. During testing in the fall of 2012, time-correlated unsteady pressures were obtained at 360 locations for the SLS-10003 Block I 70-metric-ton MPCV configuration, the SLS-11000 Block IB 93-metric-ton with 8.4 meter payload shroud configuration, and the SLS-13000 Block IB MPCV with secondary payload shroud configuration. These configurations are shown in Figure 1. Results from the 2012 test revealed higher-than-expected buffet environments in the region downstream of the solid rocket booster (SRB) forward attachment location, between the left and right SRBs and the core stage. These high buffet environments resulted in the formation of a cross-organizational team targeted at determining buffet mitigation options (BMOs) that might alleviate the buffet loads and improve vehicle structural margins. Several BMOs were put forward which proved promising in time-accurate CFD computations and were tested during the SLS ascent aeroacoustic test at the NASA Ames 11-ft Unitary Plan Wind Tunnel (UPWT). These BMOs included multiple SRB nose cone shapes and fences placed on the core stage and SRBs in the vicinity of the booster forward attachments. Results from the Ames windtunnel test were used to down-select BMOs for further buffet testing at the LaRC TDT.

The second SLS buffet program wind-tunnel test at the TDT was conducted in spring/summer of 2014. The primary goal was to obtain higher fidelity buffet environments and forcing functions for the updated outer mold line (OML) of the SLS-10005 Block I 70-metric-ton MPCV configuration. Emphasis was placed on increased sensor spatial resolution and understanding of sensor-to-sensor spatial correlation in the booster forward attachment regions. To achieve this, the 3-percent scale SLS buffet model was modified to include additional sensors to bring the total number of unsteady pressure measurement locations to 472, far beyond any previous launch vehicle buffet investigation. Additionally, all vehicle external protuberances were updated to reflect the current vehicle configuration including a detailed representation of the booster forward attachment hardware.

This paper will discuss the test development, test implementation, data analysis methodologies, and buffet environments associated with the SLS buffet test program. Time-correlated fluctuating pressures were obtained at up to 472 locations on the model with adequate spatial resolution to capture the challenging multi-body buffet environments of the SLS configurations. A subset of the pressure measurements was acquired at significantly higher bandwidths and were located at locations to support verification of the SLS aeroacoustic environments. Test conditions were focused on the transonic environments between Mach 0.8 and 1.2. The vehicle's flight orientation was simulated by pitching the model between $+/-8$ degrees and rolling between $+/-180$ degrees. Comparisons of $\Delta C_{p, r m s}$ will be presented to illustrate trends in buffet environments across the two TDT tests and across the various configurations tested.

\section{Test Objectives}

The primary test objective is the acquisition of time-correlated unsteady pressure data with sufficient spatial resolution to define the transonic buffet environment on the multi-body SLS vehicle for multiple configurations. Pressure time histories acquired during testing must be at scan rates adequate to satisfy a full-scale bandwidth of at least $60 \mathrm{~Hz}$. The secondary test objective of the buffet test program was to obtain aeroacoustic pressure time histories at a scan rate equivalent to $2,000 \mathrm{~Hz}$ full-scale for a limited subset of the sensor locations. These buffet pressure time histories are to be spatially integrated to yield centerline buffet forcing functions in three orthogonal directions, thus the importance that all measurements be time-correlated and at sufficient scan-rates.

\section{Test Facility}

The NASA Langley Transonic Dynamics Tunnel is a closed-circuit, continuous flow wind tunnel conceived in the late 1940's and early 50's for the unique purpose of conducting aeroelastic testing of a wide variety of flight vehicles. Past launch vehicle buffet test programs conducted at the TDT have included Apollo-Saturn, Atlas-Centaur, Ares IX, Ares I, and Dream Chaser-Atlas V. The TDT has a 16-foot-by-16-foot slotted transonic test section capable of reaching Mach 1.2 at a range of stagnation pressures from near vacuum to atmospheric pressure in both an air and R134a heavy gas test medium. Dynamic pressures up to 300 psf and 550 psf can be attained in air and R134a, respectively. ${ }^{10-12}$ Table 1 lists the TDT test conditions for several Mach numbers for the SLS buffet model testing.

Testing in a heavy gas is advantageous to test programs where time and frequency scaling are important, such as for launch vehicle buffet and aeroacoustic testing. Since the speed of sound in R134a is approximately half that in air, model-scale frequencies in R134a are also half those in air, resulting in more favorable bandwidth requirements for instrumentation and data systems. Model-scale and aeroacoustic bandwidths provided in Table 1 are trajectory dependent. Table 2 presents buffet model scaling relationships used to determine model-scale frequency bandwidths. 


\section{Model Design}

The choice of model geometric scale factor is one of the first parameters to be considered during model design. Rigid buffet models are typically much larger than a simpler force and moment wind tunnel models due to the large amount of instrumentation required to fit into a buffet model. For the SLS buffet test program, the model possessed 360 and 472 unsteady pressure transducers, respectively, for the tests conducted in 2012 and 2014. The associated cabling, power distribution, and pressure tubing for all of the transducers presents a packaging and model assembly challenge which drives aspects of the model design, requiring a larger scale model. The SLS rigid buffet model design benefited from the experience of the Ares I and Dream Chaser -Atlas V buffet test programs and employed a "clamshell" component design. The assembly components of the model are illustrated in Figure 3. Major components are shown such as the launch abort system (LAS), the multi-purpose crew vehicle (MPCV), the interim cryogenic propulsion system (ICPS), the right and left solid rocket boosters (LSRB/RSRB), core stage, core base, and the sting support. Each major component was comprised of two halves which allowed for installation of instrumentation. Besides providing ample interior space for instrumentation, a larger model is also advantageous from the standpoint of time and frequency scaling since the model-scale frequency bandwidth is inversely proportional to the model geometric scale factor. This relationship yields a lower model-scale bandwidth requirement for unsteady pressure transducers and data acquisition systems. Another consideration in choosing the geometric scale factor for a launch vehicle wind-tunnel model is the facility test section length. The overall length of the constant Mach number region in the TDT test section ranges from approximately 30 feet at and below Mach 1 and reduces to approximately 15 feet at Mach 1.2, which dictated a model scale factor of three percent.

\section{A. 2012 SLS Buffet Test Configurations}

Three primary configurations were tested during the 2012 test entry. These included the SLS-10003 Block I 70metric-ton MPCV configuration, the SLS-11000 Block IB 93-metric-ton with 8.4 meter payload shroud configuration, and the SLS-13000 Block IB MPCV with secondary payload shroud configuration. (Note: the configuration nomenclature listed above represents the vehicle as defined in early 2012; vehicle design has evolved since then, thus differences in vehicle naming conventions may be found in present NASA literature.) For each configuration, 296 buffet bandwidth unsteady pressure orifices, 64 aeroacoustic pressure orifices, and 64 static pressure orifices were included in the design. The majority of the 64 aeroacoustic sensors were dual-use buffet/aeroacoustic measurements. Figure 1 illustrates the three configurations tested during the 2012 test entry. The shape of the forward booster/core attachment protuberance was simplified for the 2012 test due to fact that it was not well defined at that point in the SLS program design. Figure 4 illustrates the differences between the simplified booster forward attachment tested in 2012 and the detailed OML shape testing in 2014. Figures 5 through 9 present photos of the 2012 SLS rigid buffet model in the TDT test section.

\section{B. 2014 SLS Buffet Test Configurations}

Prior to the 2014 SLS buffet test, the model was extensively modified to include an additional 112 transducer orifice locations for increased azimuthal and longitudinal spatial resolution in the vicinity downstream of the booster forward attachment protuberance. Figure 10 illustrates the additional longitudinal measurement stations and which stations had additional azimuthal sensor locations. The fidelity of the booster forward attachment protuberance was increased and all other protuberances updated to represent the most up-to-date design OML. During post-test analysis of the 2012 test data, the buffet and acoustic environments downstream of the booster forward attachment protuberance yielded very high $\Delta C_{p, r m s}$ levels on the core and booster regions. This led to high induced buffet loads on the vehicle that warranted closer study due to the reduced structural margins observed as part of vehicle coupled loads analysis.

The high buffet environments triggered a significant study of the flow phenomenon using CFD to guide buffet mitigation options (BMOs). Two categories of BMOs were developed which centered on a fence or strake on the booster or core. Another option involved modifying the booster nose cones in attempts to eliminate or reduce the high fluctuating pressure magnitudes between booster and core stage. Both categories of BMOs were tested at the NASA Ames 11-foot UPWT wind tunnel as part of an SLS ascent aeroacoustic test in 2013. The BMOs which yielded the most significant reductions in buffet environments were then included in the re-design of the SLS rigid buffet model. It was also felt that the increased spatial resolution of transducers would improve the understanding of azimuthal and longitudinal coherence which is used to generate "coherence factors" which reduce the integration regions and inherent conservatism of discrete sensor orifices when generating buffet forcing functions.

There were six fundamental configurations tested during the 2014 SLS rigid buffet test. These included the baseline SLS-10005 configuration with updated protuberances and increased sensors, booster fences, core fence, and three nose cones referred to as canted ogive, bent bi-conic, and canted straight noses. Figure 11 shows the SLS-10005 
rigid buffet model installed in TDT test section and Figure 12 shows the detailed booster forward attachment protuberance. Updated liquid oxygen (LOX) feedline details from the top view (zero degree azimuth) perspective are shown in Figure 13. Figures 14 through 16 show the booster and core fence configurations tested. Baseline, canted ogive, bent bi-conic, and canted straight nose cone configurations are shown in Figures 17 through 20. Updated gaseous oxygen (GO2) and gaseous hydrogen (GH2) pressurization lines and cable trays are also shown in Figure 18 from the bottom perspective view of model (180 degree azimuth).

\section{Model Instrumentation}

The measurement of buffet and acoustic environments on a wind-tunnel model requires careful attention to instrumentation design and data acquisition systems to assure that the data is acquired at the proper model-scale scan rates to meet full-scale bandwidth requirements. Equivalent model-scale bandwidth requirements were determined using the relations shown in Table 2. The resulting scale relationships are dependent upon the flight vehicle trajectory, tunnel conditions, and model geometric scale. Using the relations in Table 2, the desired bandwidths for buffet and aeroacoustic data are approximately $1.1 \mathrm{KHz}$ and $35 \mathrm{KHz}$ respectively. Specific required bandwidths for different flight/tunnel conditions are shown in Table 1. The choice of pressure sensor, amplification, and installation design can have a large impact on the ability to satisfy program requirements. The instrumentation design must keep the ultimate deliverable of the test program in mind, that is, the centerline buffet forcing functions. These forcing function time histories are generated by careful integration of individual sensor pressures and analysis of sensor-to-sensor coherence, which is a quantification of how alike two pressure time histories are in frequency content. Buffet test requirements typically dictate a large number of unsteady pressure transducers to provide reasonable spatial resolution for pressure integration and coherence analysis. In additional to the unsteady pressure measurements, model vibration was measured using piezoresistive accelerometers and model orientation (pitch/roll) was measured using highly sensitive Q-flex accelerometers. During the 2012 SLS buffet test, 64 static pressures were measured using a Pressure Systems Inc. series 8400 electronically scanned pressure (ESP) system.

Three custom-designed unsteady pressure transducers were supplied by Kulite Semiconductor Products, Inc. for use in the SLS test program. Table 3 lists the Kulite model pressure transducers installed in the SLS rigid buffet model. Each transducer includes an in-line amplifier with voltage regulated power circuitry to ensure minimal attenuation of the signal over the long cable run to the data acquisition system and to ensure proper voltage supply to the transducer. XCL-100 type transducers were used for buffet measurements at most orifice locations on the model. Where interior space was limited such as within the LAS and tips of SRB nose cones, smaller type XCL-072 transducers were used. The XCL-100 and -072 buffet transducer types had a 3KHz usable bandwidth to satisfy SLS test requirements of a minimum of $1 \mathrm{KHz}$ model-scale bandwidth. All acoustic measurement orifices were populated with XCL-072 with an amplifier and transducer design that yields a $57 \mathrm{KHz}$ bandwidth, well outside of the $35 \mathrm{KHz}$ model-scale bandwidth requirement.

The installation design for the transducers should take into account the need to have minimal effect on the flow at surface of model OML. Attempting to flush-mount a transducer sensor face to the model OML where curvature is present will result in a partially recessed transducer or one that partially protrudes beyond the defined OML. An orifice at the model surface is the best choice that minimizes transducer installation effects on the measured pressure. Past experience with unsteady pressure measurements dictates a 0.040 -inch diameter orifice, beneath which a transducer is mounted. However, the length of this orifice and any cavity between the transducer face and model OML will result in a cavity resonance sometimes referred to as Helmholtz resonance. This cavity resonance must be taken into account in the design of the transducer installation and can be calculated using formulas found in reference 13. It is typically accepted that the cavity resonance frequency should be at least five times the maximum frequency of interest of the unsteady pressure to be measured. Otherwise, the cavity resonance-induced rise in frequency response between the pressure time history observed at the model surface and that observed by the transducer below the orifice/cavity will lead to an erroneously high pressure time history amplitude. Figures 21 and 22 illustrate the design geometry of the typical buffet and acoustic transducer installation and dimensional information regarding this geometry is listed in Table 4. Each transducer is sealed into the barrel using an RTV sealant, which allows for relatively easy removal while still assuring an air-tight seal between the transducer face and the orifice. Using this geometry, the cavity resonance was computed for each transducer installation and is listed in

Table 5. The computed cavity resonance is sufficiently high to meet the factor of five guideline for the buffet bandwidth. However, meeting the factor of five criteria for acoustic bandwidth proved to be a challenge, but best efforts were made in the design for the acoustic inserts shown in Figure 22.

Each Kulite transducer was a differential measurement referenced to the TDT static pressure. Thus, each Kulite transducer had a back-side reference tube that was connected to manifolds which provided the common back-side 
pressure to all Kulites. This back-side reference pressure tubing was also used to apply calibration pressures to all transducers to allow for in-situ calibrations and pre- and post-run health checks of all unsteady pressure transducers in the model.

\section{Data Acquisition Systems}

Because of the channel count and acquisition rate requirements of the SLS buffet test program and the limitations of the facility data acquisition system (DAS), SLS buffet data was acquired using independent data acquisition systems at unique scan rates. For both the 2012 and 2014 tests, primary tunnel parameters, and model pitch/roll orientation was acquired by the facility DAS known as the Open Architecture Data Acquisition System (OADAS) that utilizes NEFF 620 hardware. In the 2012 test, the OADAS also acquired the steady ESP pressure data. OADAS acquired all data quantities with 10 -second data records at a scan rate of $500 \mathrm{~Hz}$ with anti-alias filters at $200 \mathrm{~Hz}$. The OADAS anti-alias filter was an analog 6-pole Bessel filter. Between the 2012 and 2014 tests, a new DAS development program was initiated to replace the OADAS at TDT with an entirely new system capable of acquiring large-channel-count and high-scan-rate synchronous data typical of buffet tests. Thus, the DAS differed between the 2012 and 2014 SLS buffet tests and the systems are discussed in detail below. Table 6 contains a compilation of model instrumentation categorized by type, and it summarizes the scan rates and DAS utilized to acquire data.

\section{A. 2012 Data Systems}

In addition to the facility OADAS, buffet bandwidth pressure data and model response accelerometer data were acquired with the Multi-Instrument Integrated Data Acquisition System (MIIDAS) that utilizes NEFF 730 hardware. The MIIDAS system acquired 10-second data records at a scan rate of $12 \mathrm{KHz}$ with anti-alias filters at $4.5 \mathrm{KHz}$. The MIIDAS anti-alias filter configuration used an analog 8-pole Bessel filter coupled with a post-scan digital filter. All 360 unsteady pressure transducers were acquired by MIIDAS in the buffet bandwidth, out of which 64 were capable of aeroacoustic bandwidth. The aeroacoustic transducer data were also output by the MIIDAS signal conditioner as wideband (unfiltered) analog signals prior to digitization. The aeroacoustic wideband data were then acquired in parallel by the Piranha III Digital Dynamic Data Monitoring and Analysis System developed by DSPCon. The DSPCon system acquired the 64 aeroacoustic bandwidth pressure transducers for 10 -second data records at a scan rate of $100 \mathrm{KHz}$ with anti-alias filters at $45 \mathrm{KHz}$. The DSPCon anti-alias filter configuration used a 2-pole analog Butterworth filter at a fixed $320 \mathrm{KHz}$ cutoff frequency coupled with a Sigma-Delta digital filter with a cutoff frequency of 0.4535 times the scan rate $(45.35 \mathrm{KHz})$. Both the NEFF 730 and DSPCon systems acquired synchronous timecorrelated data; a necessity for buffet load analysis. Validation testing was done of both the MIIDAS and DSPCon data systems to validate the DC measurement accuracy, dynamic measurement accuracy with regard to magnitude and phase shift, and performance of the anti-alias filters.

\section{B. 2014 Data Systems}

In addition to the facility OADAS, all buffet and aeroacoustic pressure data, accelerometer data, Q-flex model orientation data, and digital tunnel parameter data acquired for the 2014 SLS buffet test entry were recorded using the Aeroelasticity Branch Data Acquisition System (AB-DAS). AB-DAS is intended to become the primary facility DAS at TDT, and is based on National Instruments (NI) PXIe A-to-D data acquisition chassis and boards and Precision Filter Incorporated (PFI) 28000 series signal conditioners. The AB-DAS buffet bandwidth system acquired 20-second data records at a scan rate of $16 \mathrm{KHz}$ with anti-alias filters at $6 \mathrm{KHz}$. All 472 unsteady pressure transducers were acquired by AB-DAS in the buffet bandwidth, out of which 32 were capable of aeroacoustic bandwidth. The aeroacoustic transducer data were also output by the PFI signal conditioners as wideband (unfiltered) analog signals. The aeroacoustic wideband data were then acquired in parallel by a second AB-DAS system for 10-second data records at a scan rate of $200 \mathrm{KHz}$ with anti-alias filters at $60 \mathrm{KHz}$. The AB-DAS anti-alias filter configuration was an analog 4-pole flat filter that approximates a 4-pole Butterworth filter with slightly better performance. Similar to the other dynamic data systems, AB-DAS acquires synchronous time-correlated data as required for buffet load analysis. Validation testing was done of the AB-DAS system to validate the DC measurement accuracy, dynamic measurement accuracy with regard to magnitude and phase shift, and performance of the anti-alias filters as described in the following sections.

\section{Test Implementation}

\section{A. Instrumentation Calibration and Data Quality Checks}


Facility data systems and model transducers were checked out following installation of the model and instrumentation to verify functionality, calibration, patching and channel labeling, and over-all performance. Instrumentation checkout testing included: front-side port leak checks, back-side reference tube and manifold leak checks, channel/transducer identification end-to-end checks, transducer calibration, and verification of calibration constants by applying a known front-side port pressure. While the procedures were similar between the 2012 and 2014 SLS Buffet Tests, the data systems used and their configurations were different as described in the previous section. For both tests, the pressure transducer calibrations were carried out using an ESP system Pressure Calibration Unit to apply ten or more steady pressures to the back-side reference tube of each pressure transducer. The subsequent response voltages were measured using the previously mentioned data acquisition system configurations employed for each test. A fifth-order polynomial curve fit was then applied to the calibration data to obtain engineering unit conversion constants.

Model position was measured by three orthogonally mounted Q-flex accelerometers which were calibrated using the NASA Langley Angle Measurement System with the model installed in the TDT. The model was pitched and rolled through the angles of interest, and the response voltages of the Q-flex accelerometers were recorded using the TDT OADAS for both the 2012 and 2014 SLS buffet tests and also by the AB-DAS for the 2014 test. The engineering unit conversion constants were computed using an appropriate curve fit applied to the calibration data to yield model pitch and roll (ref. 14) and sting angle of attack.

The frequency response of the Kulite pressure transducers were checked in-situ by applying white noise sound pressure to a number of model pressure ports using a speaker driver with a specialized test cap containing a 0.125inch reference microphone (Figures 23 and 24). The microphone and Kulite sensor signals were acquired simultaneously to allow for frequency response functions to be computed for a sampling of the model Kulites. Due to the large number of Kulite sensors, the inaccessibility of certain sensor locations, and experience from previous work with similar sensors, not all Kulite sensors were checked, but rather a representative sampling was deemed sufficient. Figure 25 presents a representative buffet and aeroacoustic bandwidth Kulite frequency response functions measured in-situ within the SLS RBM. Frequency response is shown to be within $\pm 1 \%$ of input signal at $1 \mathrm{KHz}$ model-scale and has a phase shift of less than five degrees, which is more than sufficient to derive the buffet pressure time histories.

In addition to the instrumentation quality checks, the supplemental data systems used in conjunction with OADAS were also evaluated to ensure that the signal conditioning, filters, and channel scanning was working to the high degree expected. Similar procedures were used in 2012 to evaluate the performance of the MIIDAS and DSPCon systems as those used in 2014 to evaluate the performance of the AB-DAS system prior to acquiring test data. The details described here pertain to those verifications performed for the AB-DAS system. Calibrated signal sources (precision constant voltage, single frequency sine wave, and white noise) were applied to the calibration bus on each PFI chassis, subjected to the amplifier settings, and recorded through the NI hardware and software. Example data is shown in Figure 26 for a white noise signal banded between 1 and $10 \mathrm{KHz}$ input into the calibration bus of each PFI chassis and recorded across both AB-DAS systems for a total of 512 channels. From this data, frequency response between the first channel and each subsequent channel was determined. Since the identical signal is recorded on all channels via the calibration bus, the frequency response functions (FRFs) should show a constant magnitude of one and a phase shift of zero. However, minute differences in amplifiers, filters, and analog-to-digital conversion circuitry and settings can be observed. Gross differences in FRFs would indicate DAS failures or improper settings and could then be easily corrected. The FRF mean magnitudes between the first channel and all others are shown to be near 1.001 which is an excellent result and shows that signal conditioning and data acquisition components are operating nominally and exceed the performance of legacy TDT dynamic data systems. The mean values of FRF phase shown in Figure 26 are all less than one degree of phase shift. However, the larger increase in FRF phase at channel 244 corresponds to the split between NI data acquisition boards on the master PXIe chassis and slave chassis. This was to be expected and is well within limits of the requirements for SLS buffet testing and TDT dynamic data systems.

\section{B. Test Conditions and Procedures}

Buffet testing of the SLS RBMs was focused on the transonic regime and was conducted at Mach numbers of 0.70, $0.80,0.82,0.84,0.85,0.86,0.88,0.90,0.92,0.94,0.95,0.96,0.98,1.00,1.05,1.10,1.15$, and 1.20 . Dynamic pressure was typically the highest attainable at the test facility, with limited testing conducted at lower dynamic pressures to determine Reynolds number effects on buffet pressures. Table 1 lists TDT test section flow conditions (Reynolds number based on model first stage diameter) for typical RBM test matrix conditions and target model-scale bandwidth requirements. For the SLS-10000 configuration, data were acquired with air as the test medium for Mach numbers of $0.8,0.85,0.9,0.95$, and 1.2 . All other configurations were only tested with heavy gas as the test medium. The SLS10005 configurations with the three nose cone buffet mitigation options were tested at a reduced set of Mach numbers 
$\left(0.7,0.8,0.85,0.9,0.92,0.94,1.05,1.1,1.15\right.$, and 1.2). Model pitch orientation was set from $-6^{\circ}$ to $+6^{\circ}$ in $2^{\circ}$ increments, as well as at $+1^{\circ}$ and $-1^{\circ}$ for the 2012 test. For the 2014 test, model pitch angles were expanded to also include $-8^{\circ}$ and $+8^{\circ}$ to obtain data at abort conditions. Model roll orientation was set from -180 to $+180^{\circ}$ in $15^{\circ}$ increments during both tests. During the 2012 Test, the sensitivity of buffet loads to vehicle protuberances was determined via testing of both the clean (no protuberances) and the protuberance-on configurations discussed previously in the Model Design section.

For both the 2012 and 2014 SLS buffet tests, a pitch-pause technique was used where the model orientation was set, the tunnel flow conditions were stabilized, and then data were acquired. Multiple data systems were used as previously described to acquire the various bandwidths of data. Additionally, shadowgraphs of the transonic/supersonic flow-field were acquired using a high-intensity light source and video cameras to aid in the identification of flow phenomena during both tests.

\section{Buffet Environments and Comparisons}

Launch vehicle buffet environments will be presented as the root-mean-squared (rms) value of the fluctuating component of pressure coefficient, $\Delta C_{p, r m s}$. That is, the mean of the pressure signal is removed prior to computing the root-mean-squared value. Thus, $\Delta C_{p, r m s}$ indicates the degree of fluctuating buffet pressures due to shock oscillations, Strouhal shedding off protruding vehicle components, separated flow, unsteadiness due to flow reattachment, and other turbulent phenomena. The locations where $\Delta C_{p, r m s}$ is largest would indicate regions of the vehicle which are prone to high integrated buffet forcing functions at the vehicle centerline. These regions of high $\Delta C_{p, r m s}$ would likely excite the vehicle vibration modes and contribute to the overall vehicle buffet loads in the form of vibratory bending moments and accelerations.

Other metrics for defining and contrasting buffet environments include the frequency content of specific buffet pressure time history data and the sensor-to-sensor coherence magnitudes across frequency band of interest. These results for the SLS buffet test program are discussed in reference 15.

Multi-body configurations like the SLS pose a challenging buffet environment due to the interaction of flow structures in the vicinity of the SRB and core stage interface regions. Flow around the SRB nose cones can be quite asymmetric due to the presence of the core stage and the flow past the SRB forward attachment protuberance can exhibit Strouhal shedding, vortical flow, and "channeling" of the flow in this region between the core and booster. During initial buffet testing in 2012, high fluctuating pressure environments were observed just downstream of the SRB forward attachment protuberance. This triggered an effort to explore mitigation options to reduce these environments associated with the SRB forward attachment and these buffet mitigation options were employed on the SLS ascent aeroacoustic test at the NASA Ames 11-foot UPWT in the fall of 2013. These efforts culminated in the down-select of several promising BMOs for inclusion as part of the second test entry of the SLS buffet test program.

All results in this section are presented for a vehicle pitch and roll $(\theta, \phi)$ of zero degrees using an R134a heavy gas test medium to achieve maximum dynamic pressure and Reynolds number in TDT. All data are presented without defined numerical scales.

\section{A. 2012 Test Results}

Comparisons of $\Delta C_{p, r m s}$ for the SLS-10003, 11000, and 13000 configurations are presented in this section in Figures 27-42. Results for Mach 0.9 are shown in Figures 27 through 30 and show peak $\Delta C_{p, r m s}$ levels behind the simplified forward attachment protuberances on the core at azimuths $90^{\circ}$ and $270^{\circ}$ which face the boosters. The peak associated with the simplified forward attachment is also seen in Figure 29 on the LSRB at azimuths $225^{\circ}$ and in $315^{\circ}$ and in Figure 30 on the RSRB at azimuths $45^{\circ}$ and $135^{\circ}$ which face the core stage booster. Subtle differences in $\Delta C_{p, r m s}$ levels associated with the simplified forward attachment are seen between the three SLS configurations, with the SLS-11000 cargo configuration resulting in the highest peaks. Focusing on the forward region of vehicle configurations, Figure 27 shows differences in station location and magnitude of peak $\Delta C_{p, r m s}$ levels. This sensitivity in $\Delta C_{p, r m s}$ levels is due to the OML differences in the SLS-10003, 11000, and 13000 configurations which result in differences in terminal shock location and magnitude. The terminal shock peak is not observed on the core for the SLS-10003 and 13000 configurations in Figure 28 at azimuths 45/135/225/315 ${ }^{\circ}$ and is due to the fact that the sensor station locations are not as dense in this region at the $45 / 135 / 225 / 315^{\circ}$ azimuths for these configurations.

Results for Mach 0.95 are shown in Figures 31 through 34 and again show peak $\Delta C_{p, r m s}$ levels behind the simplified forward attachment protuberances on the core at azimuths $90^{\circ}$ and $270^{\circ}$ which face the boosters. The peak associated with the simplified forward attachment is also seen in Figures 33 and 34 on the LSRB and RSRB at azimuths which face the core stage booster. The peak $\Delta C_{p, r m s}$ level due to terminal shock location is not captured in Figure 31 or Figure 32, possibly due to fact that it falls between sensor locations. 
Supersonic results at Mach 1.10 and 1.19 on the three SLS configurations are presented in Figures 35 through 42 and show lower unsteady pressure fluctuations than the transonic conditions at Mach 0.90 and 0.95 . However, peak $\Delta C_{p, r m s}$ levels are still observed downstream of the simplified forward attachment at azimuths $90^{\circ}$ and $270^{\circ}$ which face the boosters. These peak levels are significantly lower in magnitude than at Mach 0.90 and 0.95, but still stand out within the overall buffet environment for these configurations.

The time-dependent buffet forcing functions integrated from the buffet environments summarized in this section resulted in high buffet loads and reduced vehicle structural margins, when applied to the vehicle structural finite element model. The outcome of the 2012 SLS buffet test program was to develop additional methodologies for generating the buffet forcing functions from raw pressure time histories and to pursue buffet mitigation options (BMOs) which may be used to reduce buffet environments.

\section{B. 2014 Test Results}

This section presents comparisons of $\Delta C_{p, r m s}$ values for the SLS-10005 vehicle with the detailed forward attachment (considered the baseline configuration for 2014 test), the simplified forward attachment (for comparison to 2012 baseline results), the sharp SRB fence, blunt SRB fence, core stage fence, canted ogive SRB nose cones, bent bi-conic SRB nose cones, and canted straight SRB nose cones.

\section{Effect of SRB Forward Attachment Design and Comparison to Fence BMOs}

Figures 43 through 58 present $\Delta C_{p, r m s}$ trends for the detailed forward attachment, simplified forward attachment, sharp SRB fence, blunt SRB fence, and core stage fence configurations for the SLS-10005 vehicle. Mach numbers of $0.90,0.95,1.10$, and 1.2 are presented in these figures for the core and both boosters, with the highest $\Delta C_{p, r m s}$ levels seen at transonic conditions at Mach 0.90 and 0.95. Buffet environments are less severe at supersonic Mach numbers, which is typical for launch vehicles. Peak $\Delta C_{p, r m s}$ values are seen in Figures 43, 47, 51, and 55 on the core at transducer azimuths of $90^{\circ}$ and $270^{\circ}$ which correspond to the regions facing the boosters and downstream of the booster forward attachment. These peak fluctuating pressures in the region downstream of the forward SRB attachments are quite high in magnitude when compared to other launch vehicle buffet environments. The simplified geometry of the 2012 test forward attachment protuberance can be seen to have the highest peak $\Delta C_{p, r m s}$. This peak is significantly reduced with the updated detailed booster forward attachment shape and the various fence configurations are shown to also reduce the peak $\Delta C_{p, r m s}$ further, with the booster fence configurations appearing to have the most significant impact on peak $\Delta C_{p, r m s}$. The "poke-out" in $\Delta C_{p, r m s}$ on the MPCV seen in Figure 43 for Mach 0.90 is due to the presence of a normal shock at that station which randomly shifts its longitudinal position. The buffet environments observed on the core at the 45/135/225/315 azimuths in Figures 44, 48, 52, and 56 are seen to possess a more benign degree of pressure fluctuations than at the $0^{\circ}$ and $270^{\circ}$ azimuths. Transducer measurements on the booster $45 / 135 / 225 / 315^{\circ}$ azimuths found in Figures 45, 46, 49, 50, 53, 54, 57, and 58 show higher environments on the core stage side of each booster and lower environments on the outboard sides of boosters.

\section{Comparison of SRB Nose Cone BMOs to the Baseline Detailed Forward Attachment Protuberance}

The buffet environments of the booster nose cone BMOs are compared to the detailed forward attachment protuberance in Figures 59 through 74 for the SLS-10005 vehicle configuration. These figures show that each of the three booster nose cone BMO geometries significantly reduce the peak environments, with the bent bi-conic nose cone providing slightly greater reductions in $\Delta C_{p, r m s}$ on the core. At the Mach 0.90 transonic condition, the canted straight SRB nose cones reduce the peak $\Delta C_{p, r m s}$ levels at the $90^{\circ}$ and $270^{\circ}$ azimuths seen in Figure 59, however, these nose cones are also seen to increase $\Delta C_{p, r m s}$ levels downstream of nose cones on the outboard sides of the SRBs as seen in Figures 61 and 62. The canted ogive SRB nose cone configuration is seen to result in higher $\Delta C_{p, r m s}$ levels at all azimuths at stations on the nose cone for the Mach 1.10 condition shown in Figures 69 and 70 . At the buffet-critical transonic conditions of Mach 0.90 and 0.95 , the bent bi-conic SRB nose cone provides slightly more reduction in peak $\Delta C_{p, r m s}$ levels on the core stage behind the SRB forward attachment at azimuths $90^{\circ}$ and $270^{\circ}$ as shown in Figures 59 and 63.

\section{Conclusion}

The test development and results of the Space Launch System (SLS) buffet environments program at the LaRC Transonic Dynamics Tunnel (TDT) have been presented in detail including preliminary comparisons of buffet environments. Model development, instrumentation design, and data acquisition system strategies were discussed to illustrate methods utilized to ensure the buffet environments were measured with an eye for data quality. Buffet environments obtained during the 2012 buffet test for the crewed SLS-10003 and SLS-13000 configurations and the SLS-11000 shrouded payload configuration have been presented and show very high localized buffet $\Delta C_{p, r m s}$ 
downstream of the booster forward attachment protuberance in regions where the core and booster face each other. In response to high buffet environments observed during initial testing in 2012, increased geometric fidelity of booster forward attachment protuberances and six buffet mitigation options (BMOs) were tested during the 2014 TDT test entry. Test data results of $\Delta C_{p, r m s}$ presented in this paper have shown that updated protuberance geometry and the $\mathrm{BMO}$ nose cones and fences reduce the buffet environments observed in regions downstream of the booster forward attachment on core and booster. These BMOs provide design points for the SLS program to choose from if it is determined that reducing buffet loads further should be pursued in future structural design analysis cycles.

\section{References}

1. Jones, George W., Foughner, Jerome T., Jr., "Investigation of Buffet Pressures on Models of Large Manned Launch Vehicle Configurations.” NASA TN-D-1663, May 1963.

2. Rainey, Gerald A., "Progress on the Launch-Vehicle Buffeting Problem.” Journal of Spacecraft and Rockets, Volume 2, Number 3, May-June 1965.

3. Cole, Henry A., Jr.; Erickson, A. L.; and Rainey, A. G.: "Buffeting During Atmospheric Ascent.” NASA SP8001, 1963, Revised November 1970.

4. Fleming, E.R., "Transonic Buffeting Loads Experience at The Aerospace Corporation.” The Aerospace Corporation Report No. TOR-95(5530)-6. March 1995.

5. Schutzenhofer, Luke A., Howard, Paul W.: “Acquisition and Reduction of Large Volumes of Fluctuating Data.” Aerophysics Research at MSFC: Research Achievements Review. Volume III, Report No. 4. NASA TM X53799, 1968.

6. Piatak, D.J.; Sekula, M.K.; and Rausch, R.D.: "Ares Launch Vehicle Transonic Buffet Testing and Analysis Techniques.” Journal of Spacecraft and Rockets. Vol. 49. No 5. September-October 2012.

7. Sekula, M.K.; Piatak, D.J.,; Rausch, R.D.: “Analysis of Crew Launch Vehicle Transonic Alternating Flow Phenomenon.” Journal of Spacecraft and Rockets. Vol. 49. No. 5. September-October 2012.

8. Kabe, A.M., “Time Domain Buffet Loads Analysis.” The Aerospace Corporation Report Number TOR2007(2209)-6078. November 17, 2006.

9. Piatak, D.J.; Sekula, M.K.; and Rausch, R.D.: "Comparison of Ares I-X Wind-Tunnel-Derived Buffet Environment with Flight Data.” Journal of Spacecraft and Rockets. Vol. 49. No 5. September-October 2012.

10. Cole, Stanley R.; Keller, Donald F.; and Piatak, David J.: "Contributions of the NASA Langley Transonic Dynamics Tunnel to Launch Vehicle and Spacecraft Development.” AIAA 2000-1772, Presented at the AIAA Dynamics Specialists Conference, Atlanta, GA, April 5-6, 2000.

11. Cole, Stanley R., Garcia, Jerry L.: "Past, Present, and Future Capabilities of the Transonic Dynamics Tunnel from an Aeroelasticity Perspective.” AIAA 2000-1767, Presented at the AIAA Dynamics Specialists Conference, Atlanta, GA, April 506, 2000.

12. Cole, Stanley R.; Johnson, R. Keith; Piatak, David J.; Florance, Jennifer P.; and Rivera, José A., Jr.: “Test Activities in the Transonic Dynamics Tunnel and a Summary of Recent Facility Improvements.” AIAA 20031958, Presented at the AIAA Dynamics Specialists Conference, Norfolk, VA, April 9-10, 2003.

13. Blevins, Robert D., “Flow Induced Vibration.” Van Nostrand Reinhold Publishers, ISBN 0442206518, 1990.

14. Crawford, Bradley L.: "Angle Measurement System (AMS) for Establishing Model Pitch and Roll Zero, and Performing Single Axis Angle Comparisons.” AIAA Paper 2007-1162, 45th AIAA Aerospace Sciences Meeting and Exhibit, Reno, NV, 8-11 Jan. 2007. 
15. Sekula, M.K., Piatak, D.J., Rausch, R.D., Florance, J.R., and Ramey, J.M.: "Initial Assessment of Space Launch System Transonic Unsteady Pressure Environment.” 53 ${ }^{\text {rd }}$ AIAA Aerospace Sciences Meeting, Kissimmee, FL, 2015.

Table 1. Space Launch System RBM TDT test section conditions in R134a test medium.

\begin{tabular}{|c|c|c|c|c|c|}
\hline $\begin{array}{c}\text { Mach } \\
\text { Number }\end{array}$ & $\mathbf{q}, \mathbf{p s f}$ & $\begin{array}{c}\mathbf{V}, \\
\mathbf{f t} / \mathbf{s}\end{array}$ & $\mathbf{R e}, / \mathbf{f t}$ & $\begin{array}{c}\text { Re, 1st } \\
\text { Stage Dia. }\end{array}$ & $\begin{array}{c}\text { Model Scale } \\
\text { Buffet } \\
\text { Bandwidth, Hz } \\
\text { (Aeroacoustic) }\end{array}$ \\
\hline \hline 0.8 & 481 & 441 & $8.47 \mathrm{E}+06$ & $7.05 \mathrm{E}+06$ & $\begin{array}{c}0-1,002 \\
(0-33,390)\end{array}$ \\
\hline 0.9 & 480 & 499 & $7.47 \mathrm{E}+06$ & $6.22 \mathrm{E}+06$ & $\begin{array}{c}0-1,002 \\
(0-33,388)\end{array}$ \\
\hline 1.0 & 480 & 546 & $6.92 \mathrm{E}+06$ & $5.76 \mathrm{E}+06$ & $\begin{array}{c}0-1,004 \\
(0-33,482)\end{array}$ \\
\hline 1.2 & 300 & 653 & $3.66 \mathrm{E}+06$ & $3.05 \mathrm{E}+06$ & $\begin{array}{c}0-1,059 \\
(0-35,301)\end{array}$ \\
\hline
\end{tabular}

Table 2. Rigid buffet model scaling laws.

\begin{tabular}{|c|c|}
\hline $\begin{array}{c}\text { Quantity } \\
\text { to be } \\
\text { Scaled }\end{array}$ & Full scale to Model scale Relationship \\
\hline \hline Pressure & $p_{f s}=p_{m s} \frac{q_{f s}}{q_{m s}}$ \\
\hline Force & $F_{f s}=F_{m s} \frac{q_{f s}}{q_{m s}}\left(\frac{D_{f s}}{D_{m s}}\right)^{2}$ \\
\hline Time & $T_{f s}=T_{m s} \frac{D_{f s}}{D_{m s}} \frac{V_{m s}}{V_{f s}}$ \\
\hline Frequency & $f_{f s}=f_{m s} \frac{D_{m s}}{D_{f s}} \frac{V_{f s}}{V_{m s}}$ \\
\hline
\end{tabular}

Table 3. SLS buffet model Kulite unsteady pressure transducers.

\begin{tabular}{|c|c|c|c|}
\hline Kulite Model & $\begin{array}{c}\text { Measurement } \\
\text { Type }\end{array}$ & $\begin{array}{c}\text { Pressure } \\
\text { Range, psi } \\
\text { (differential) }\end{array}$ & Bandwidth \\
\hline \hline XCL-17-IA-100-15D & Buffet & $+/-15$ & $3 \mathrm{KHz}+/-0.5 \mathrm{~dB}$ \\
\hline XCL-30-IA-072-15D & Buffet & $+/-15$ & $3 \mathrm{KHz}+/-0.5 \mathrm{~dB}$ \\
\hline XCL-31-IA-072-15D & Acoustic & $+/-15$ & $57 \mathrm{KHz}+/-0.5 \mathrm{~dB}$ \\
\hline
\end{tabular}

Table 4. Installation geometry for Kulite pressure transducers.

\begin{tabular}{|c|c|c|c|c|c|}
\hline $\begin{array}{c}\text { Installation } \\
\text { Type }\end{array}$ & $\begin{array}{c}\text { Dorifice, }_{\text {in }} \\
\text { in }\end{array}$ & $\begin{array}{c}\text { D }_{\text {sensor, }} \\
\text { in }\end{array}$ & $\begin{array}{c}\text { Lorifice, } \\
\text { in }\end{array}$ & $\begin{array}{c}\text { Lsensor, } \\
\text { in }\end{array}$ & $\begin{array}{c}\text { Cavity } \\
\text { Volume, in } \\
\text { (frustum only) }\end{array}$ \\
\hline \hline 100 Buffet & 0.040 & 0.105 & 0.032 & 0.052 & $8.8095 \mathrm{e}-05$ \\
\hline 072 Buffet & 0.040 & 0.081 & 0.040 & 0.052 & $3.5817 \mathrm{e}-5$ \\
\hline 072 Acoustic & 0.040 & 0.081 & 0.010 & n/a & 0 \\
\hline
\end{tabular}


Table 5. Cavity resonance parameters and predictions for RBM Kulites.

\begin{tabular}{|c|c|c|c|c|}
\hline $\begin{array}{c}\text { Installation } \\
\text { Type }\end{array}$ & A $_{\text {orifice, }}$ in $^{\mathbf{2}}$ & $\begin{array}{c}\mathbf{V}_{\text {cavity, }} \text { in }^{\mathbf{3}} \\
\text { [frustum }+ \\
\text { sensor] }\end{array}$ & $\begin{array}{c}\text { Lorifice, } \\
\text { in }\end{array}$ & $\begin{array}{c}\text { Predicted } \\
\text { Cavity } \\
\text { Resonance, } \\
\text { KHz (R134a) }\end{array}$ \\
\hline 093 Buffet & $1.25664 \mathrm{e}-3$ & $1.4310 \mathrm{e}-04$ & 0.032 & 15.93 \\
\hline 072 Buffet & $1.25664 \mathrm{e}-3$ & $9.0817 \mathrm{e}-005$ & 0.040 & 18.59 \\
\hline 072 Acoustic & $1.25664 \mathrm{e}-3$ & $1.4000 \mathrm{e}-005$ & 0.010 & 78.31 \\
\hline
\end{tabular}

Table 6. Instrumentation and Data Acquisition Summary.

\begin{tabular}{|c|c|c|c|c|c|}
\hline & $\begin{array}{c}\text { OA-DAS } \\
\text { NEFF 620 } \\
500 \mathrm{~Hz} \text { scan } \\
200 \mathrm{~Hz} \text { filter } \\
\end{array}$ & $\begin{array}{c}\text { MIIDAS } \\
\text { NEFF } 730 \\
12 \mathrm{KHz} \text { scan } \\
4.5 \mathrm{KHz} \text { filter } \\
\end{array}$ & $\begin{array}{c}\text { DSPCon } \\
\text { Piranha III } \\
100 \mathrm{KHz} \text { scan } \\
50 \mathrm{KHz} \text { filter } \\
\end{array}$ & 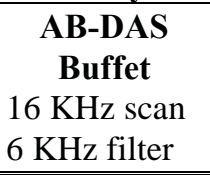 & $\begin{array}{c}\text { AB-DAS } \\
\text { Aeroacoustic } \\
200 \mathrm{KHz} \text { scan } \\
60 \mathrm{KHz} \text { filter } \\
\end{array}$ \\
\hline \multicolumn{6}{|l|}{2012 Test } \\
\hline Tunnel Parameters & All params. & & & & \\
\hline $\begin{array}{c}\text { Tri-axial } \\
\text { Model Orientation } \\
\text { Q-Flex }\end{array}$ & 3-channels & & & & \\
\hline $\begin{array}{c}\text { Sting AOA } \\
\text { Q-Flex }\end{array}$ & 1-channel & & & & \\
\hline Accelerometers & 4-channels & & & & \\
\hline $\begin{array}{l}\text { ESP Steady } \\
\text { Pressure }\end{array}$ & 64-pressures & & & & \\
\hline Buffet Kulites & & 360-channels & & & \\
\hline $\begin{array}{c}\text { Aeroacoustic } \\
\text { Kulites } \\
\end{array}$ & & & 64-channels & & \\
\hline \multicolumn{6}{|l|}{2014 Test } \\
\hline Tunnel Parameters & All params. & & & All params. & \\
\hline $\begin{array}{c}\text { Tri-axial } \\
\text { Model Orientation } \\
\text { Q-Flex }\end{array}$ & 3-channels & & & 3-channels & \\
\hline $\begin{array}{c}\text { Sting AOA } \\
\text { Q-Flex }\end{array}$ & 1-channel & & & 1-channel & \\
\hline Accelerometers & 4-channels & & & 4-channels & \\
\hline $\begin{array}{l}\text { ESP Steady } \\
\text { Pressure }\end{array}$ & $\begin{array}{l}\text { for cal. of } \\
\text { Kulites only }\end{array}$ & & & & \\
\hline Buffet Kulites & & & & 472-channels & \\
\hline $\begin{array}{c}\text { Aeroacoustic } \\
\text { Kulites }\end{array}$ & & & & & 32-channels \\
\hline
\end{tabular}



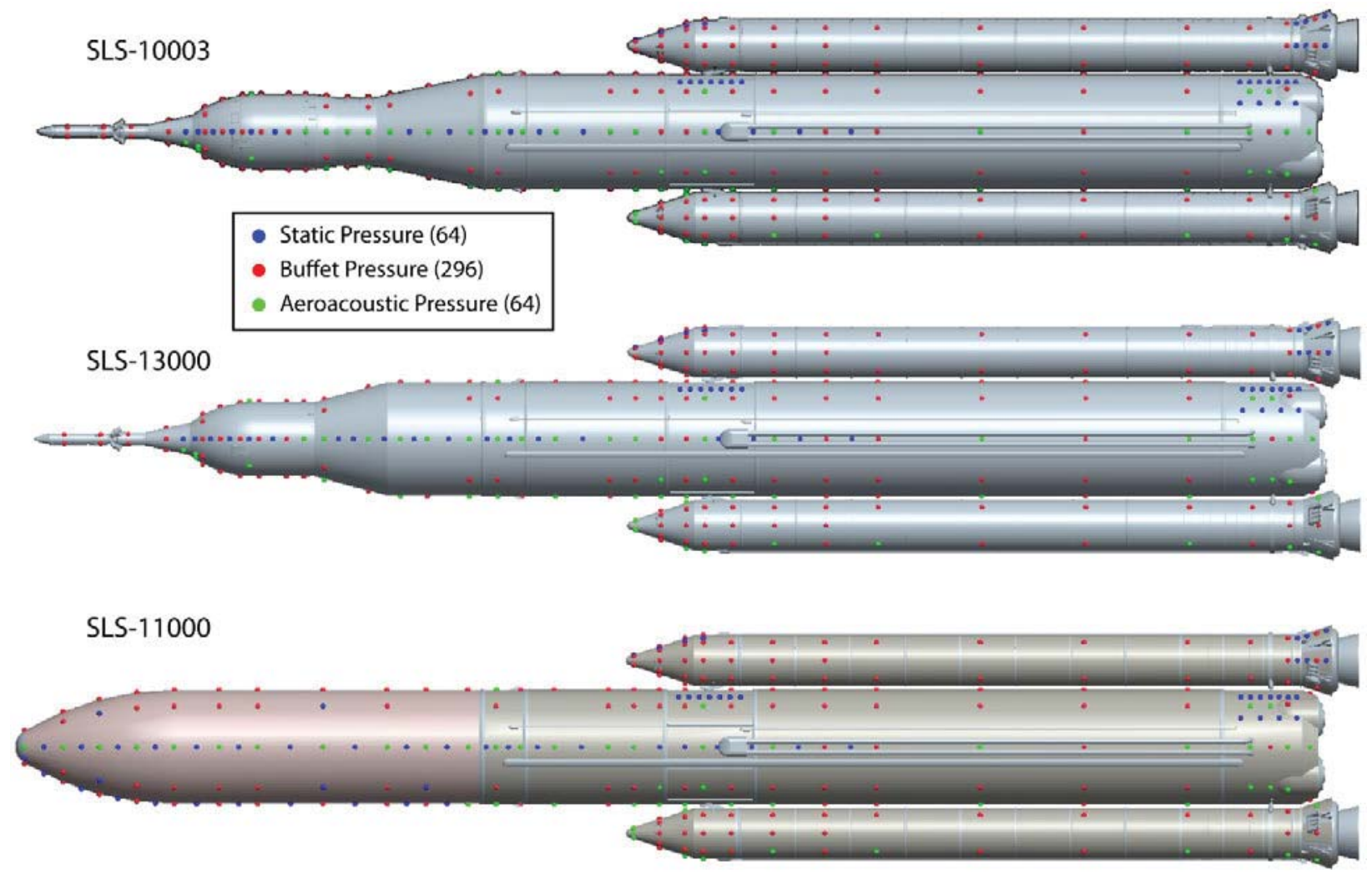

Figure 1. Space Launch System configurations tested during 2012 test at TDT.

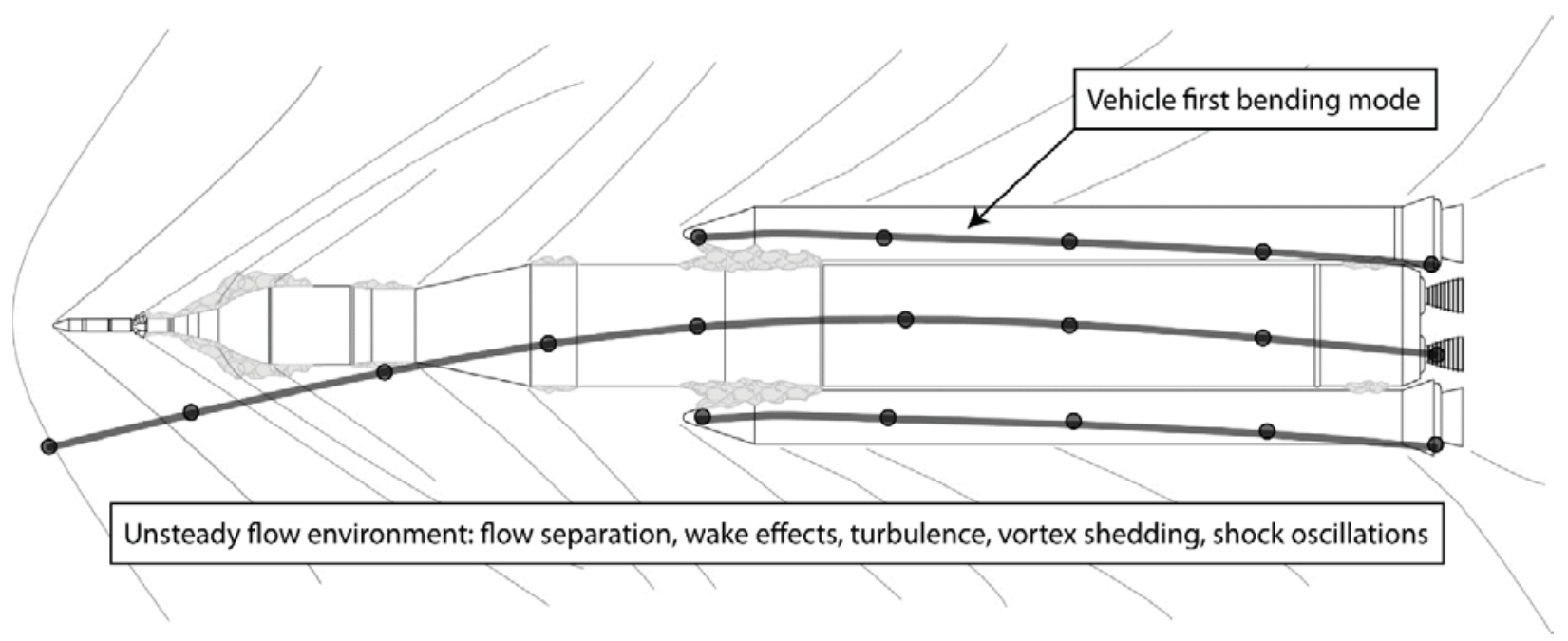

Figure 2. Notional unsteady flow environment and structural deflection for SLS configuration. 


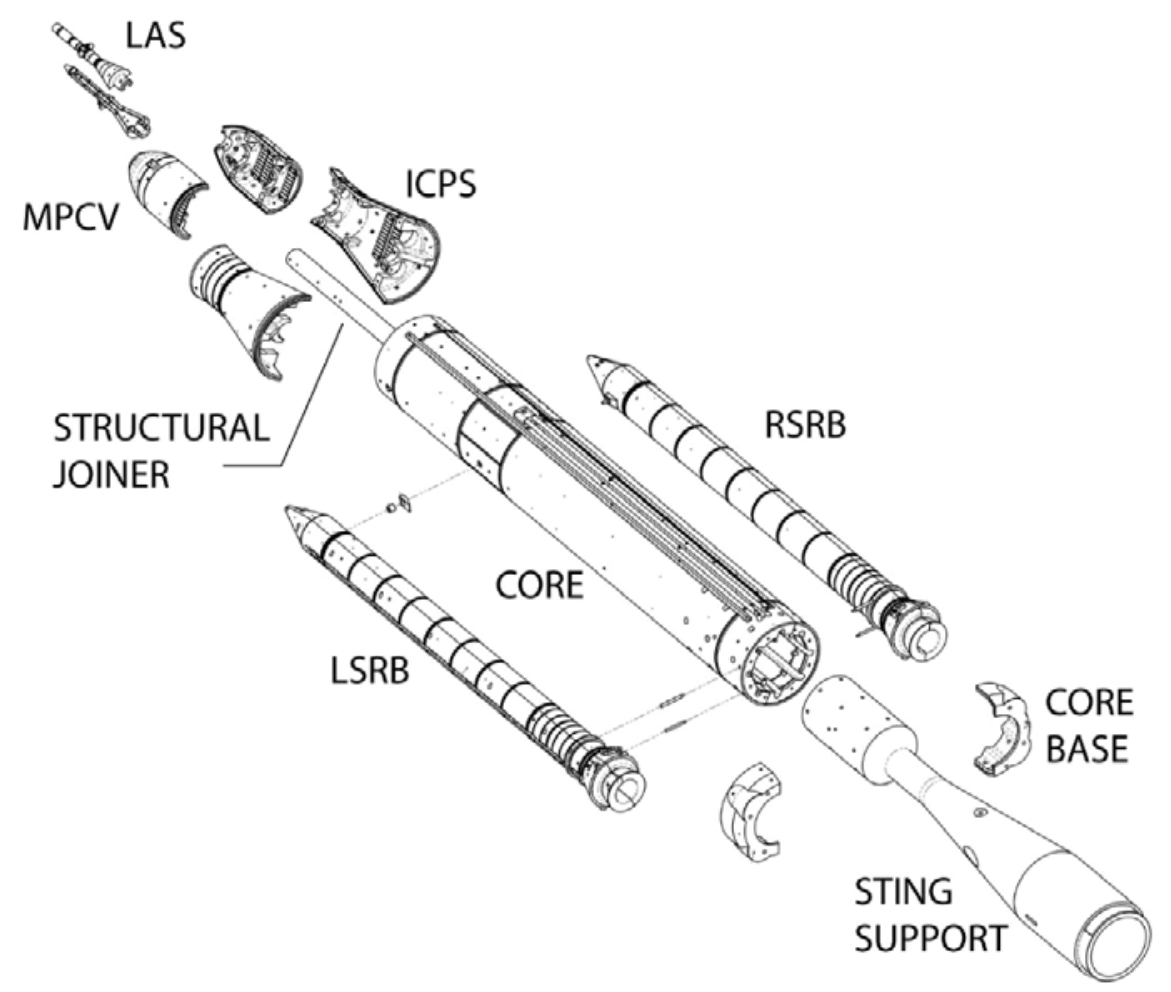

Figure 3. SLS rigid buffet model assembly components. (2012 test configuration shown)

\section{Forward Booster Attachment Protuberance}

\section{Test: Simplified}

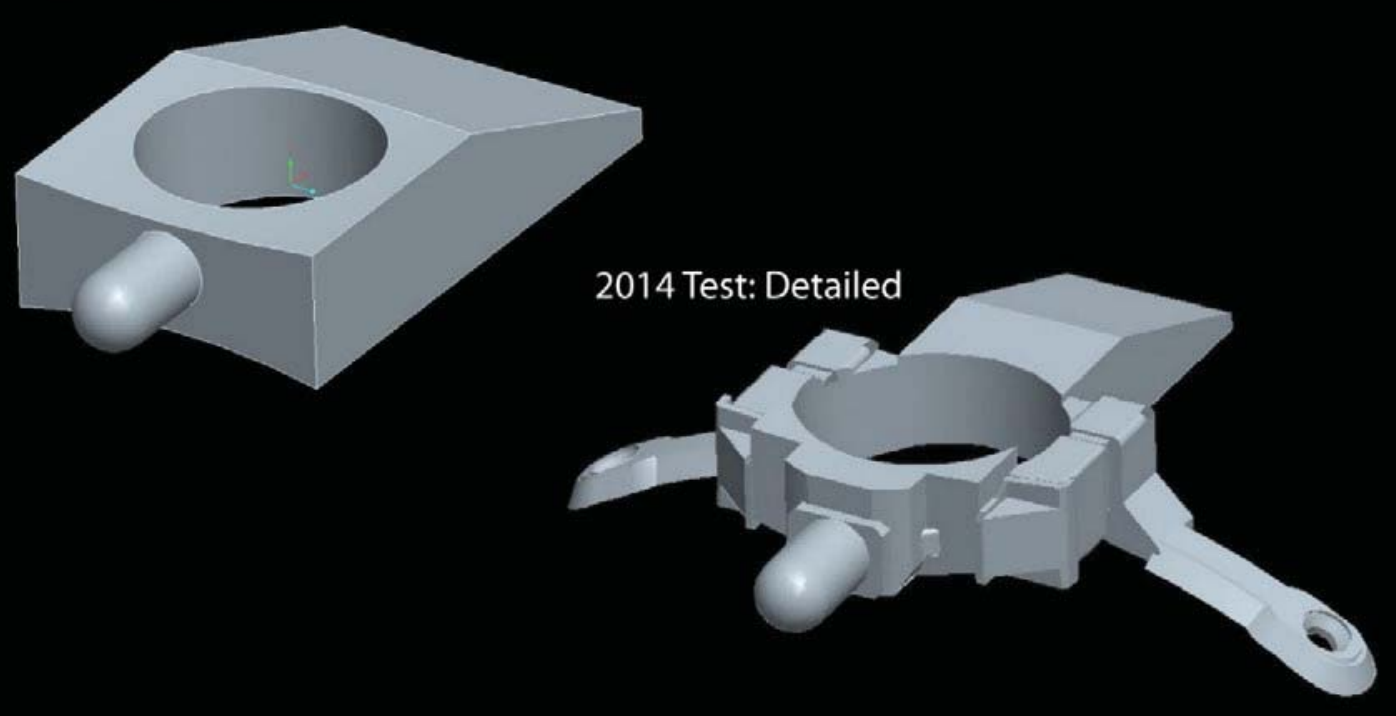

Figure 4. Comparison of simplified and detailed booster forward attachment protuberance. 


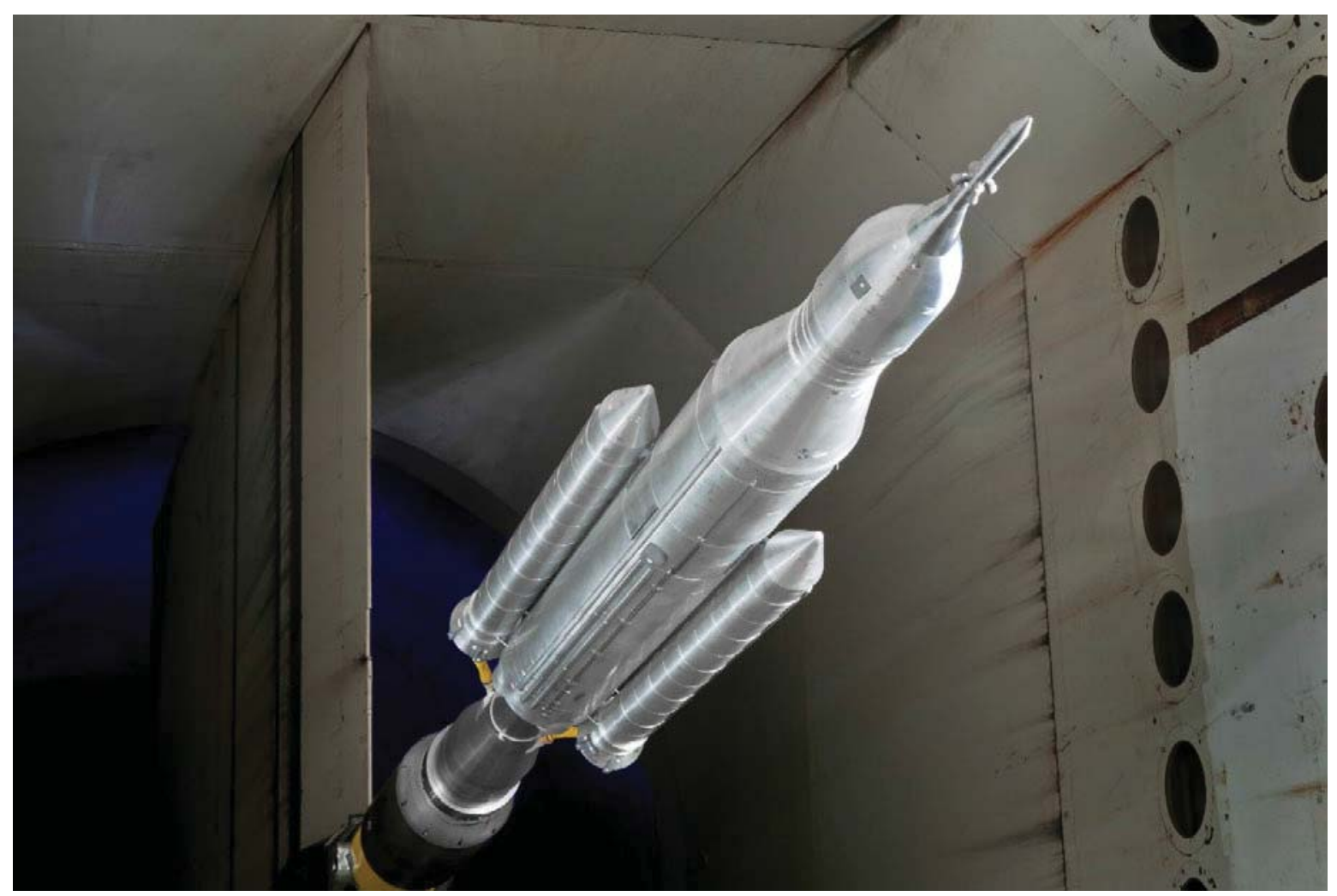

Figure 5. SLS-10003 rigid buffet model configuration. (2012 test)

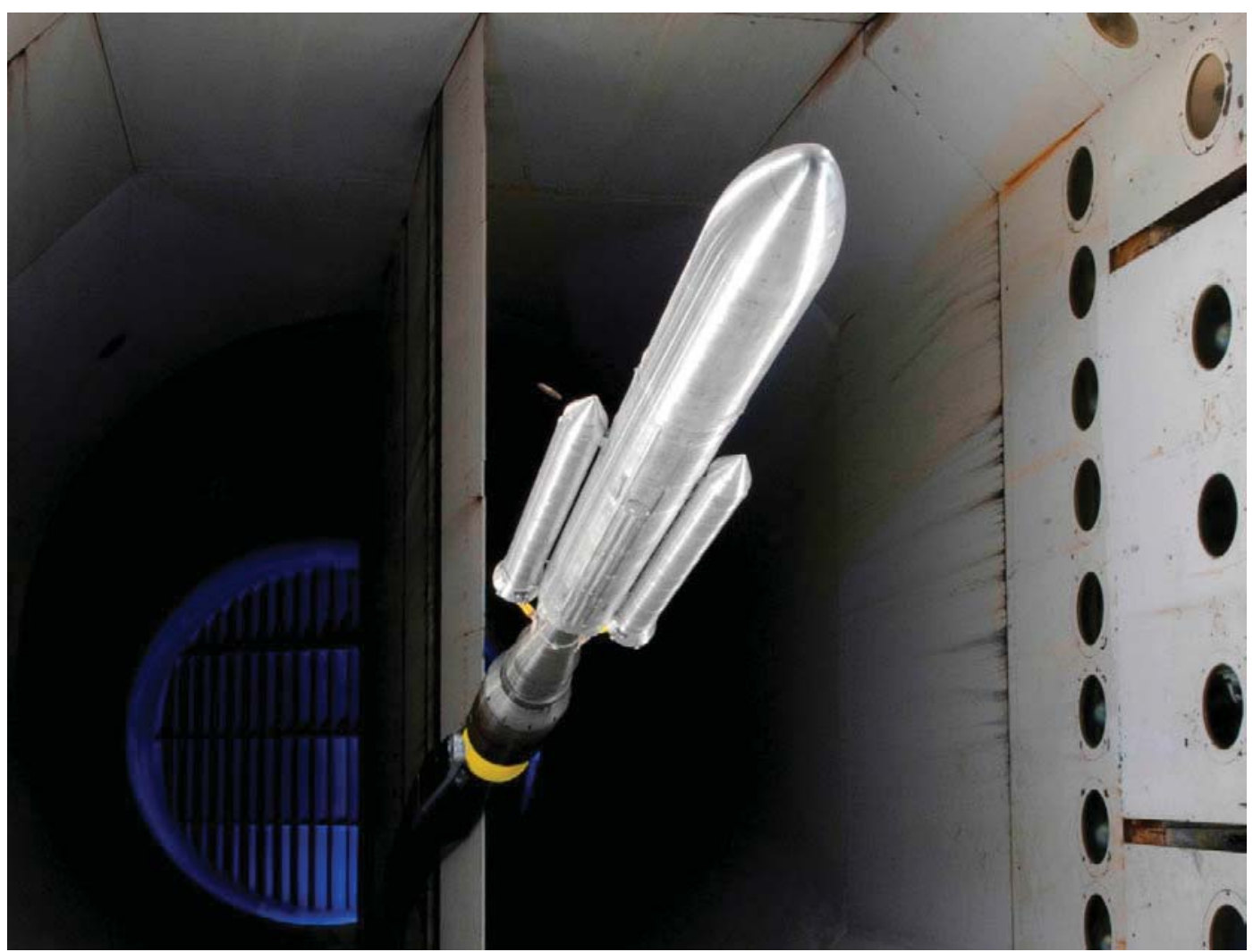

Figure 6. SLS-11000 rigid buffet model configuration. (2012 test) 


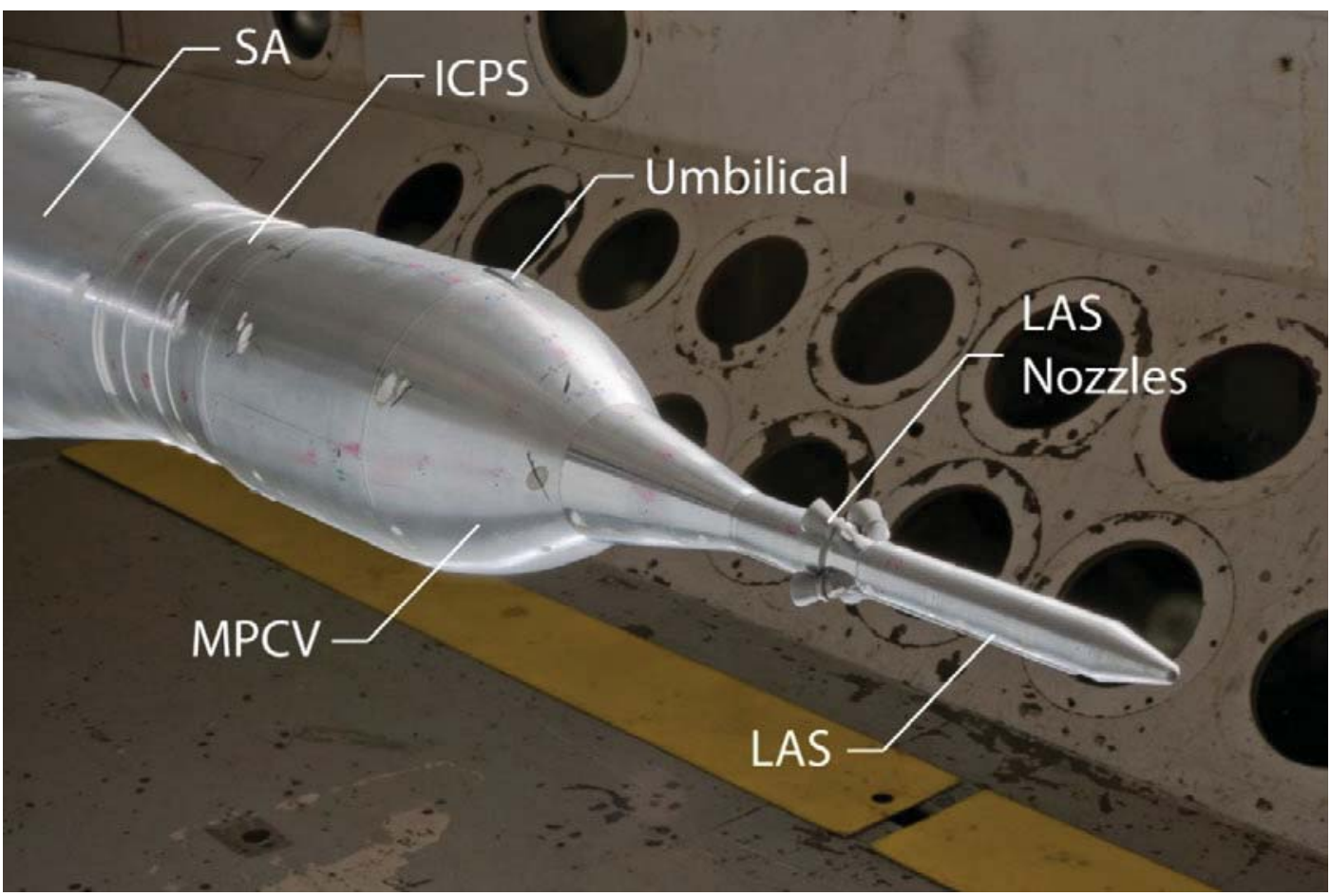

Figure 7. SLS rigid buffet model forward vehicle details. (2012 test)

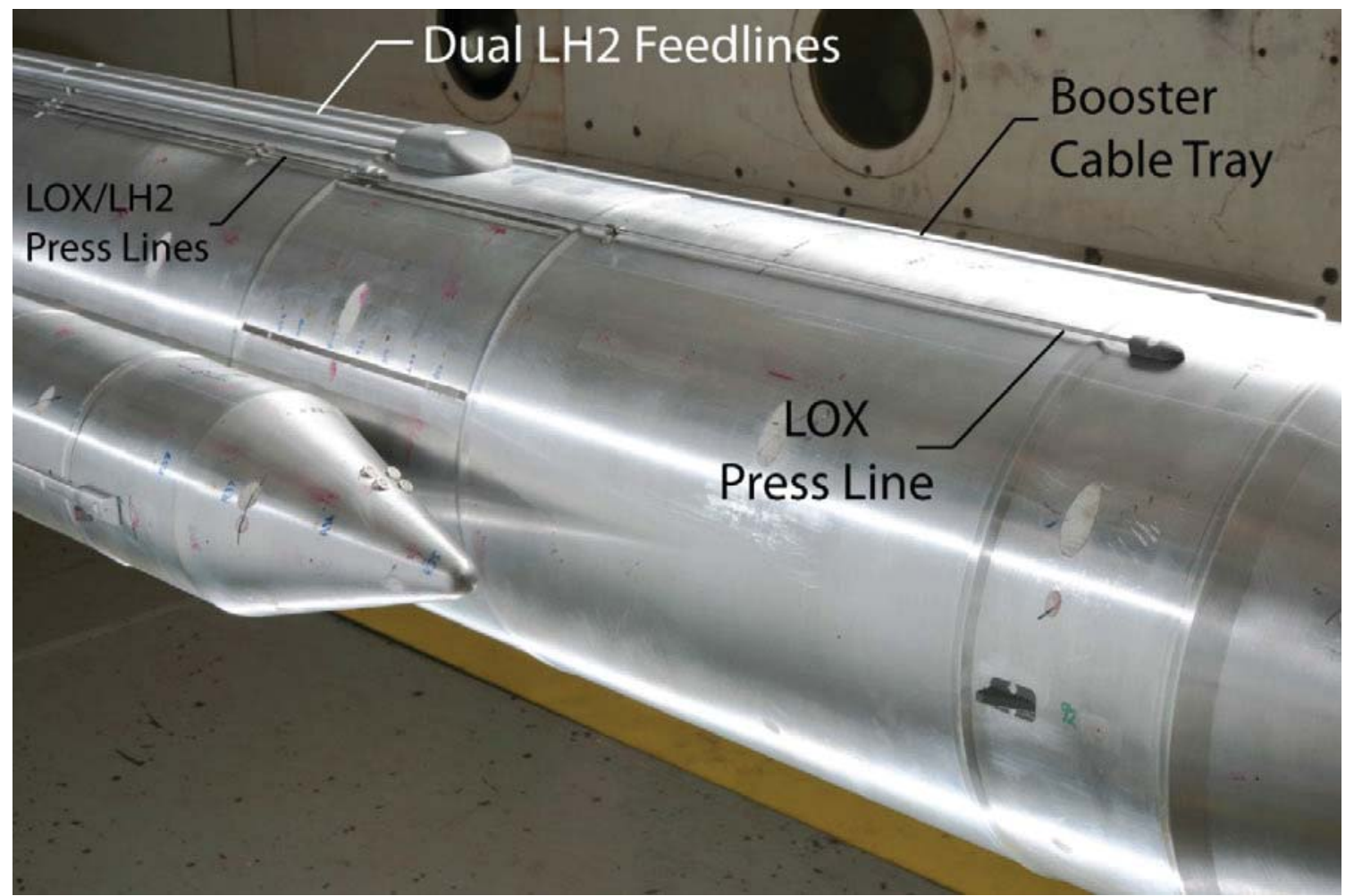

Figure 8. SLS rigid buffet model mid vehicle details. (2012 test) 


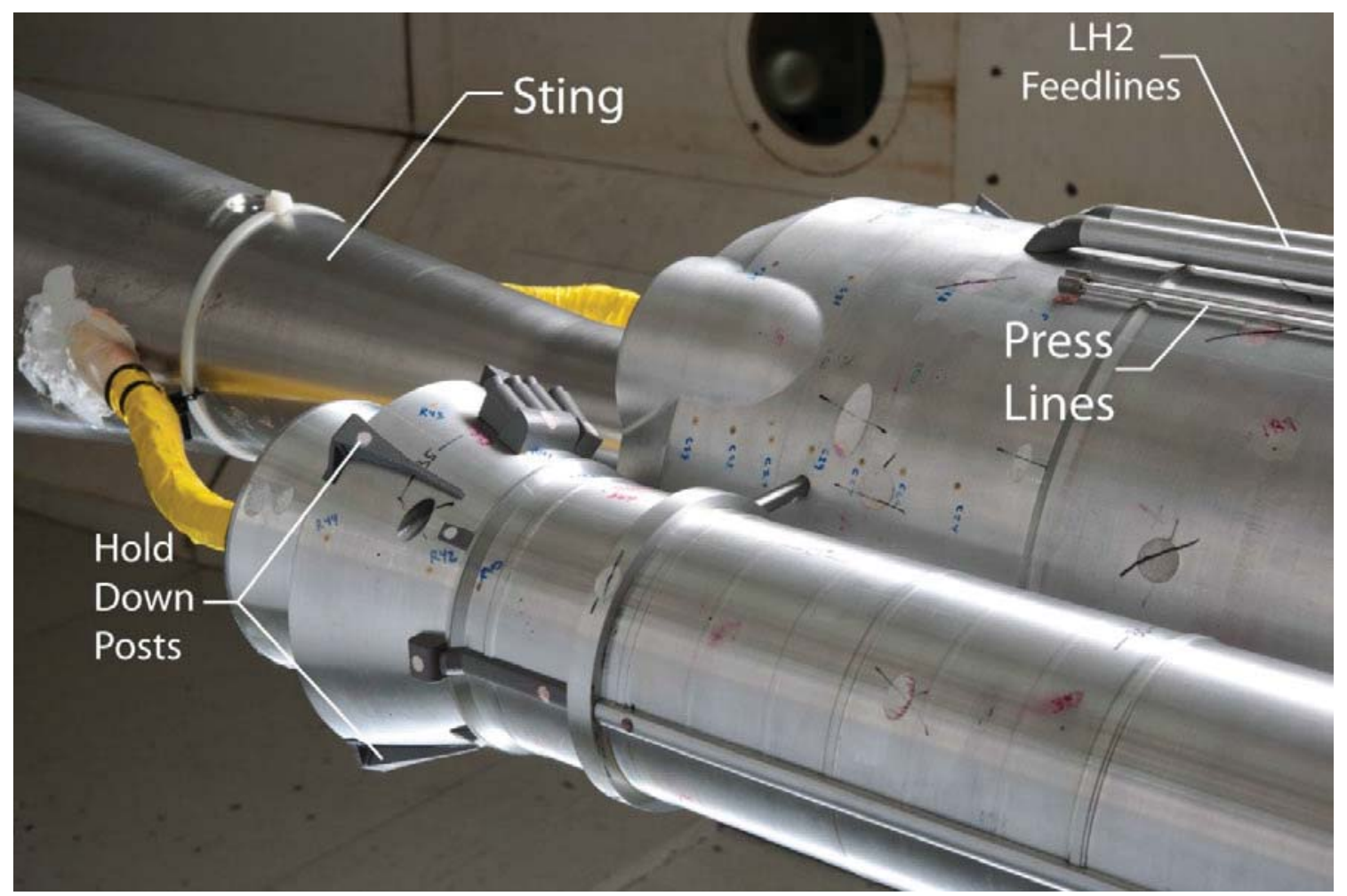

Figure 9. SLS rigid buffet model aft vehicle details. (2012 test)

- Buffet Pressure (296)

Station with additional azimuthal orifices

- Aeroacoustic Pressure (64)

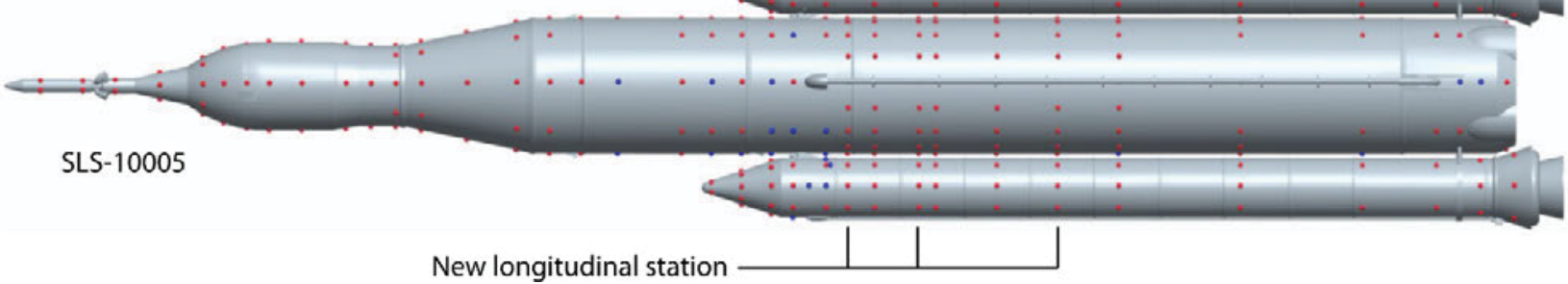

Figure 10. SLS transducer station locations/modifications. 


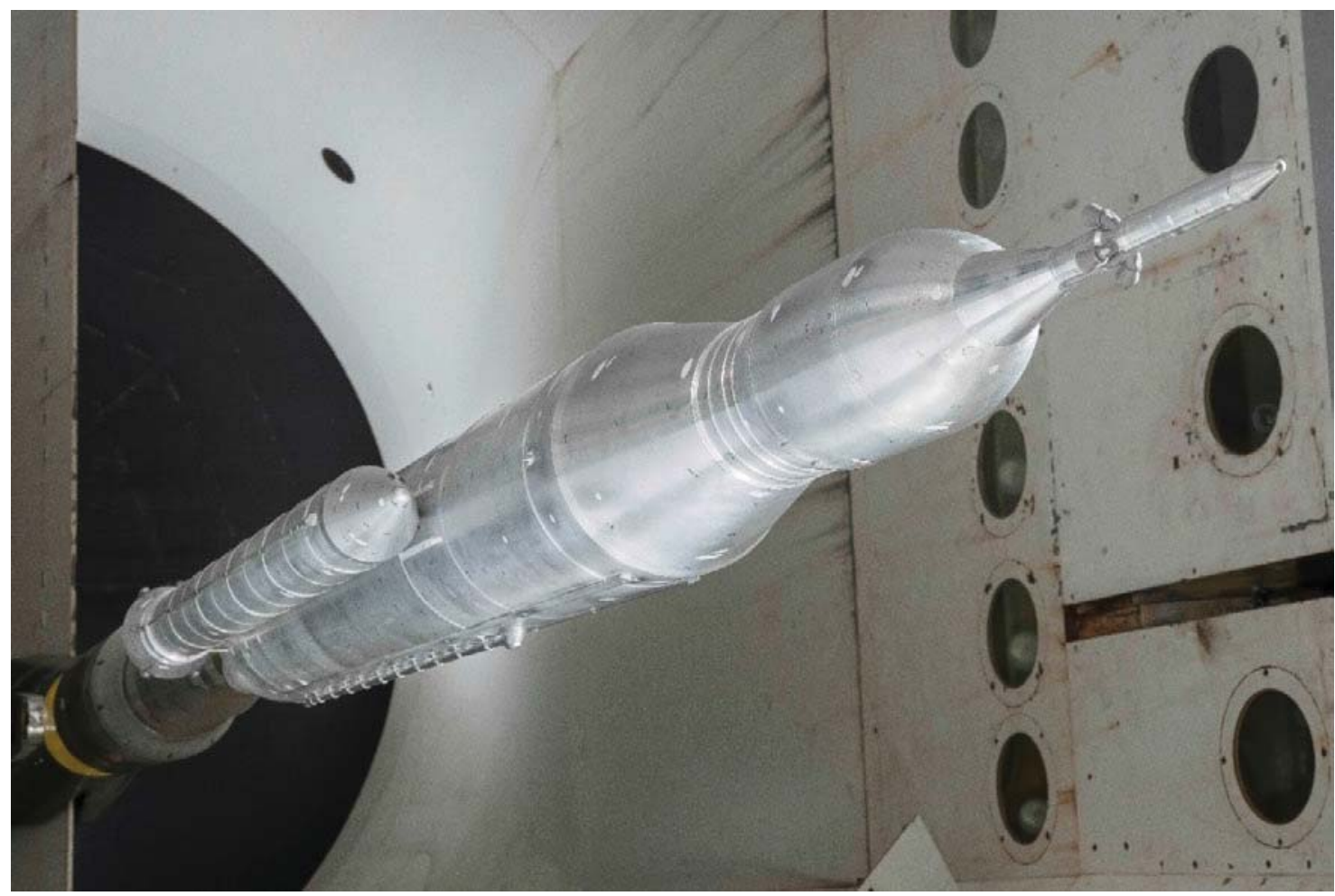

Figure 11. SLS-10005 rigid buffet model installed at TDT. (2014 test)

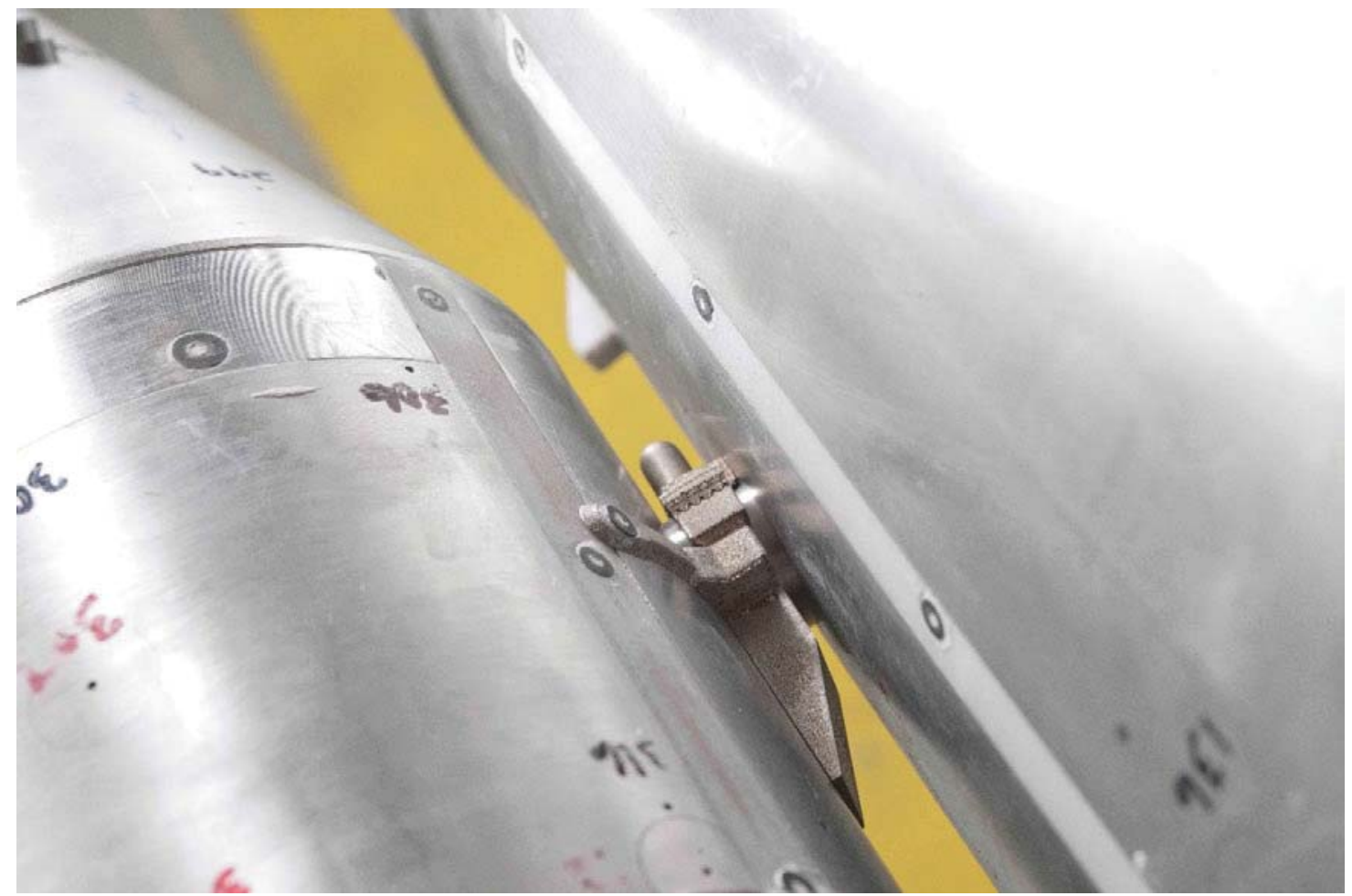

Figure 12. Detailed booster forward attachment protuberance. (2014 test) 


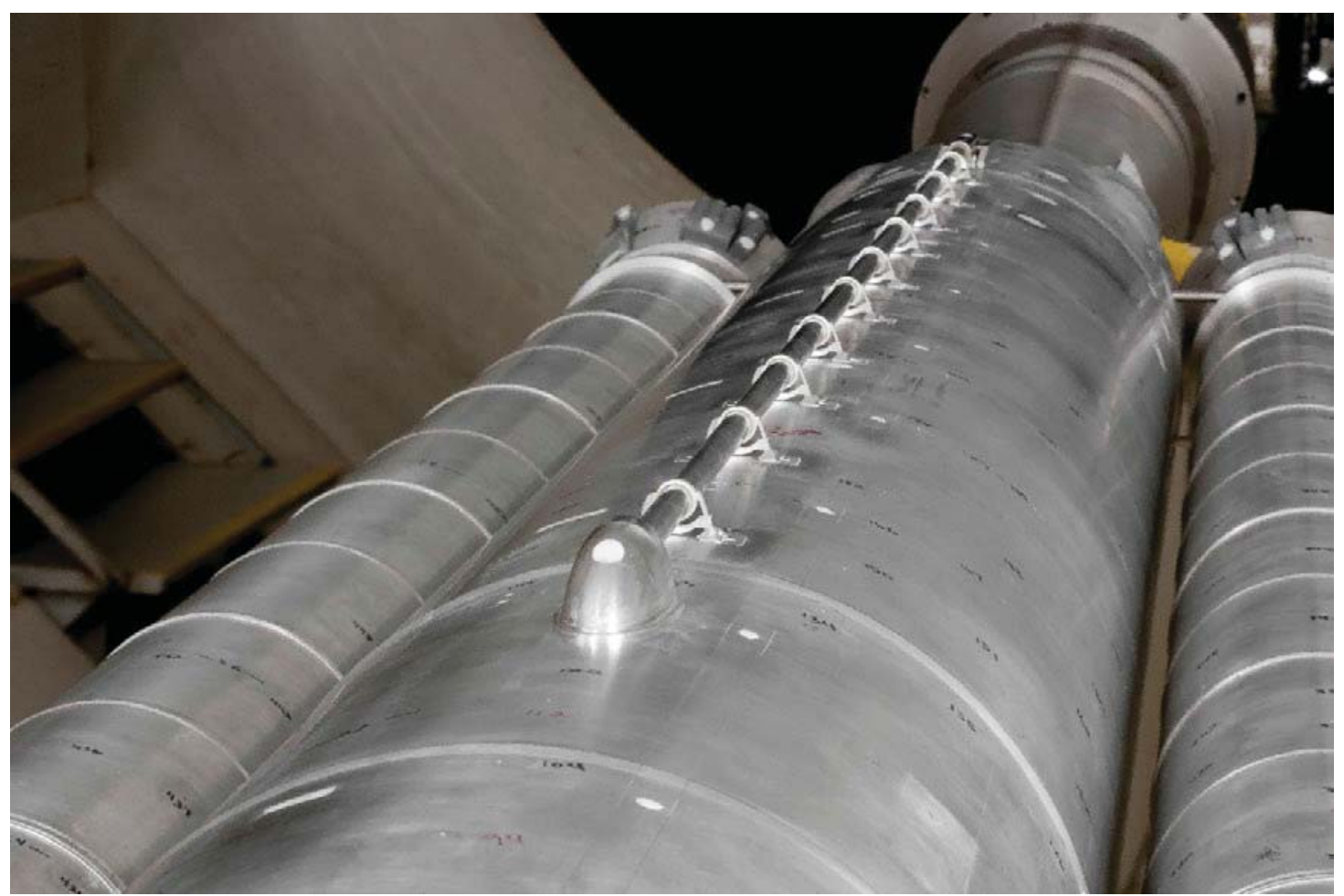

Figure 13. SLS-10005 LOX feedline details at 0 degree azimuth (top) of model. (2014 test)

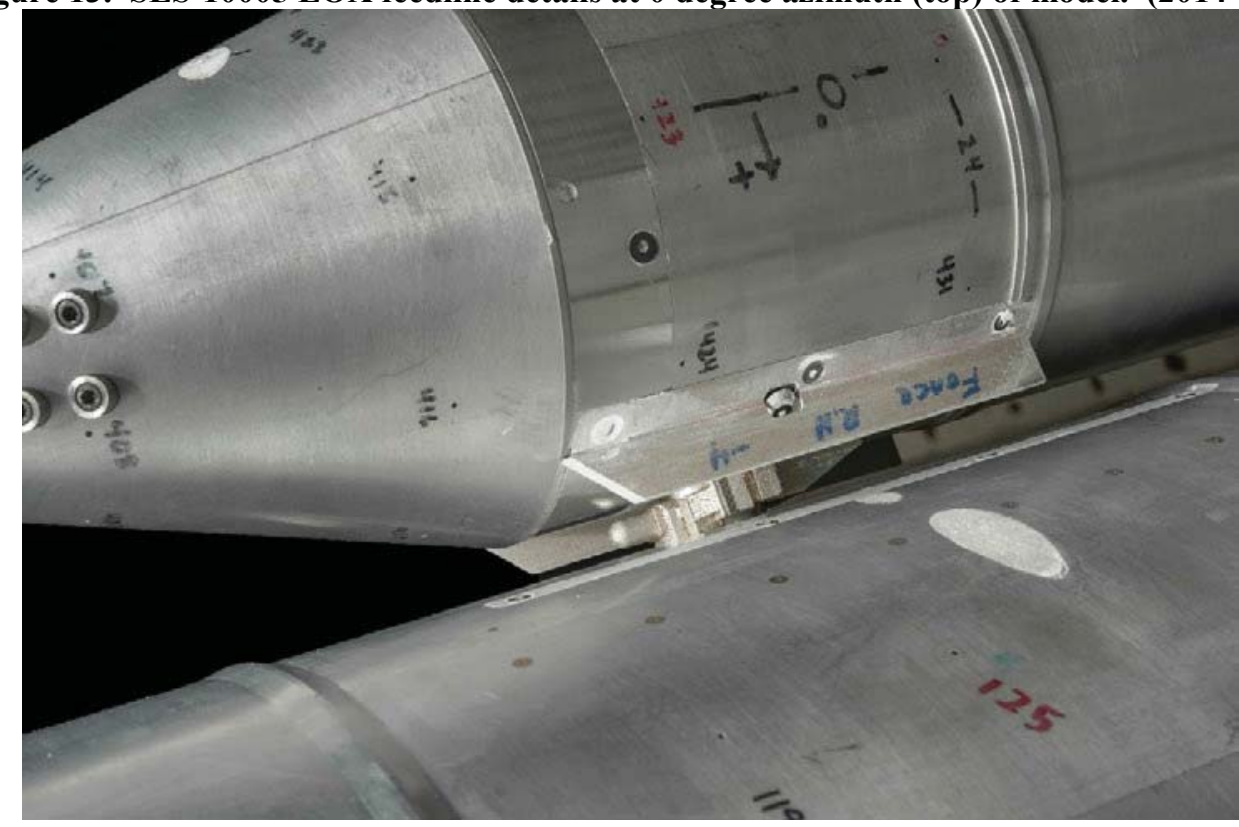

Figure 14. Sharp booster fences. (2014 test) 


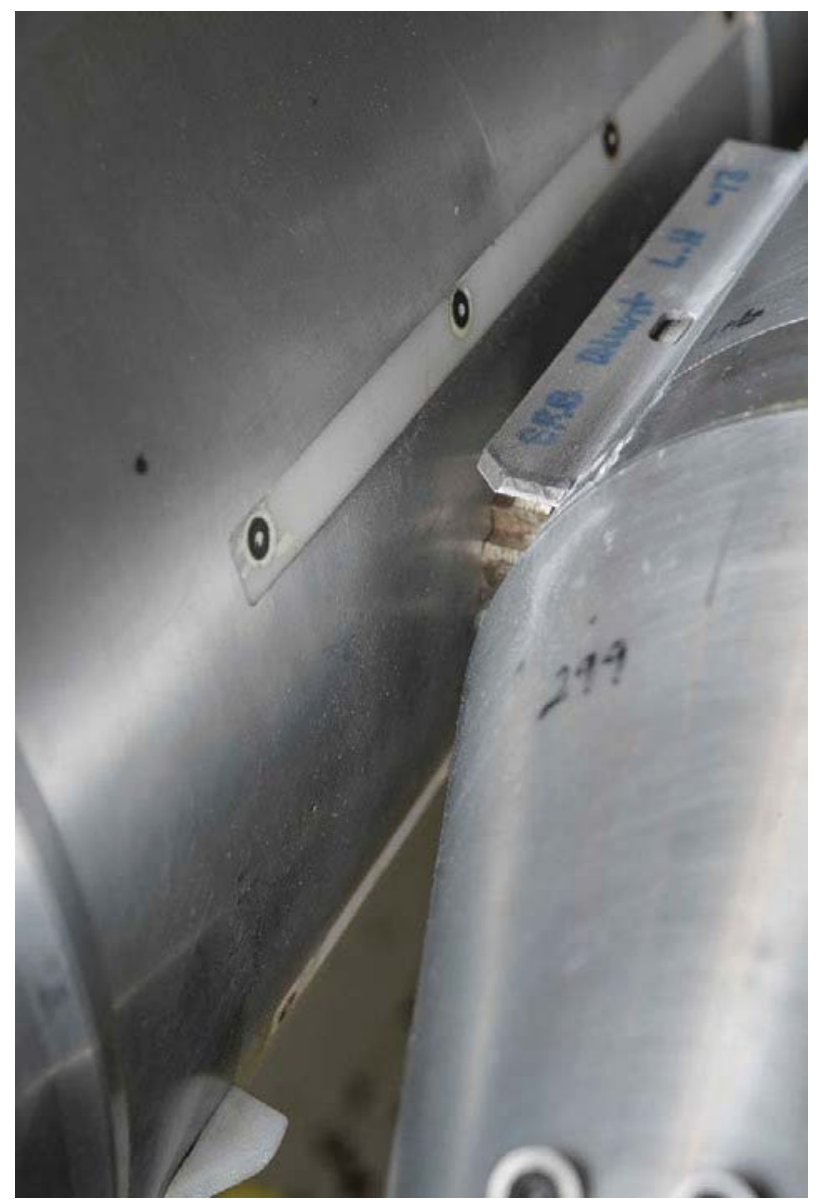

Figure 15. Blunt booster fence (lower fence obscured). (2014 test) 


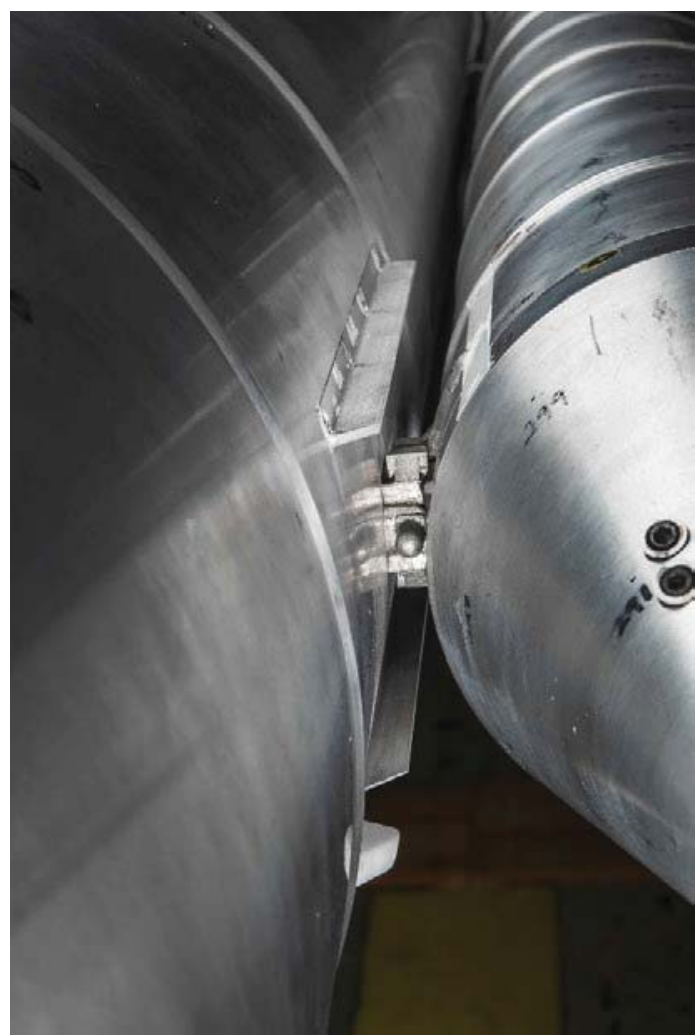

Figure 16. Core fences. (2014 test)

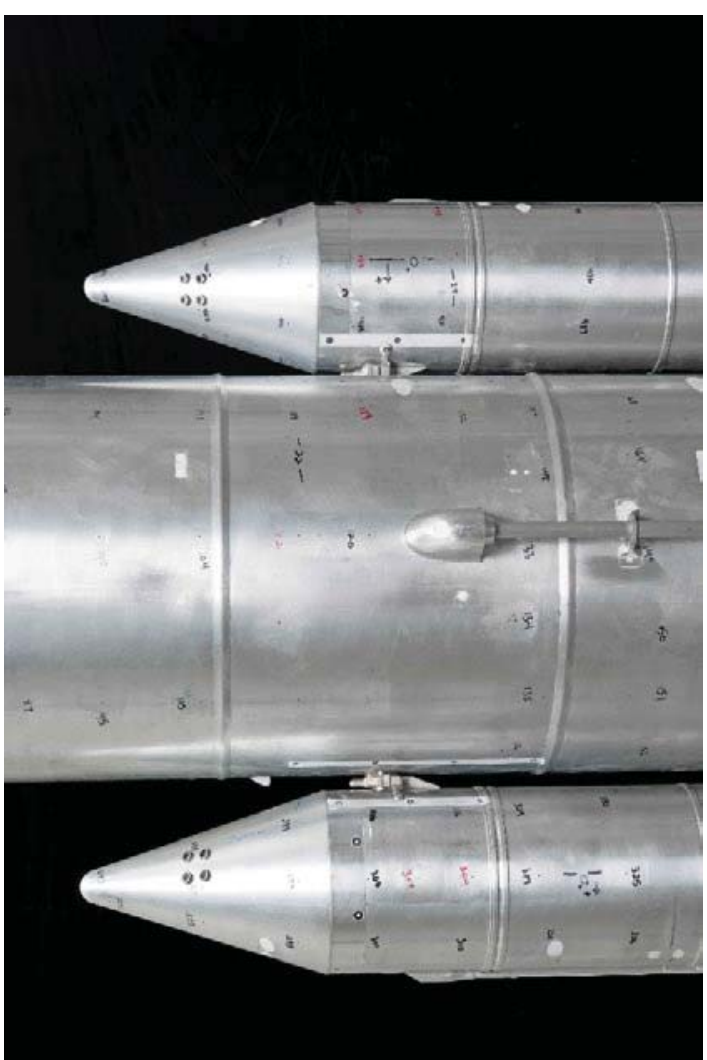

Figure 17. Baseline booster nose cones as viewed from top of model at 0 deg azimuth. (2014 test) 


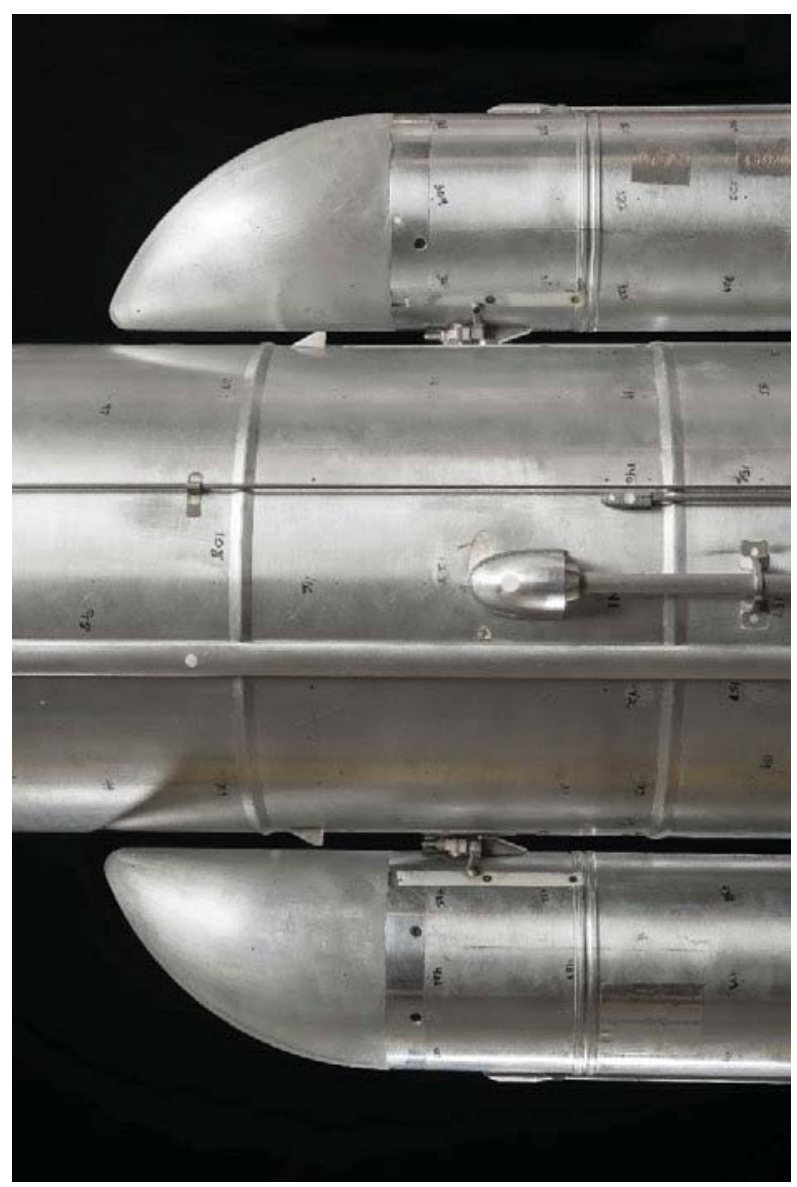

Figure 18. Canted ogive booster nose cones as viewed from bottom of model at 180 deg azimuth. (2014 test)

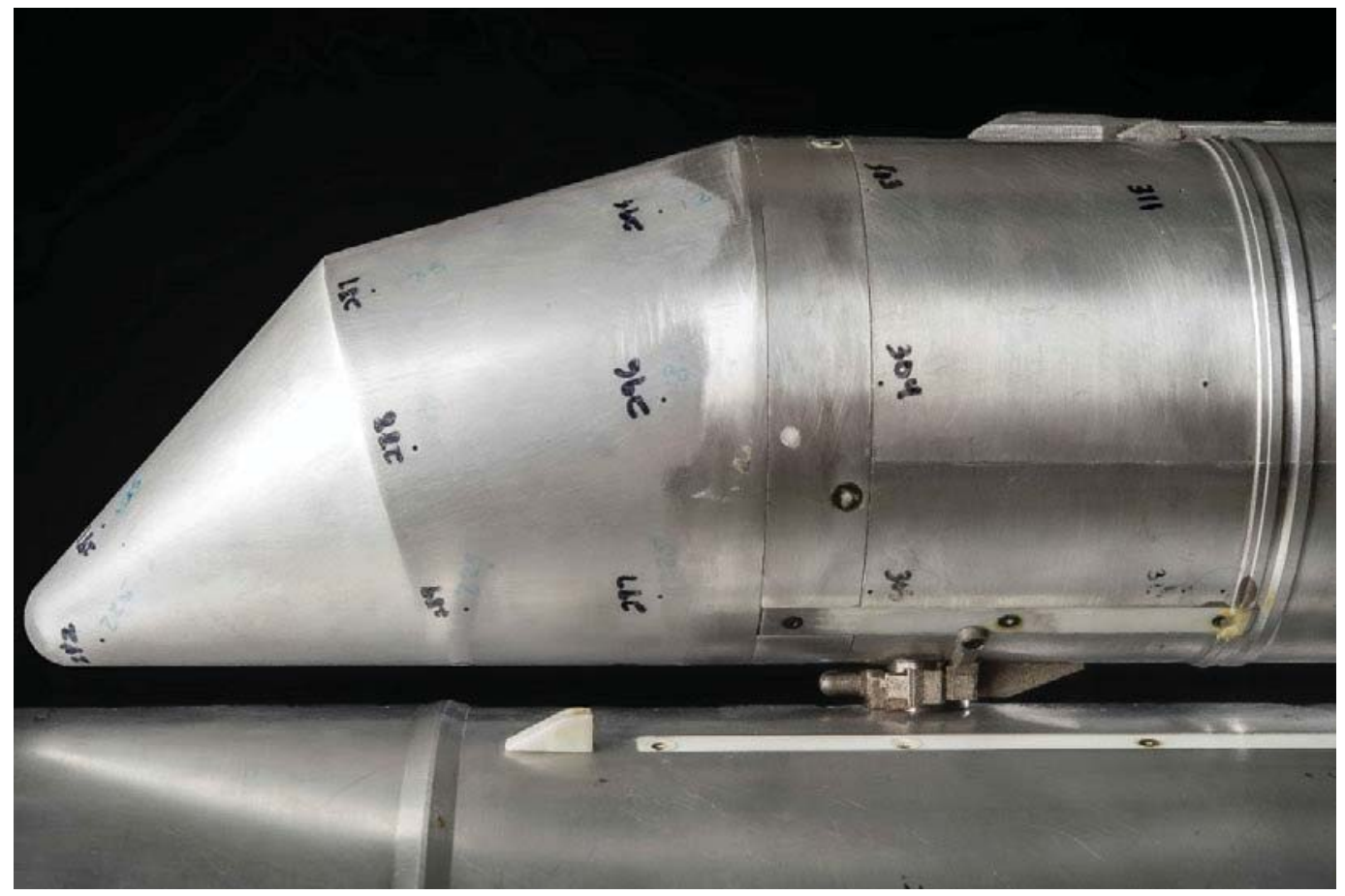

Figure 19. Bent bi-conic nose cone. (2014 test) 


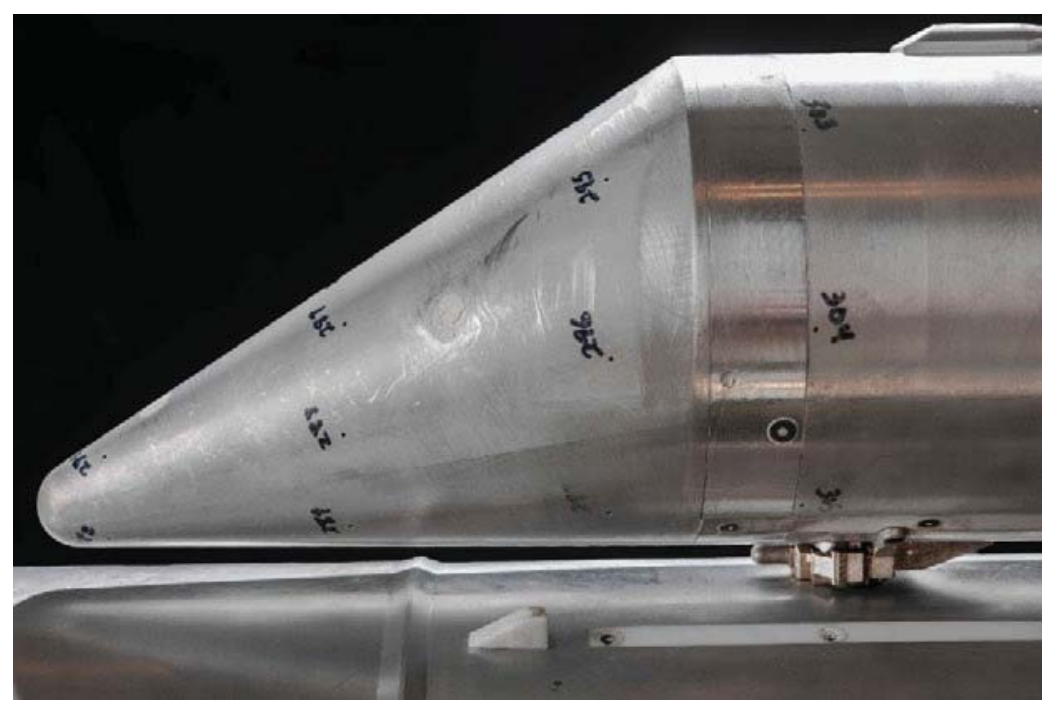

Figure 20. Canted straight nose cone. (2014 test)

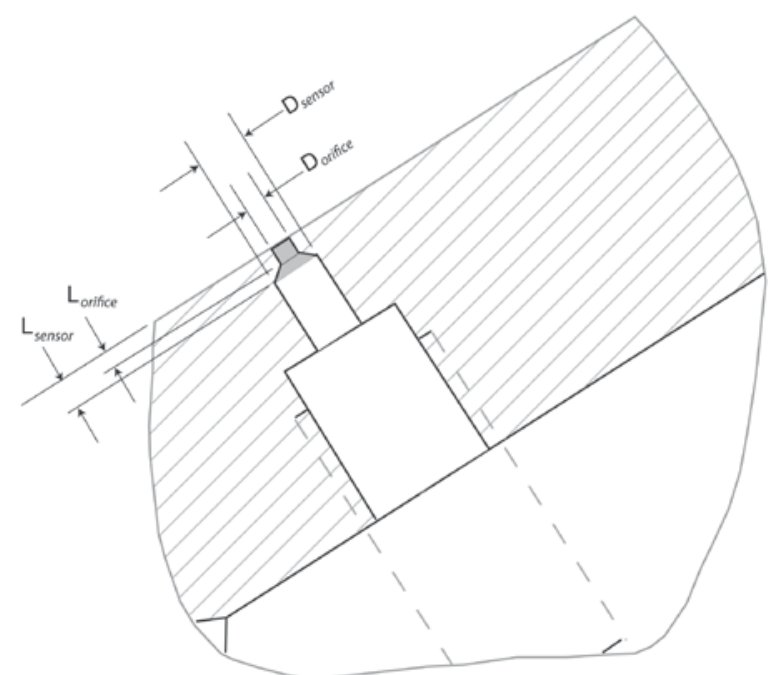

Figure 21. Installation geometry of buffet bandwidth Kulite unsteady pressure transducers.

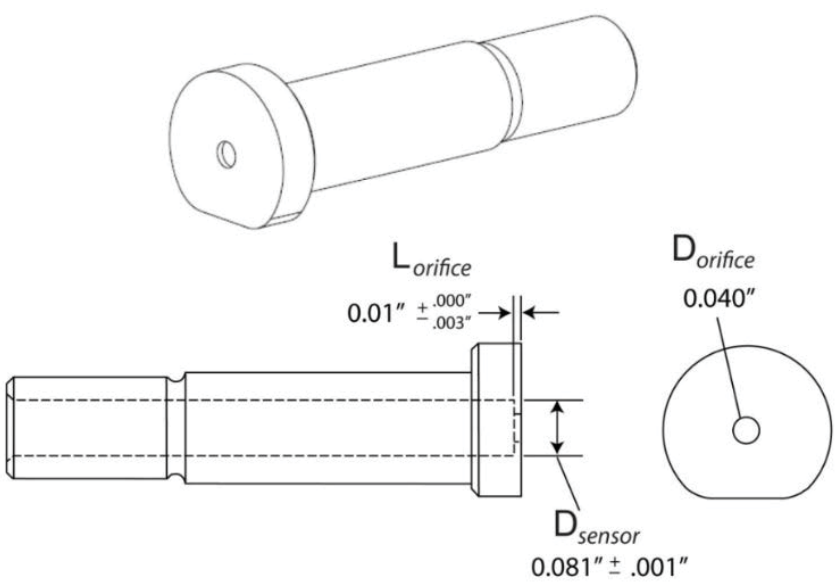

Figure 22. Aeroacoustic insert geometry. 


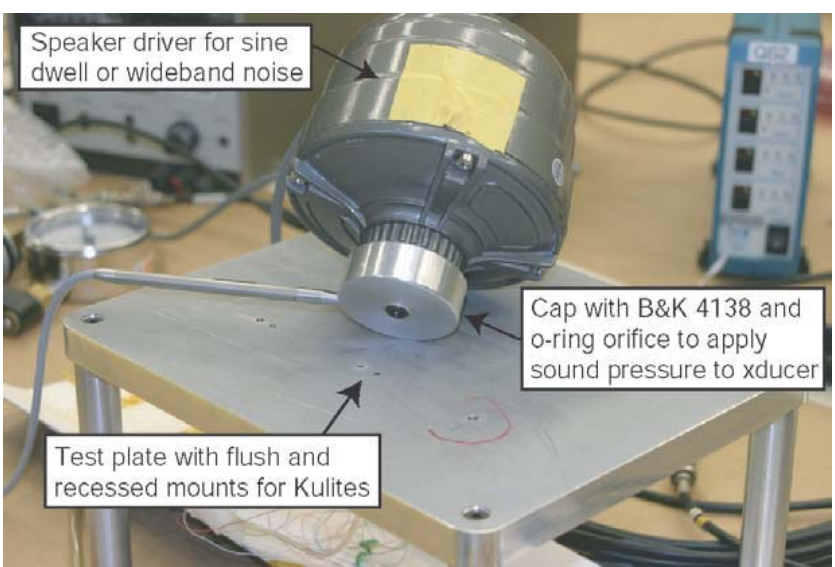

Figure 23. Speaker driver with acoustic test cap for pressure sensor frequency response measurement.

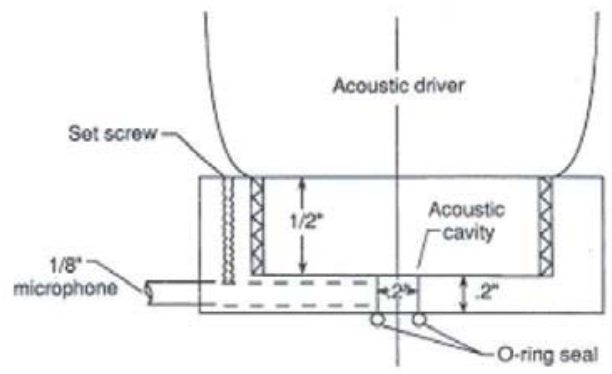

Figure 24. Details of acoustic test cap.
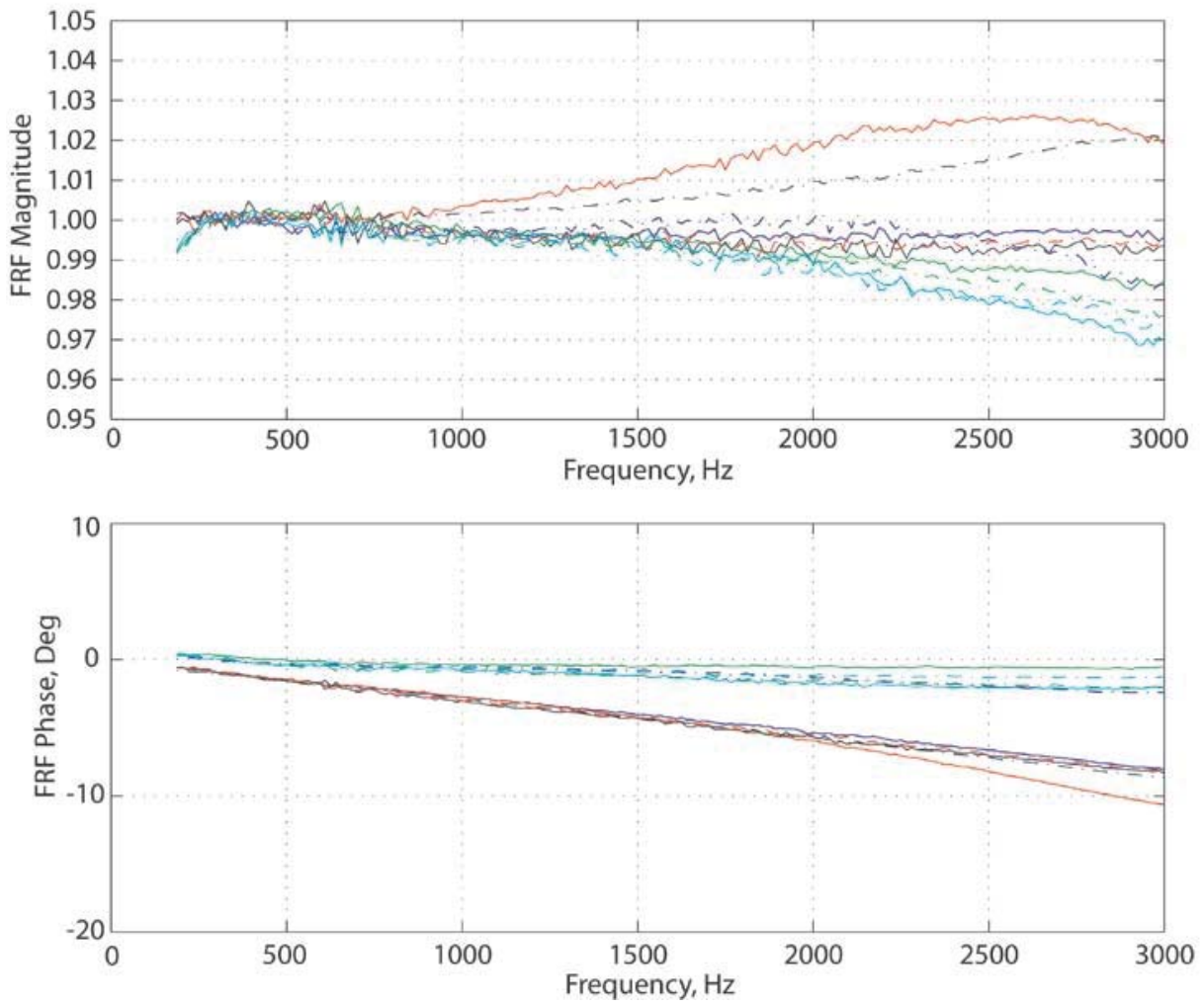

Figure 25. In-situ frequency response functions (FRFs) of representative SLS RBM buffet and aeroacoustic Kulite pressure transducers.

American Institute of Aeronautics and Astronautics 

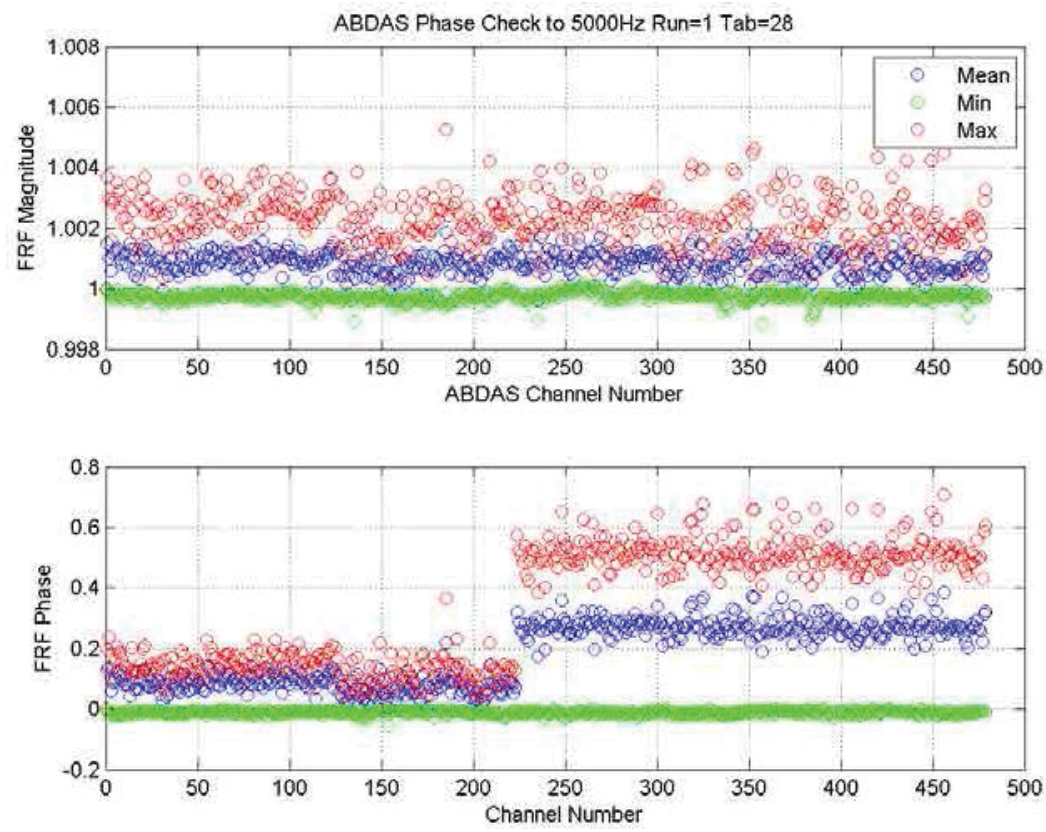

Figure 26. Channel-to-channel frequency response functions acquired using banded white noise applied to the ABDAS Precision Filter front-end calibration buss.

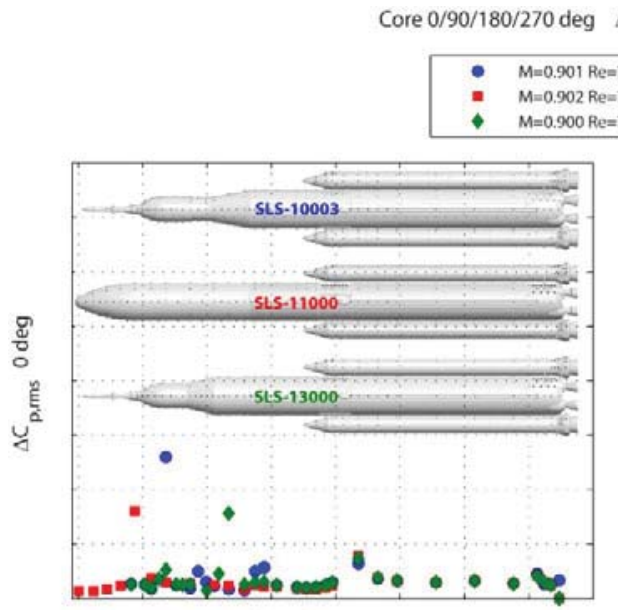

$\Delta C_{p, r m s}$ Bandpass $0.5-60 \mathrm{~Hz}$

Full Scale Station, in

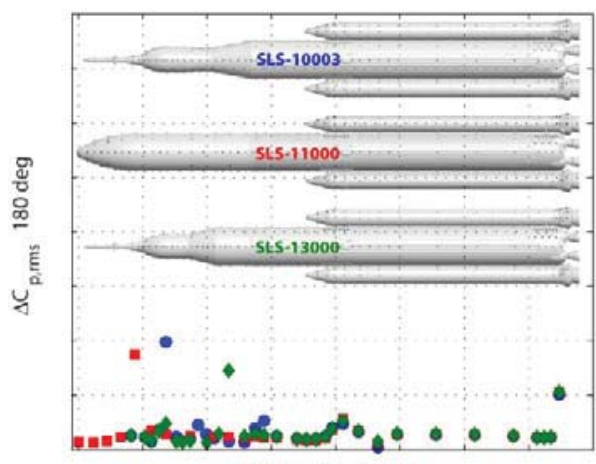

Full Scale Station, in

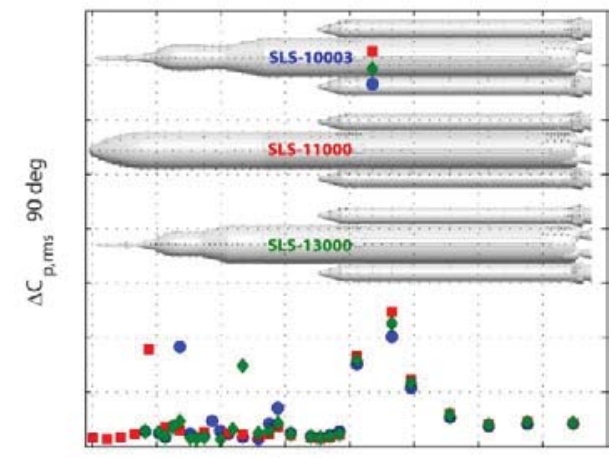

Full Scale Station, in

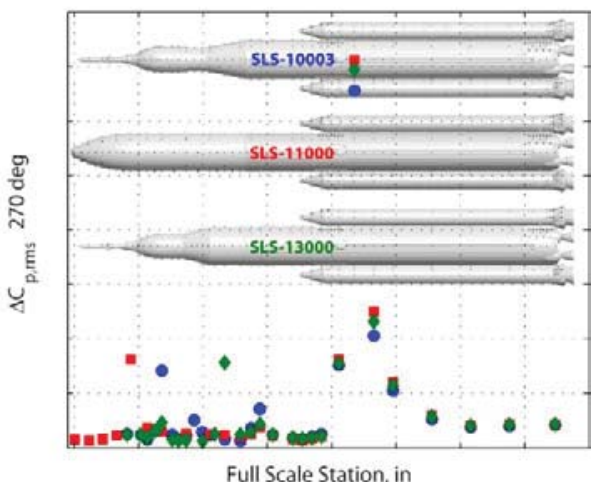

Figure 27. Comparison of $\Delta C_{p, r m s}$ on the core for SLS-10003, SLS-11000, and SLS-13000 configurations at 0/90/180/270 degrees azimuth at Mach 0.90 and $\theta=0$ deg and $\phi=0$ deg.

American Institute of Aeronautics and Astronautics 


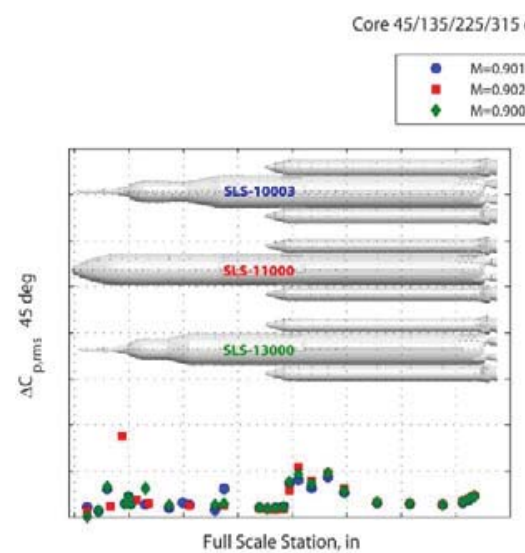

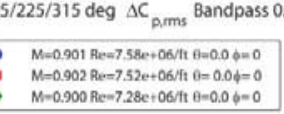
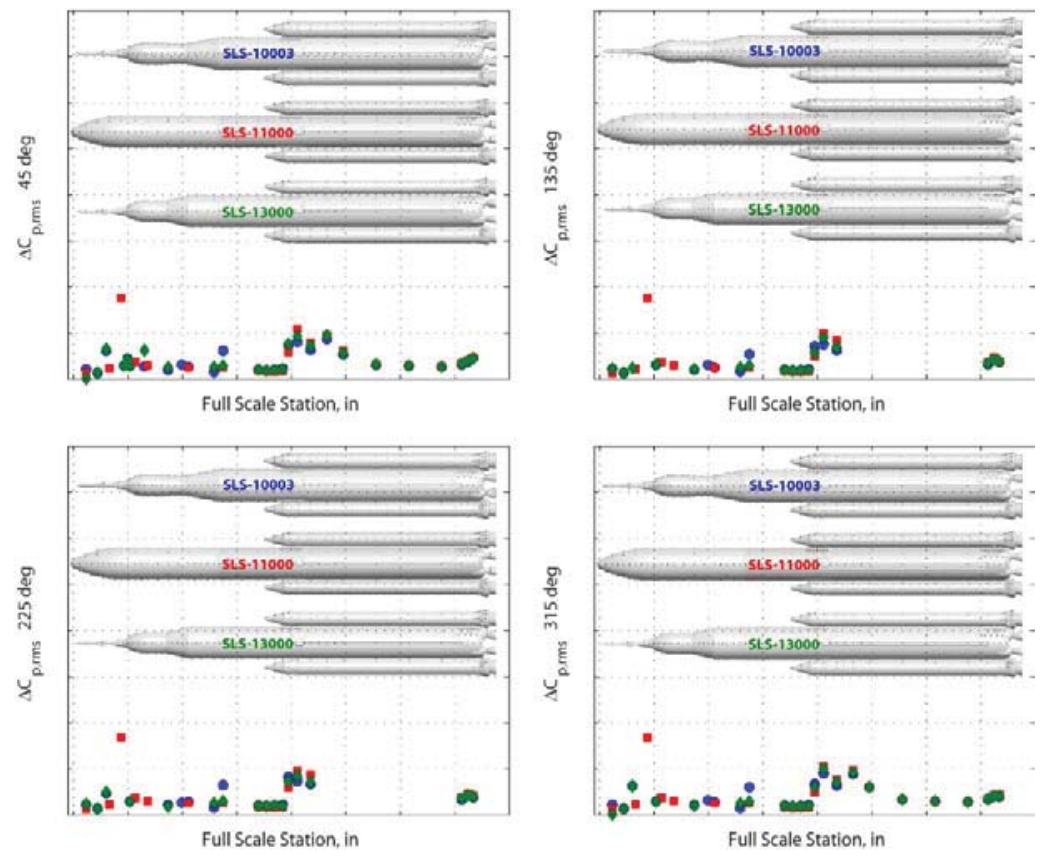

Figure 28. Comparison of $\Delta C_{p, r m s}$ on the core for SLS-10003, SLS-11000, and SLS-13000 configurations at 45/135/225/315 degrees azimuth at Mach 0.90 and $\theta=0$ deg and $\phi=0 \mathrm{deg}$.
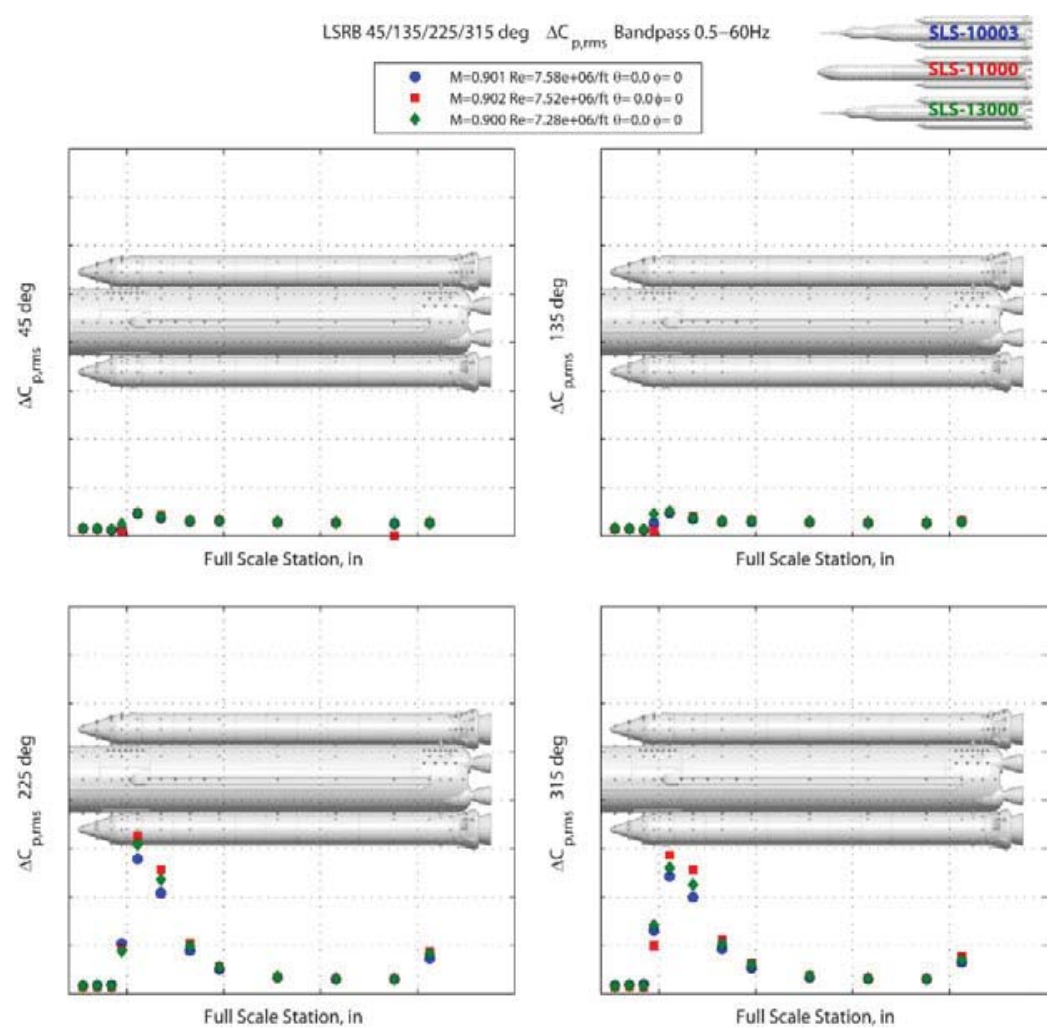

Full Scale Station, in

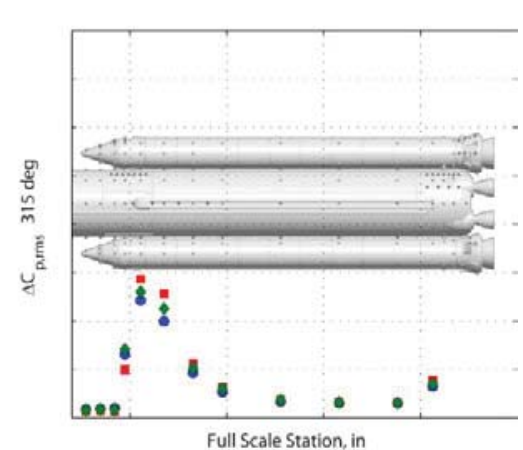

Figure 29. Comparison of $\Delta C_{p \text {,rms }}$ on the LSRB for SLS-10003, SLS-11000, and SLS-13000 configurations at 45/135/225/315 degrees azimuth at Mach 0.90 and $\theta=0$ deg and $\phi=0$ deg. 

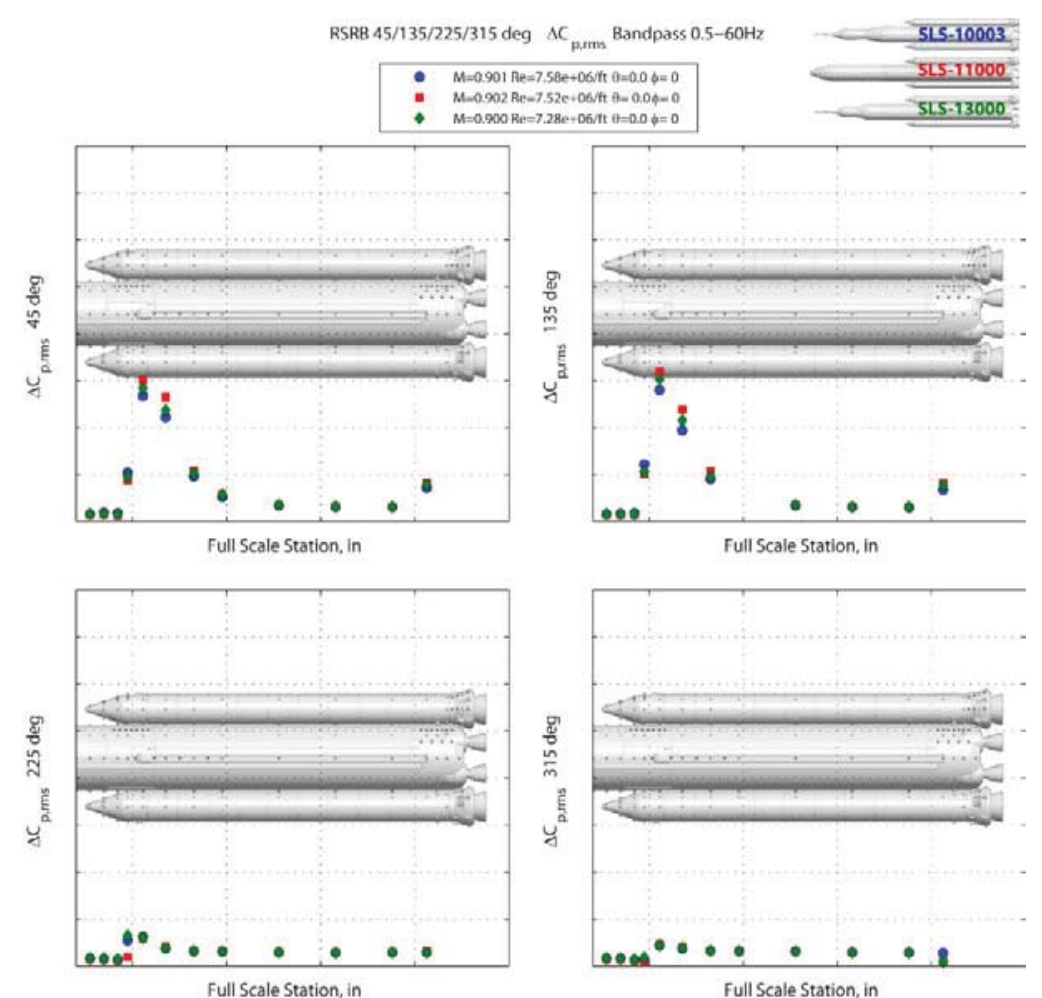

Figure 30. Comparison of $\Delta C_{p, \text { rms }}$ on the RSRB for SLS-10003, SLS-11000, and SLS-13000 configurations at 45/135/225/315 degrees azimuth at Mach 0.90 and $\theta=0$ deg and $\phi=0$ deg.

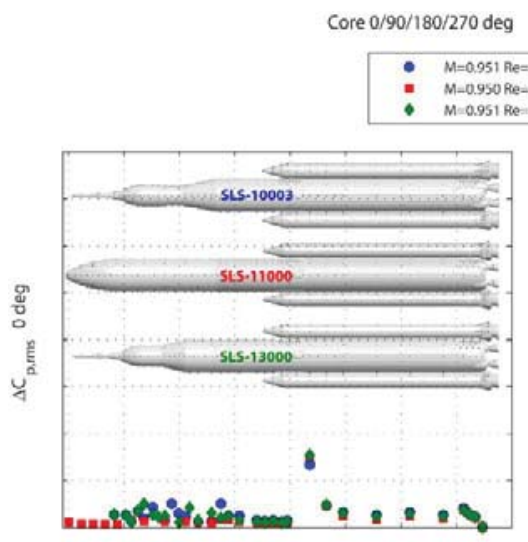

Full Scale Station, in

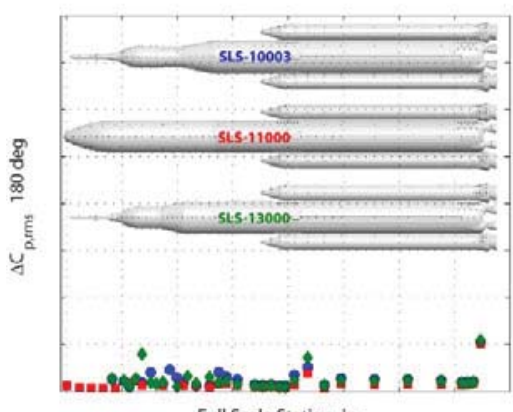

Full Scale Station, in

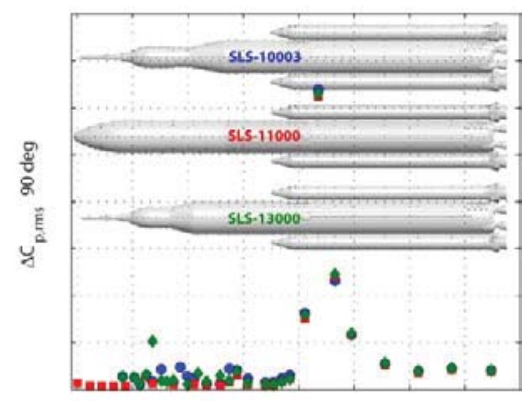

Full Scale Station, in

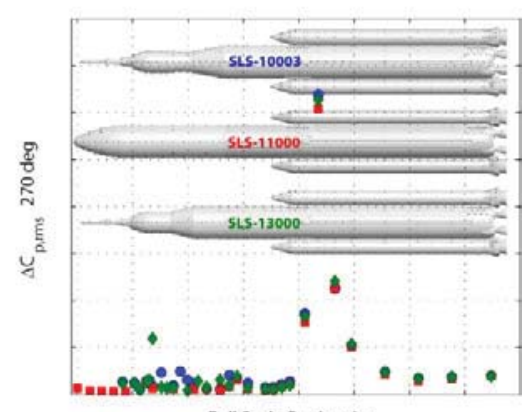

Full Scale Station, in

Figure 31. Comparison of $\Delta C_{p, r m s}$ on the core for SLS-10003, SLS-11000, and SLS-13000 configurations at 0/90/180/270 degrees azimuth at Mach 0.95 and $\theta=0$ deg and $\phi=0 \mathrm{deg}$. 


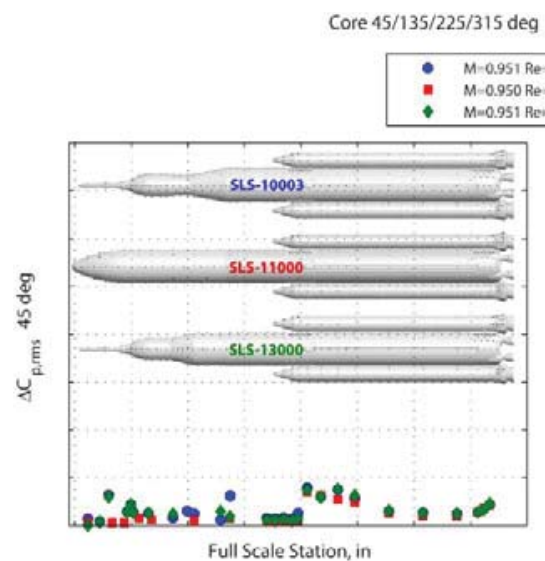

p.rms Bandpass $0.5-60 \mathrm{~Hz}$

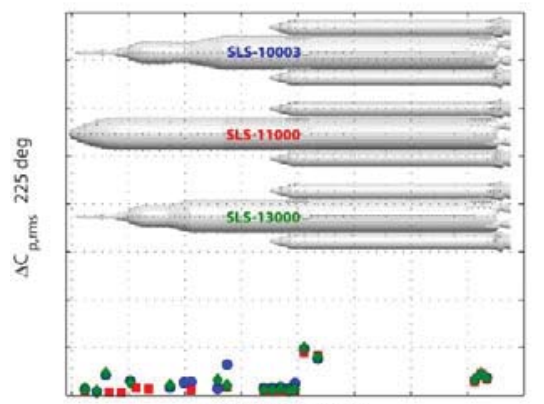

Full Scale Station, in

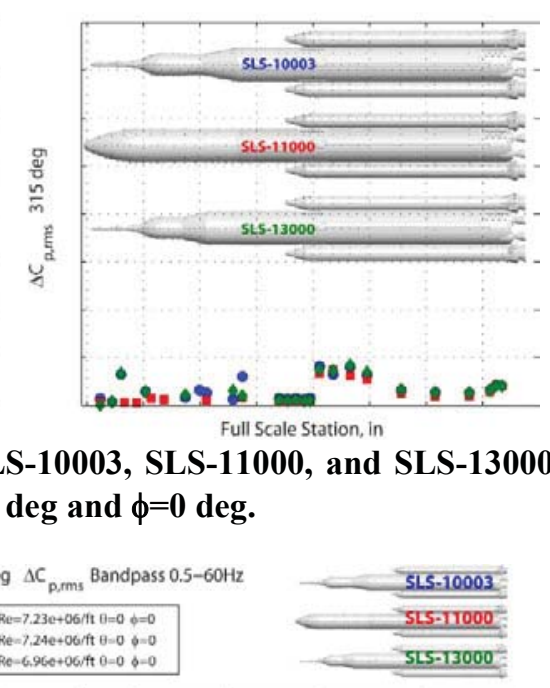

Figure 32. Comparison of $\Delta C_{p, r m s}$ on the core for SLS-10003, SLS-11000, and SLS-13000 configurations at 45/135/225/315 degrees azimuth at Mach 0.95 and $\theta=0$ deg and $\phi=0$ deg.
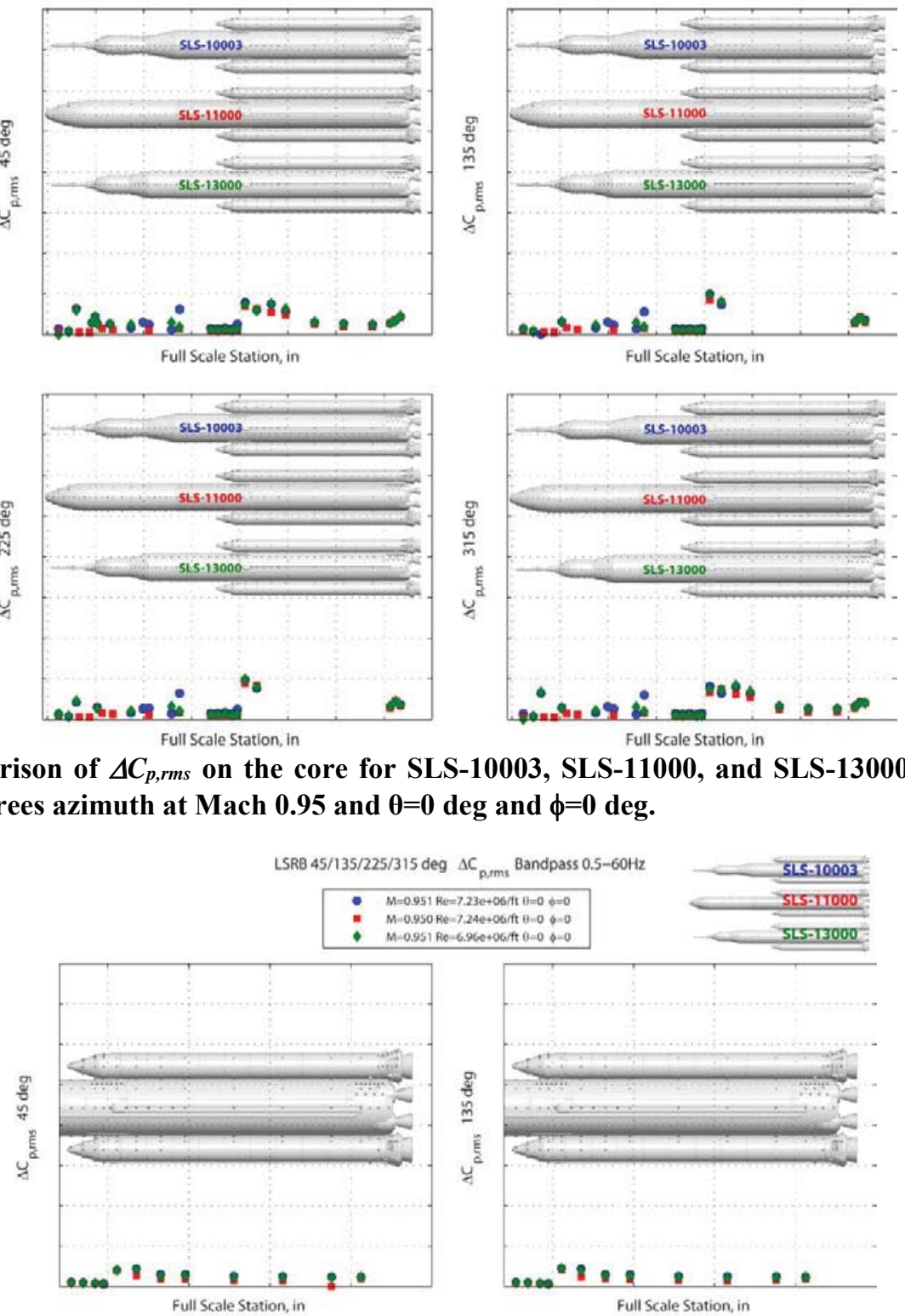

Full Scale Station, in
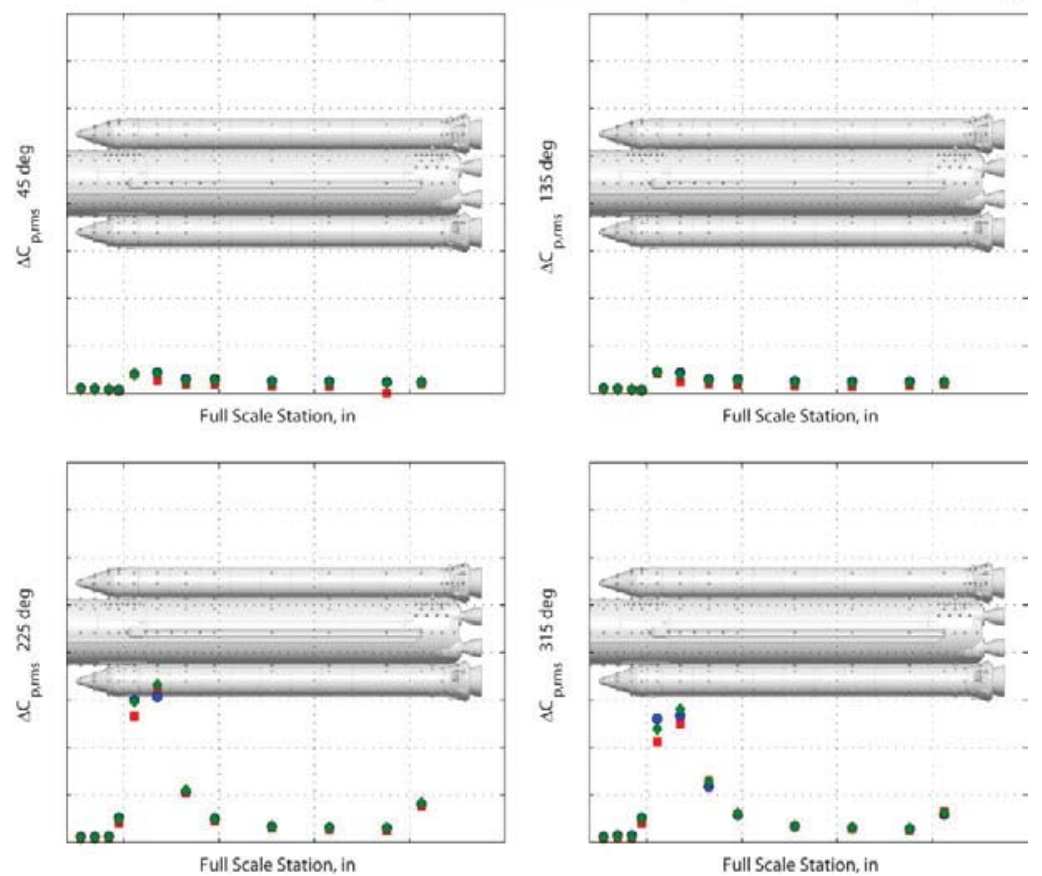

Figure 33. Comparison of $\Delta C_{p, r m s}$ on the LSRB for SLS-10003, SLS-11000, and SLS-13000 configurations at 45/135/225/315 degrees azimuth at Mach 0.95 and $\theta=0$ deg and $\phi=0$ deg. 

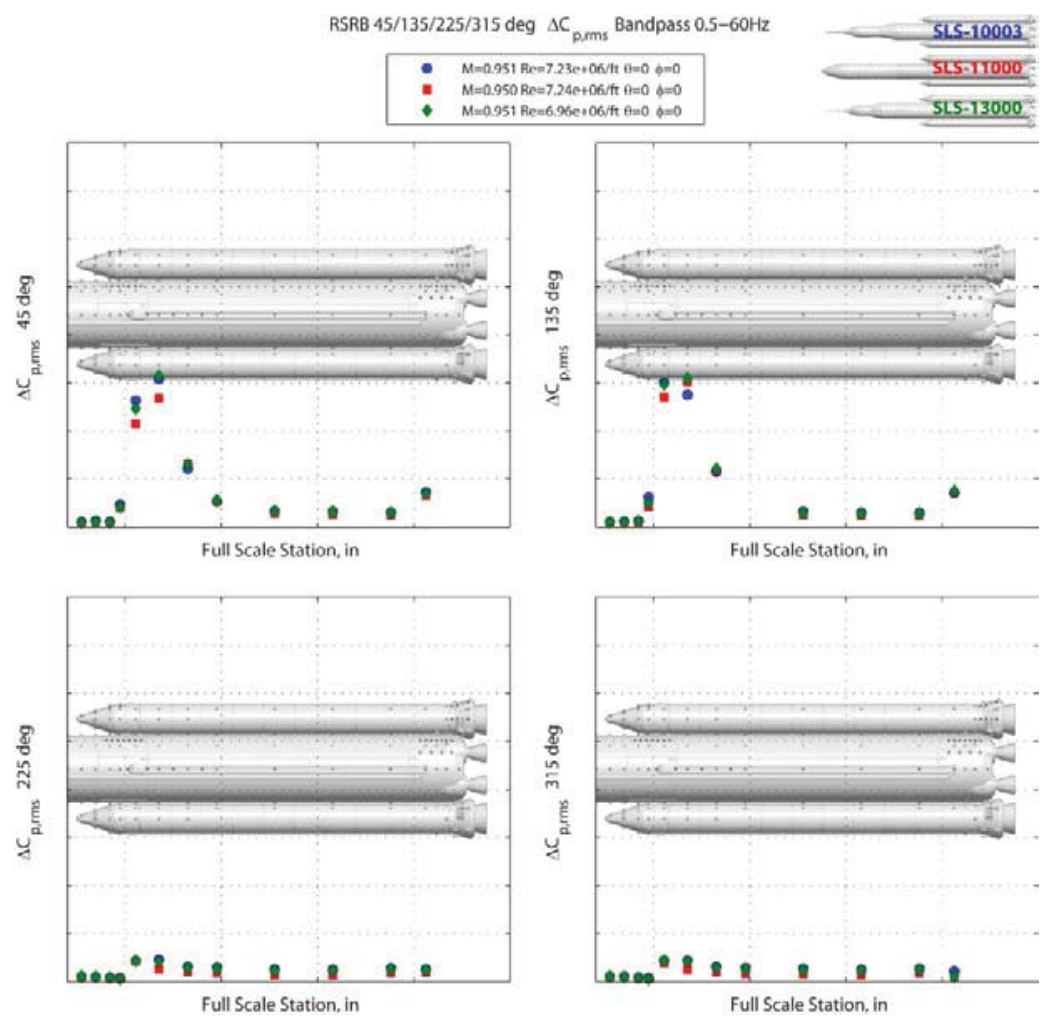

Figure 34. Comparison of $\Delta C_{p \text {,rms }}$ on the RSRB for SLS-10003, SLS-11000, and SLS-13000 configurations at 45/135/225/315 degrees azimuth at Mach 0.95 and $\theta=0$ deg and $\phi=0$ deg.

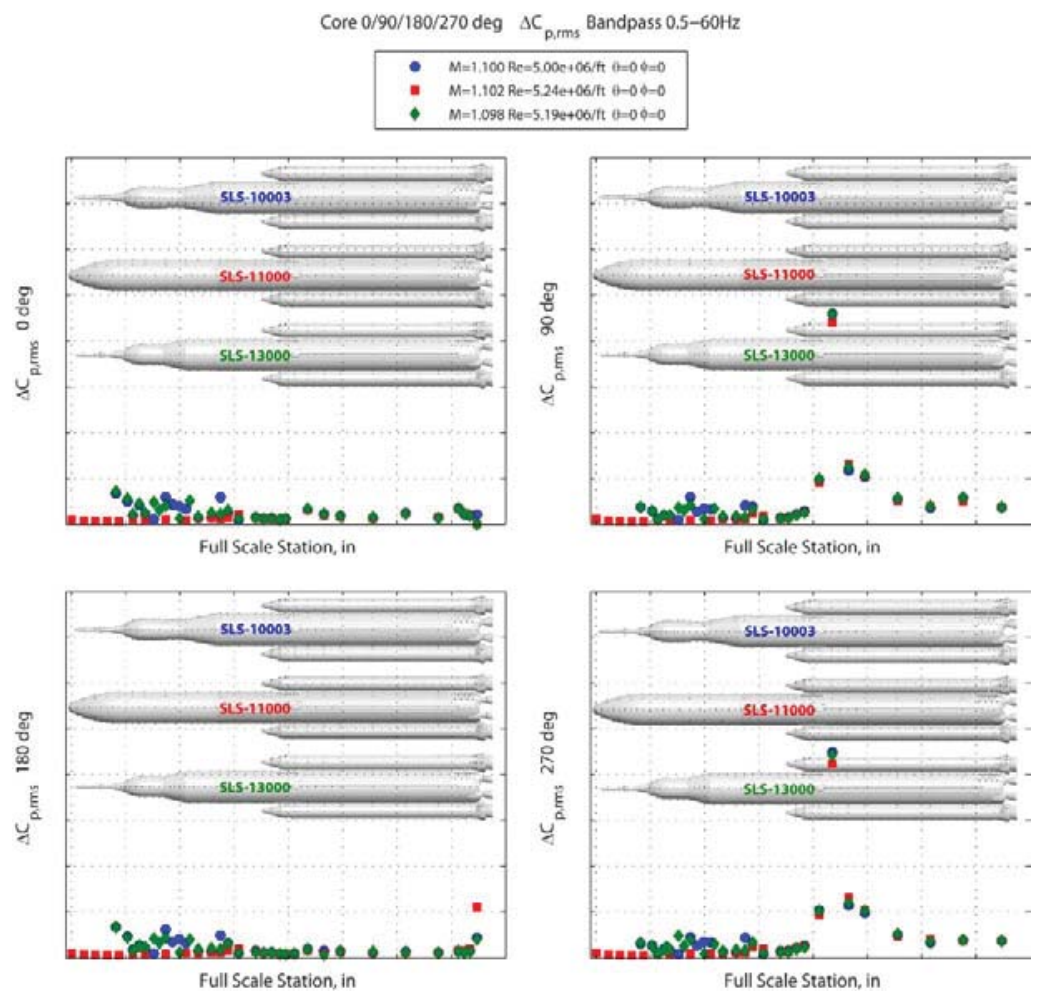

Figure 35. Comparison of $\Delta C_{p, r m s}$ on the core for SLS-10003, SLS-11000, and SLS-13000 configurations at 0/90/180/270 degrees azimuth at Mach 1.10 and $\theta=0$ deg and $\phi=0$ deg. 


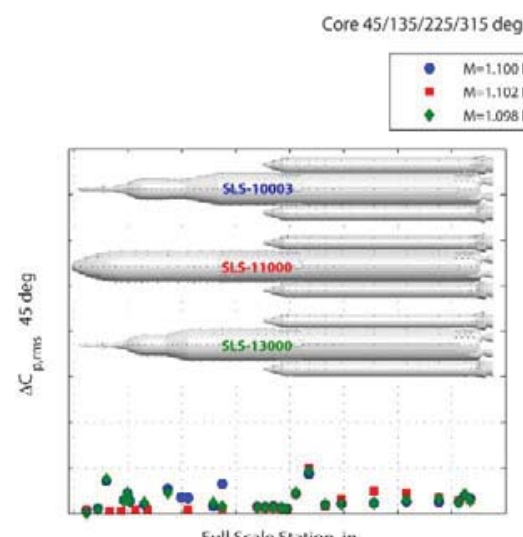

B.ms Bandpass $0.5-60 \mathrm{~Hz}$

Full Scale Station, in
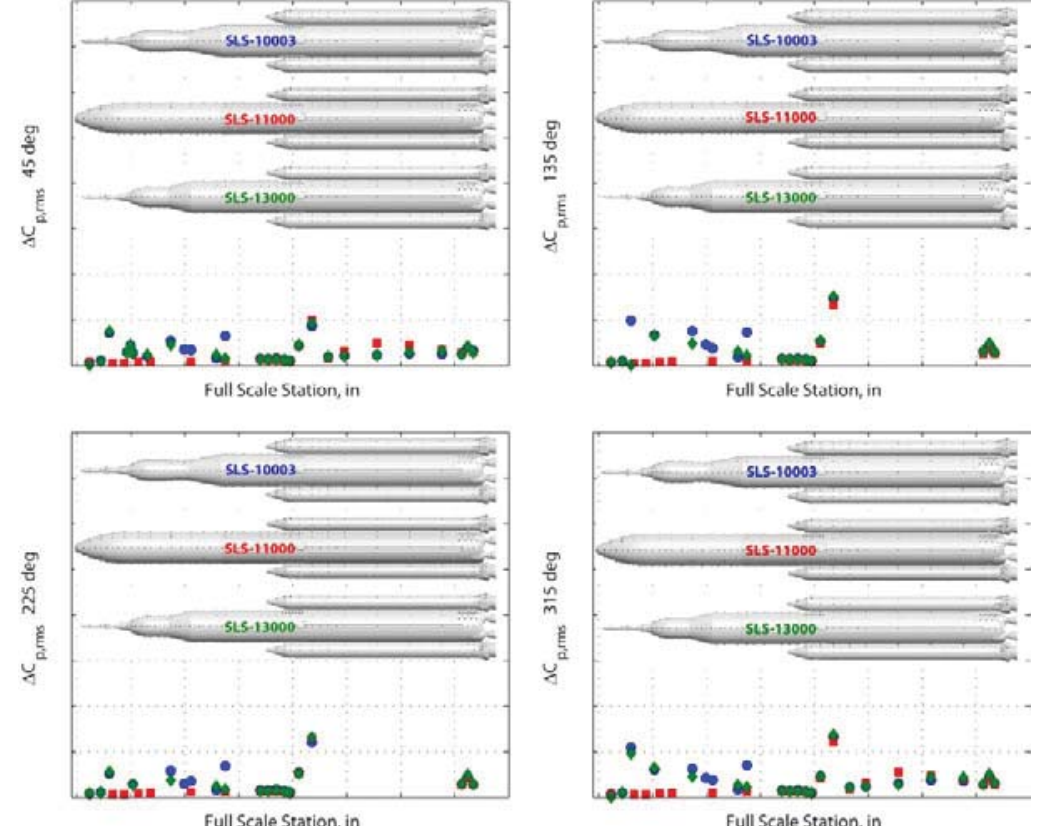

Full Scale Station, in

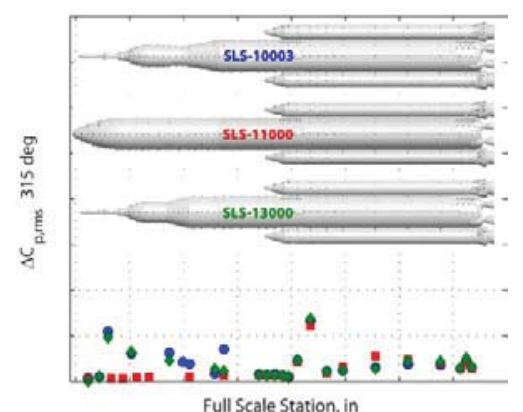

Figure 36. Comparison of $\Delta C_{p, r m s}$ on the core for SLS-10003, SLS-11000, and SLS-13000 configurations at 45/135/225/315 degrees azimuth at Mach 1.10 and $\theta=0$ deg and $\phi=0$ deg.
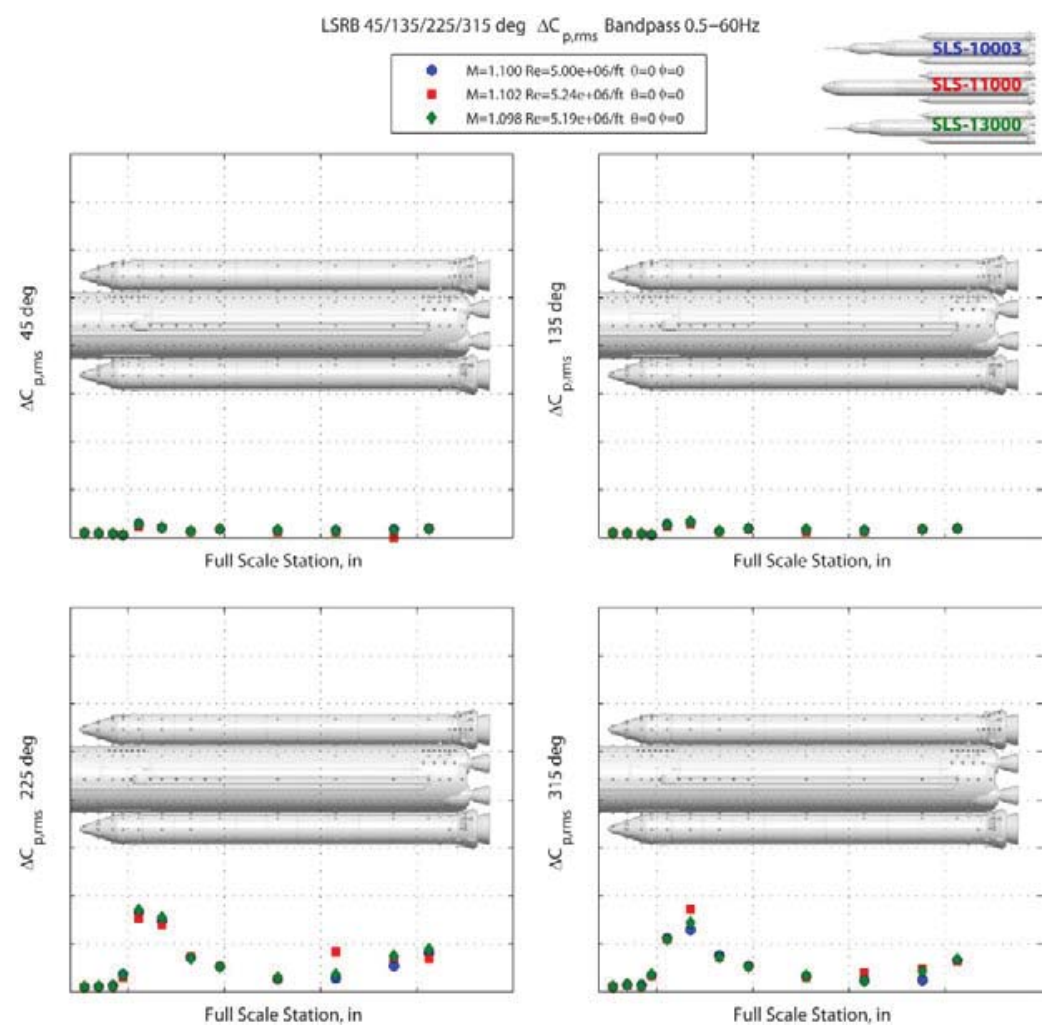

Figure 37. Comparison of $\Delta C_{p \text {,rms }}$ on the LSRB for SLS-10003, SLS-11000, and SLS-13000 configurations at 45/135/225/315 degrees azimuth at Mach 1.10 and $\theta=0$ deg and $\phi=0$ deg. 

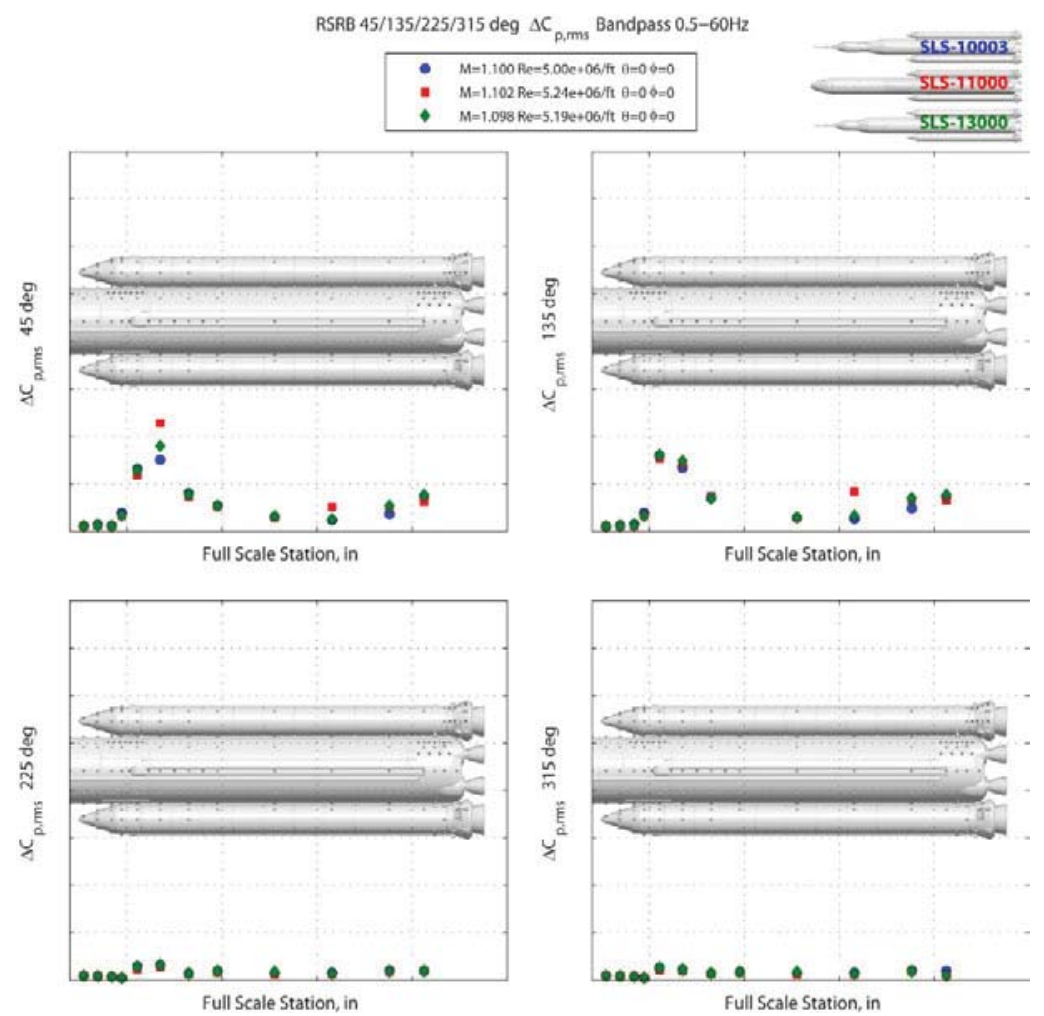

Figure 38. Comparison of $\Delta C_{p \text {,rms }}$ on the RSRB for SLS-10003, SLS-11000, and SLS-13000 configurations at 45/135/225/315 degrees azimuth at Mach 1.10 and $\theta=0$ deg and $\phi=0$ deg.
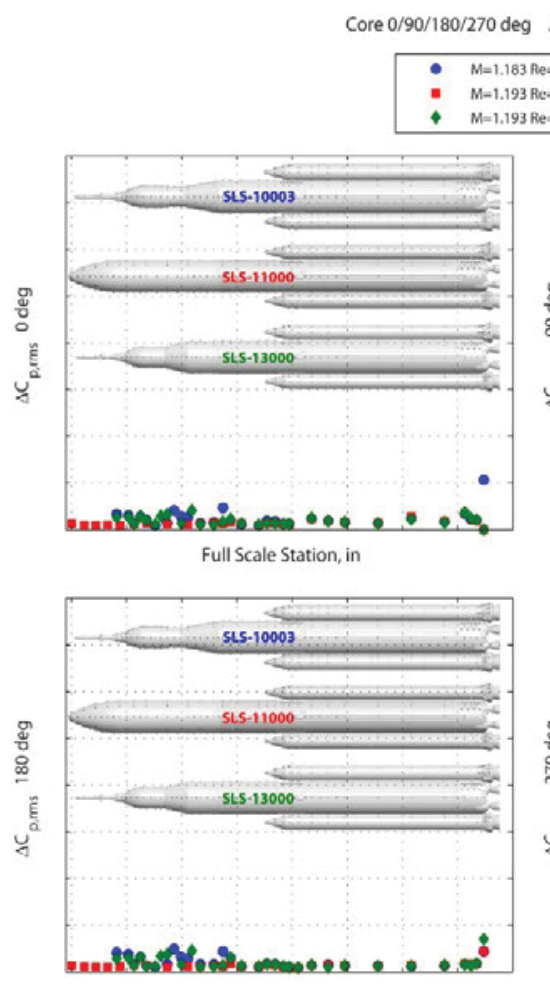

Full Scale Station, in

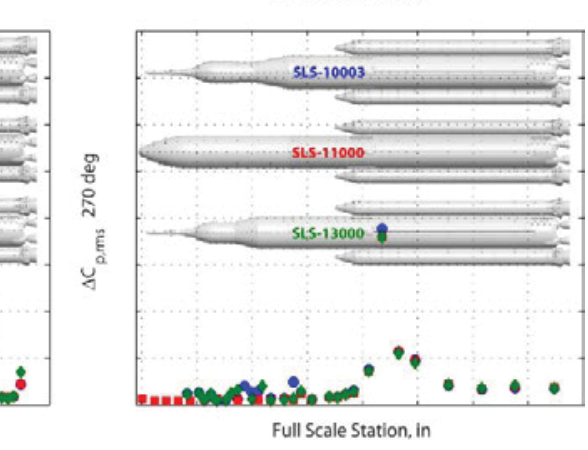

Figure 39. Comparison of $\Delta C_{p, r m s}$ on the core for SLS-10003, SLS-11000, and SLS-13000 configurations at 0/90/180/270 degrees azimuth at Mach 1.19 and $\theta=0$ deg and $\phi=0$ deg. 


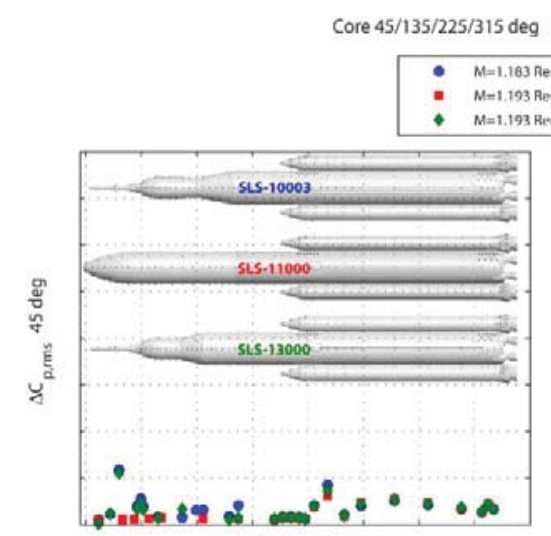

p.ms Bandpass $0.5-60 \mathrm{~Hz}$
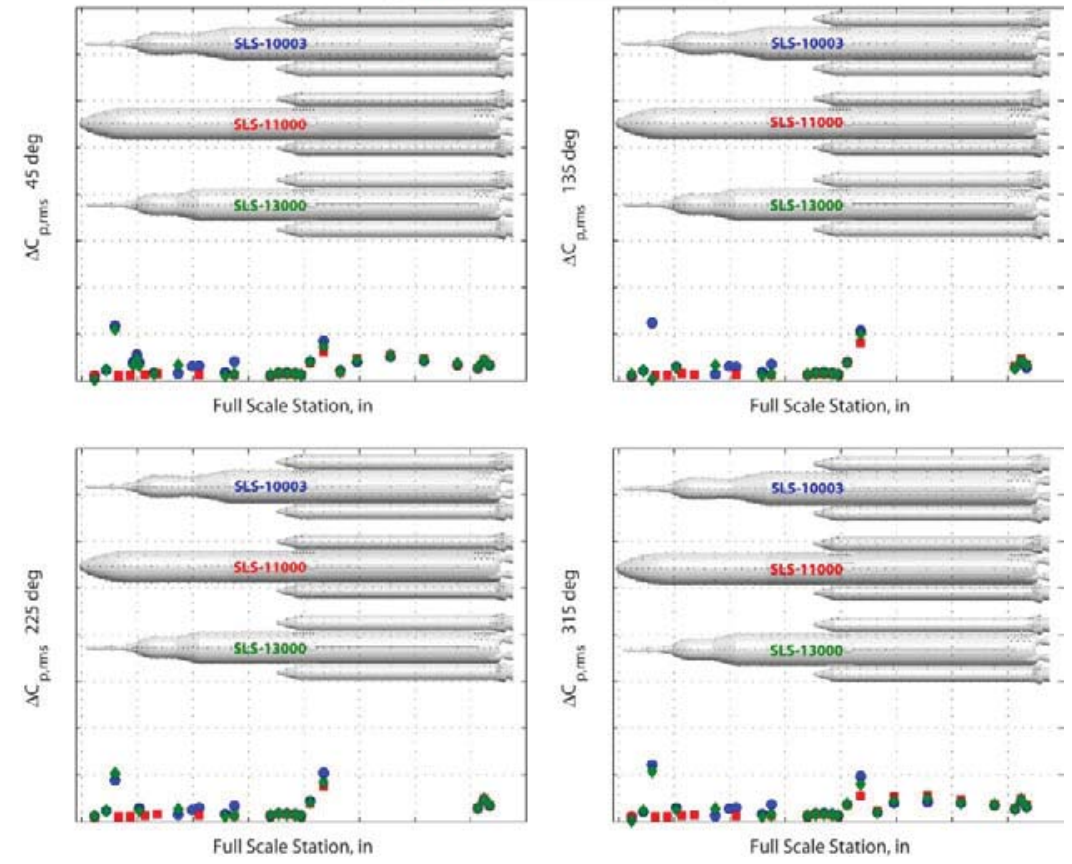

Full Scale Station, in

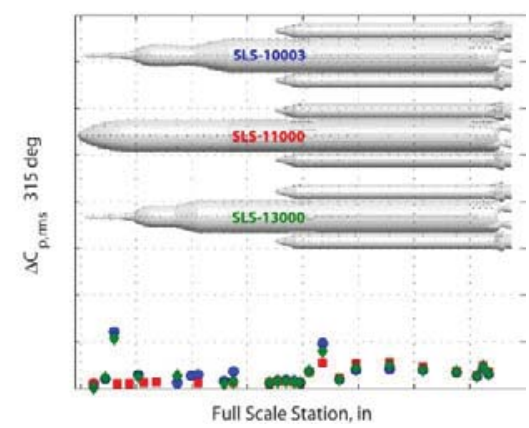

Figure 40. Comparison of $\Delta C_{p, r m s}$ on the core for SLS-10003, SLS-11000, and SLS-13000 configurations at 45/135/225/315 degrees azimuth at Mach 1.19 and $\theta=0$ deg and $\phi=0$ deg.
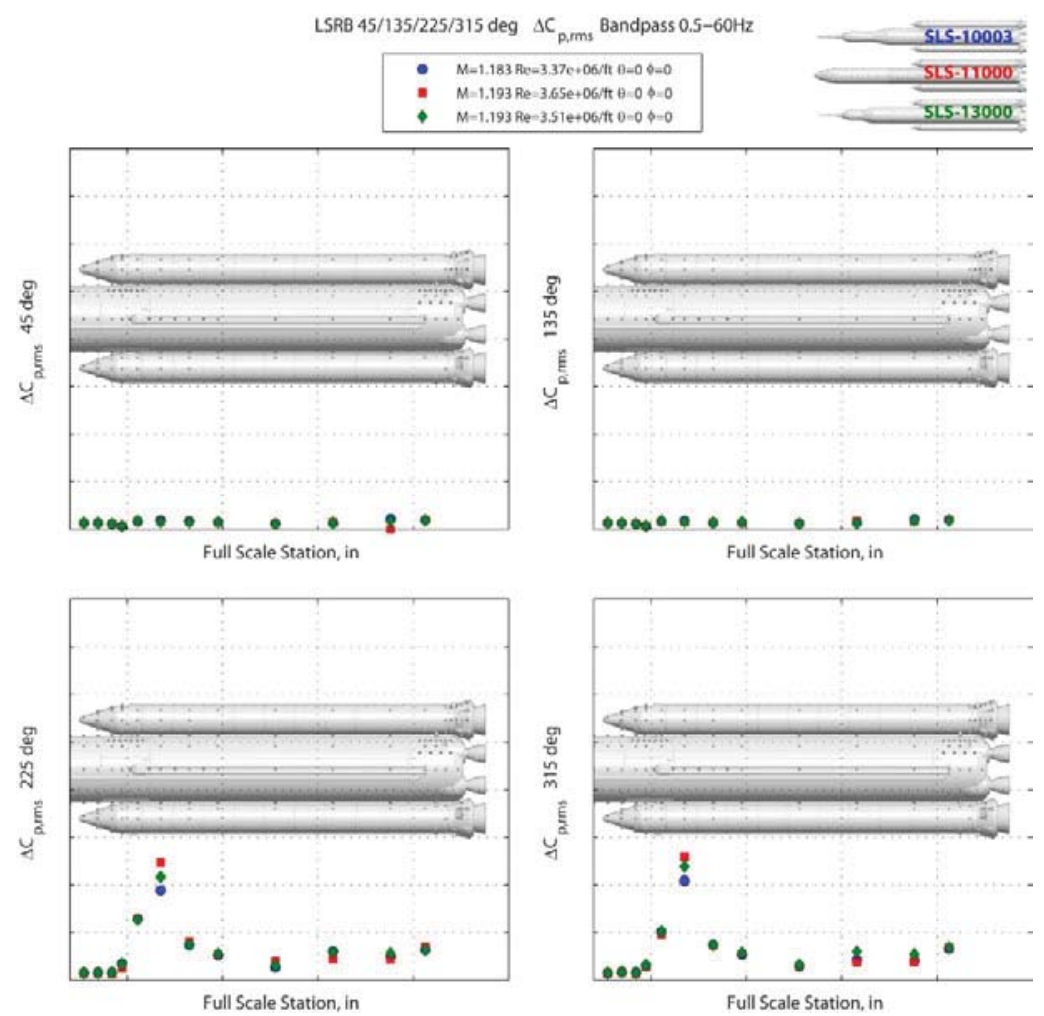

Figure 41. Comparison of $\Delta C_{p \text {,rms }}$ on the LSRB for SLS-10003, SLS-11000, and SLS-13000 configurations at 45/135/225/315 degrees azimuth at Mach 1.19 and $\theta=0$ deg and $\phi=0$ deg. 

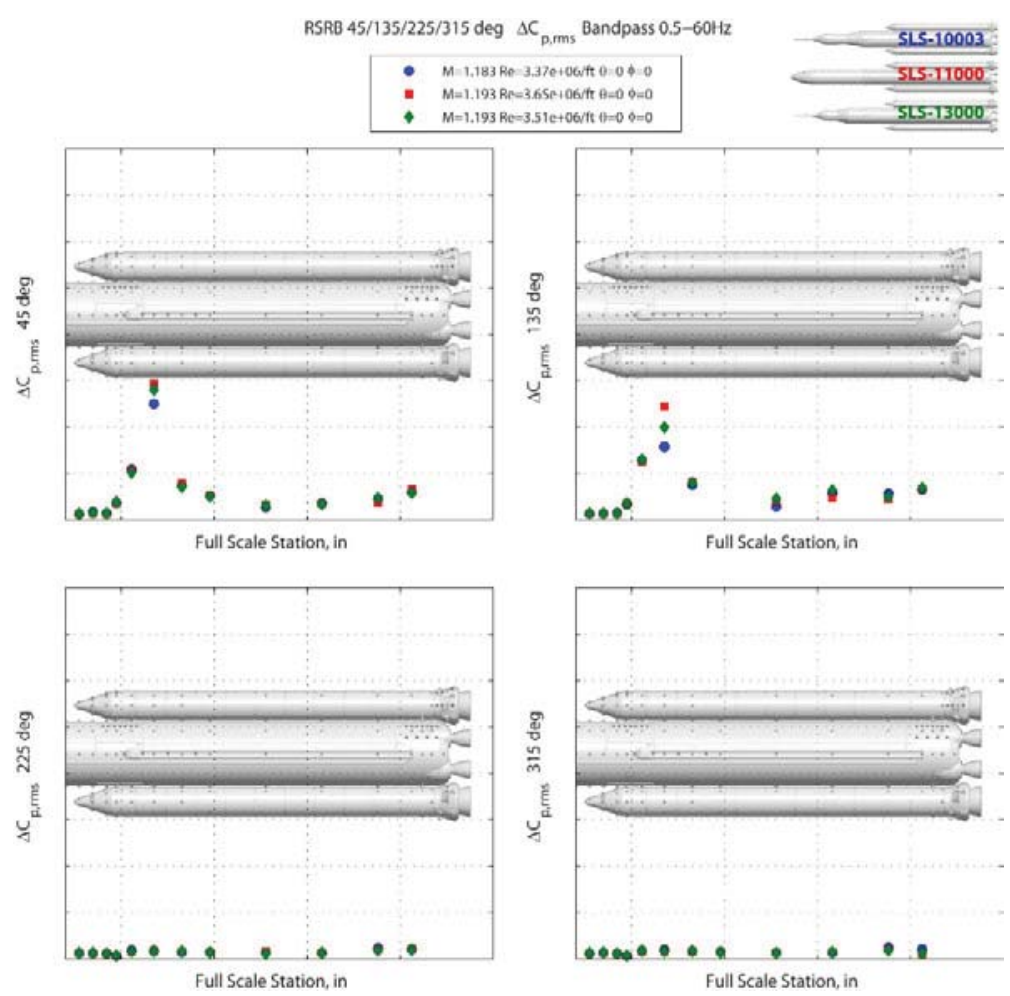

Figure 42. Comparison of $\Delta C_{p, r m s}$ on the RSRB for SLS-10003, SLS-11000, and SLS-13000 configurations at 45/135/225/315 degrees azimuth at Mach 1.19 and $\theta=0 \mathrm{deg}$ and $\phi=0 \mathrm{deg}$.

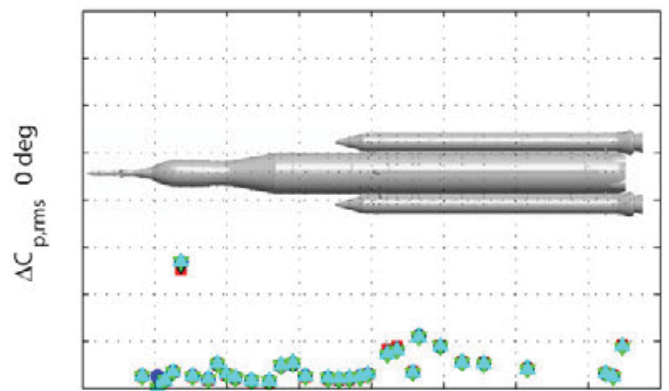

Vehicle Station

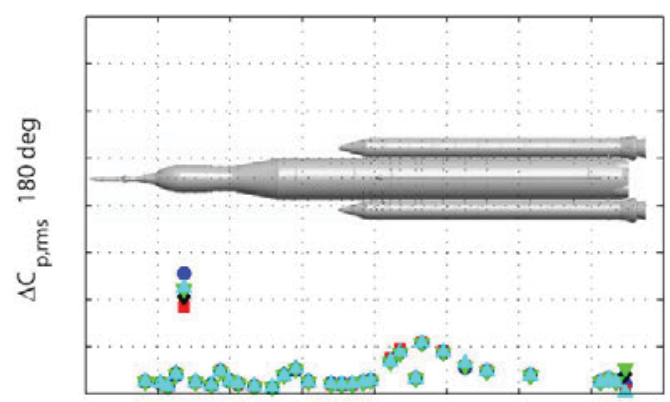

Vehicle Station

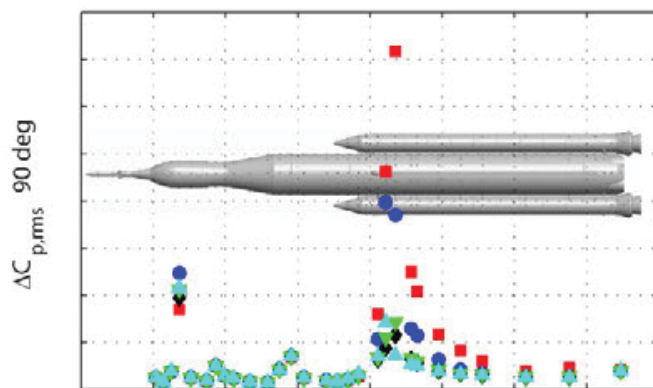

Vehicle Station

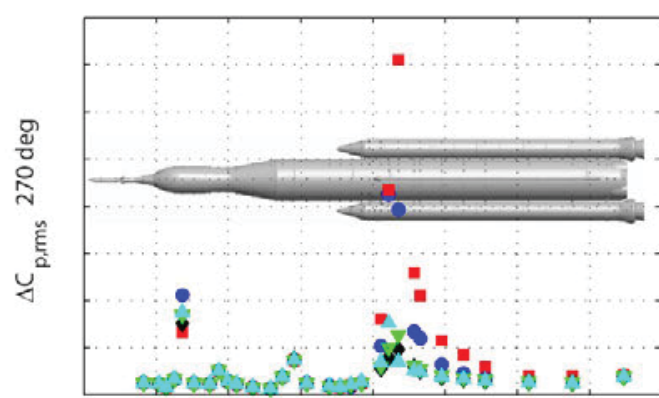

Vehicle Station

SLS-10005 Core $0 / 90 / 180 / 270 \mathrm{deg}$ Bandpass $0.5-60 \mathrm{~Hz}$

$M=0.90 \quad \theta=0$ deg $\phi=0$ deg

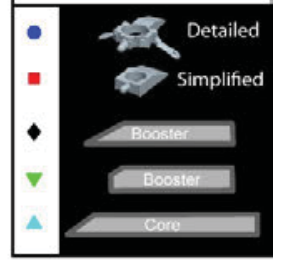

Figure 43. $\Delta C_{p, r m s}$ on the core at $0 / 90 / 180 / 270$ degrees azimuth at Mach 0.90 for simplified and detailed booster forward attachment with comparison to fence BMOs.

American Institute of Aeronautics and Astronautics 


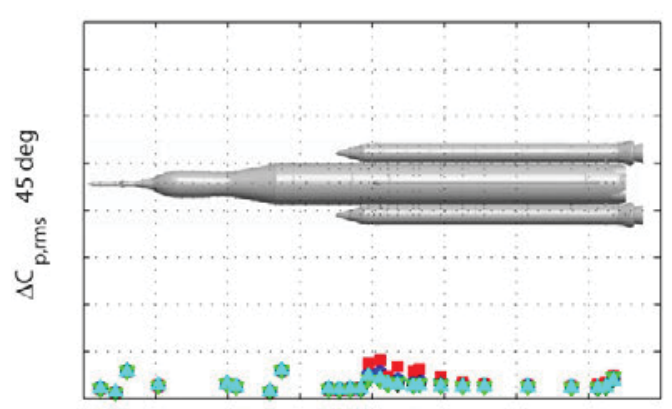

Vehicle Station

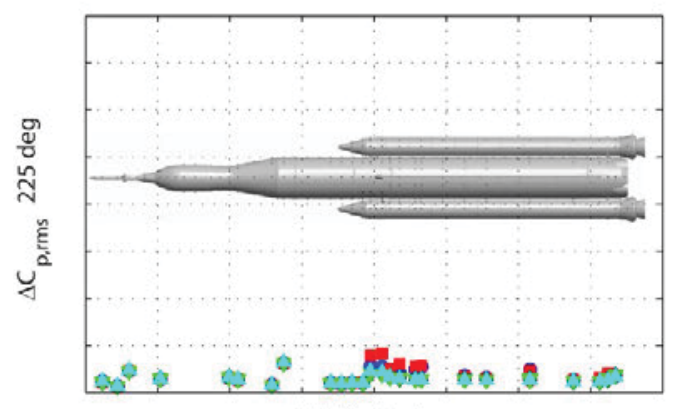

Vehicle Station

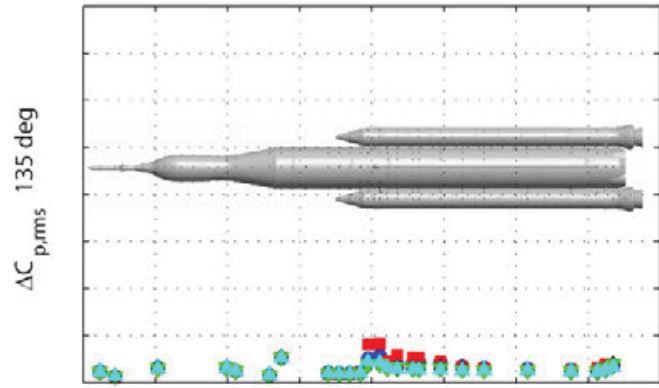

Vehicle Station

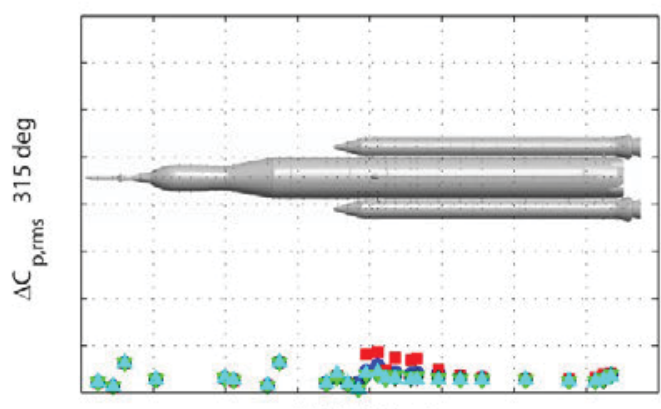

Vehicle Station
SLS-10005 Core $45 / 135 / 225 / 315 \mathrm{deg}$ Bandpass $0.5-60 \mathrm{~Hz}$

$M=0.90 \quad \theta=0$ deg $\phi=0$ deg

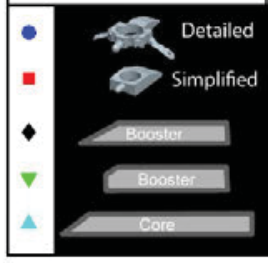

Figure 44. $\Delta C_{p, r m s}$ on the core at 45/135/225/315 degrees azimuth at Mach 0.90 for simplified and detailed booster forward attachment with comparison to fence BMOs.

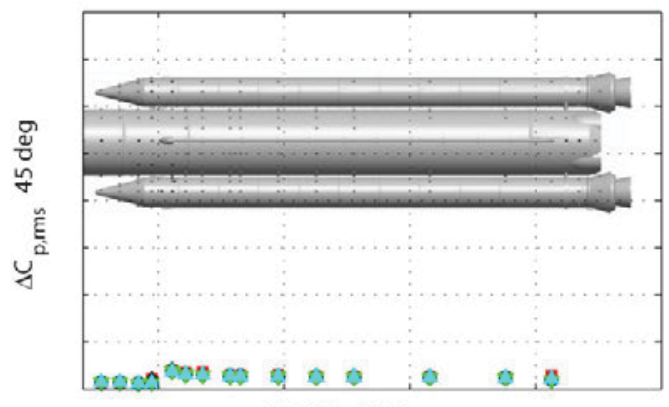

Vehicle Station

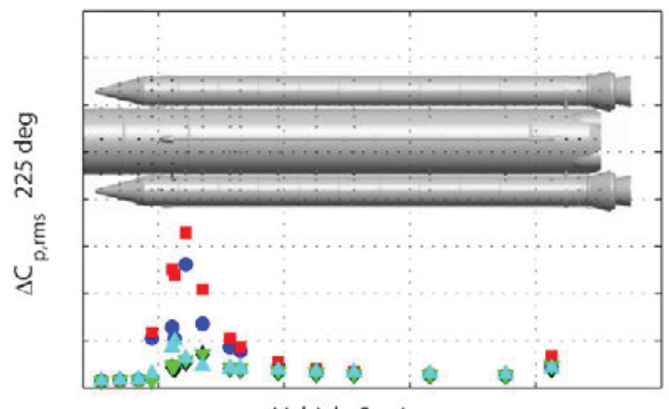

Vehicle Station

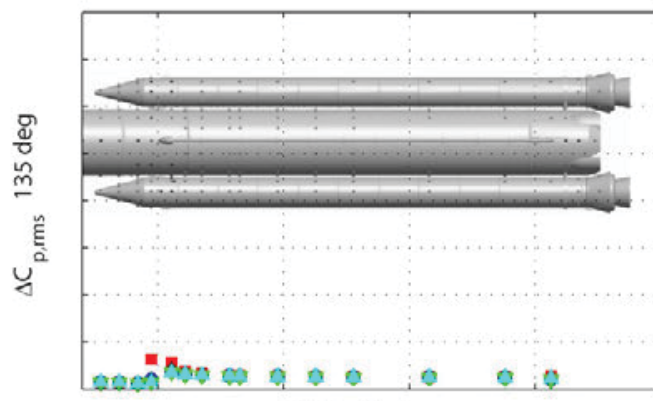

Vehicle Station

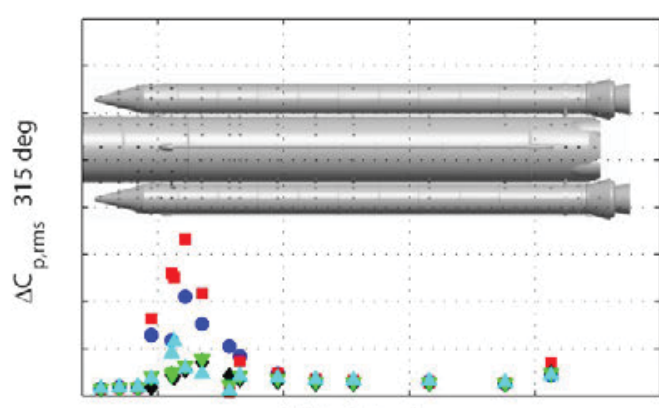

Vehicle Station
SLS-10005 LSRB 45/135/225/315 deg

Bandpass $0.5-60 \mathrm{~Hz}$

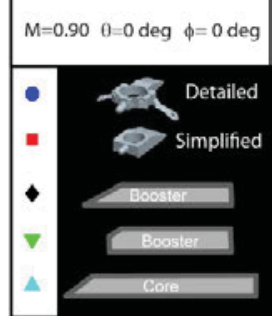

Figure 45. $\Delta C_{p, r m s}$ on the LSRB at 45/135/225/315 degrees azimuth at Mach 0.90 for simplified and detailed booster forward attachment with comparison to fence BMOs. 

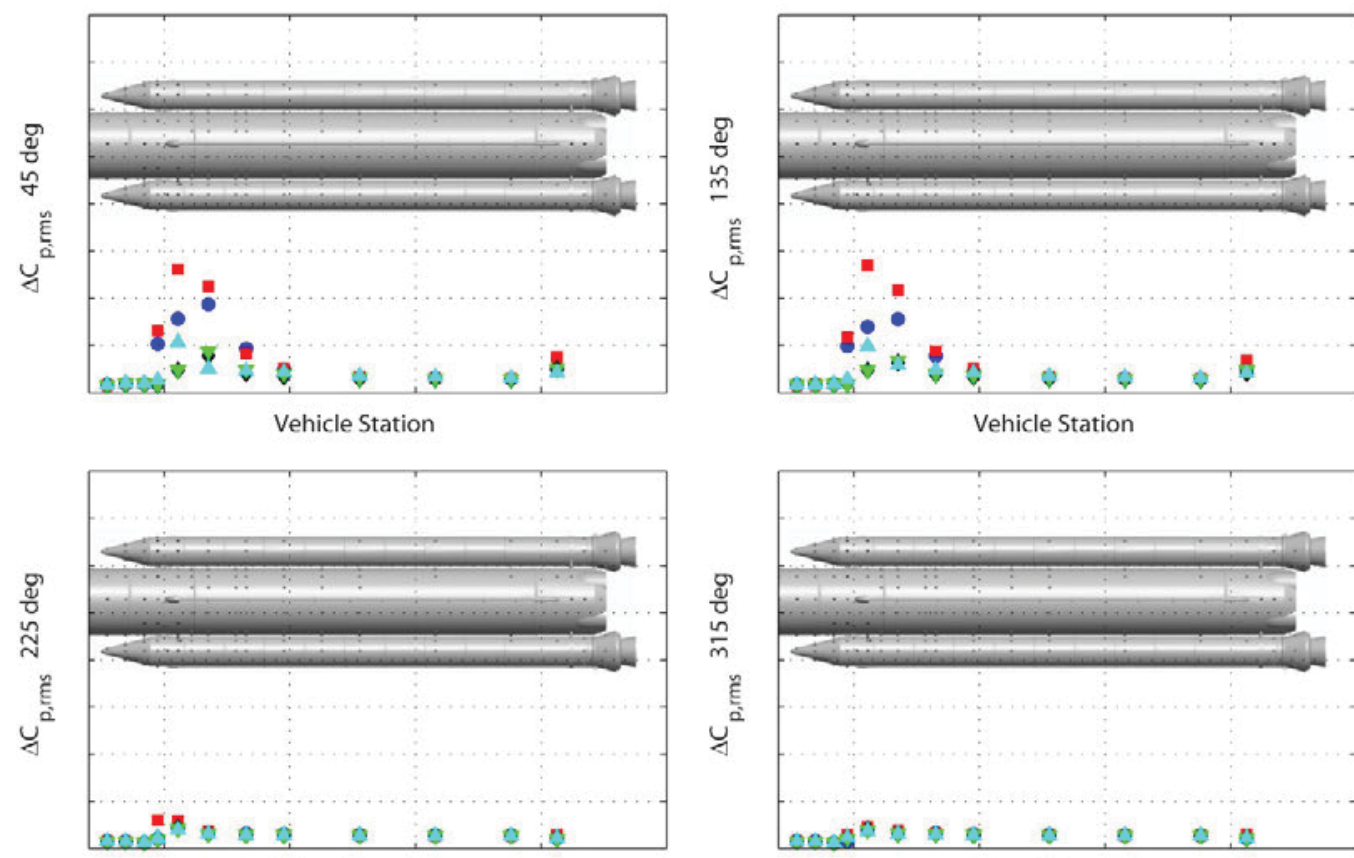

SLS-10005 RSRB $45 / 135 / 225 / 315$ deg

Bandpass $0.5-60 \mathrm{~Hz}$

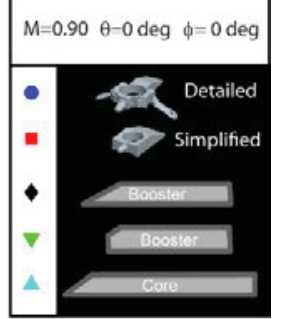

Vehicle Station

Vehicle Station

Figure 46. $\Delta C_{p, r m s}$ on the RSRB at 45/135/225/315 degrees azimuth at Mach 0.90 for simplified and detailed booster forward attachment with comparison to fence BMOs.

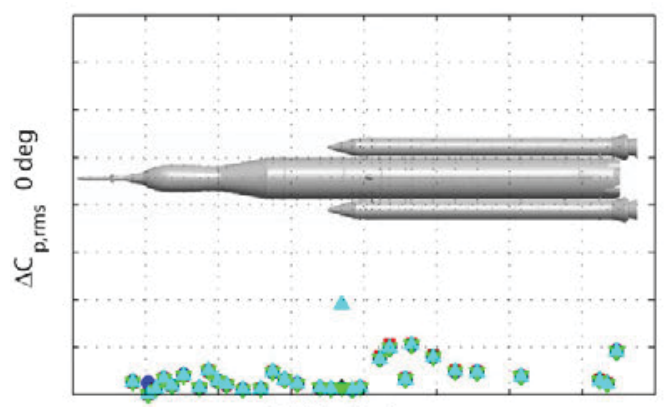

Vehicle Station

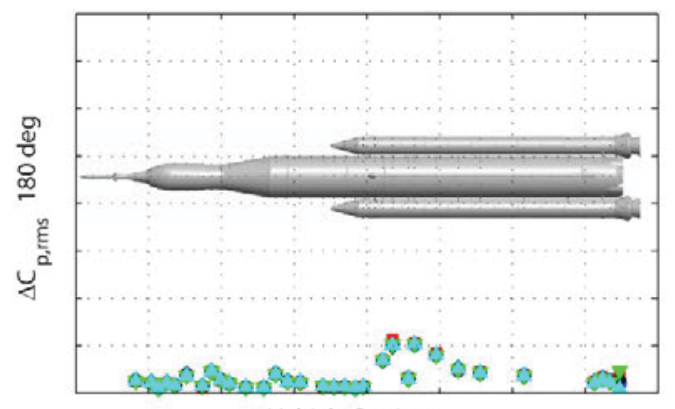

Vehicle Station

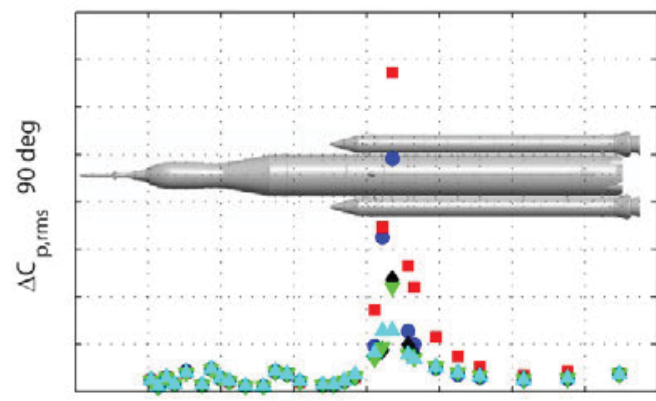

Vehicle Station

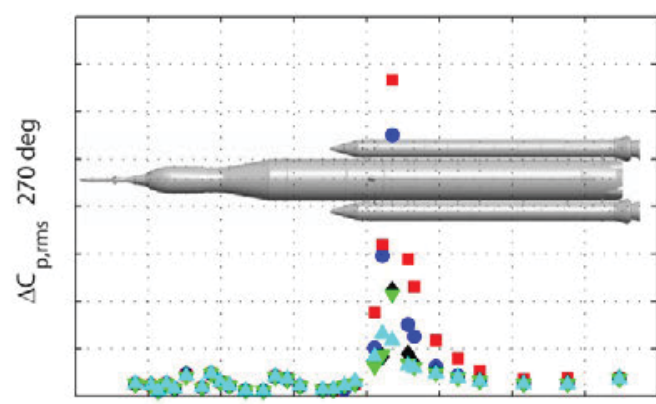

Vehicle Station
SLS-10005 Core $0 / 90 / 180 / 270 \mathrm{deg}$

Bandpass $0.5-60 \mathrm{~Hz}$

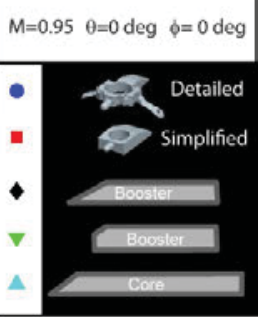

Figure 47. $\Delta C_{p, r m s}$ on the core at $0 / 90 / 180 / 270$ degrees azimuth at Mach 0.95 for simplified and detailed booster forward attachment with comparison to fence BMOs. 

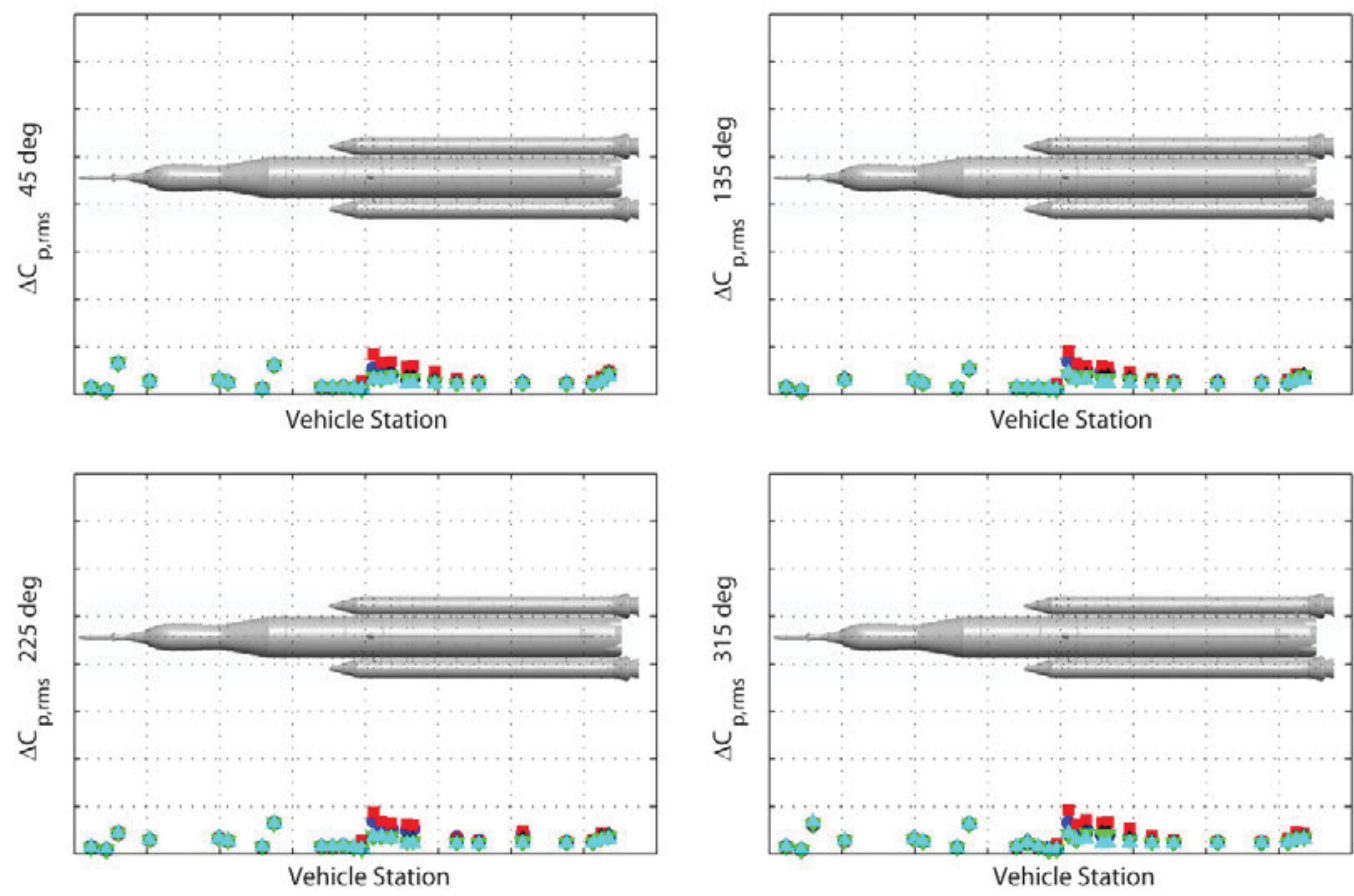

SLS-10005 Core

$45 / 135 / 225 / 315 \mathrm{deg}$

Bandpass $0.5-60 \mathrm{~Hz}$

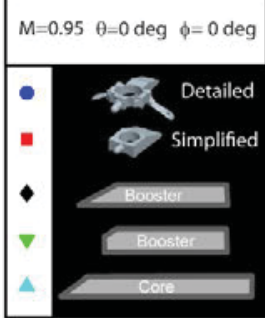

Figure 48. $\Delta C_{p, r m s}$ on the core at 45/135/225/315 degrees azimuth at Mach 0.95 for simplified and detailed booster forward attachment with comparison to fence BMOs.
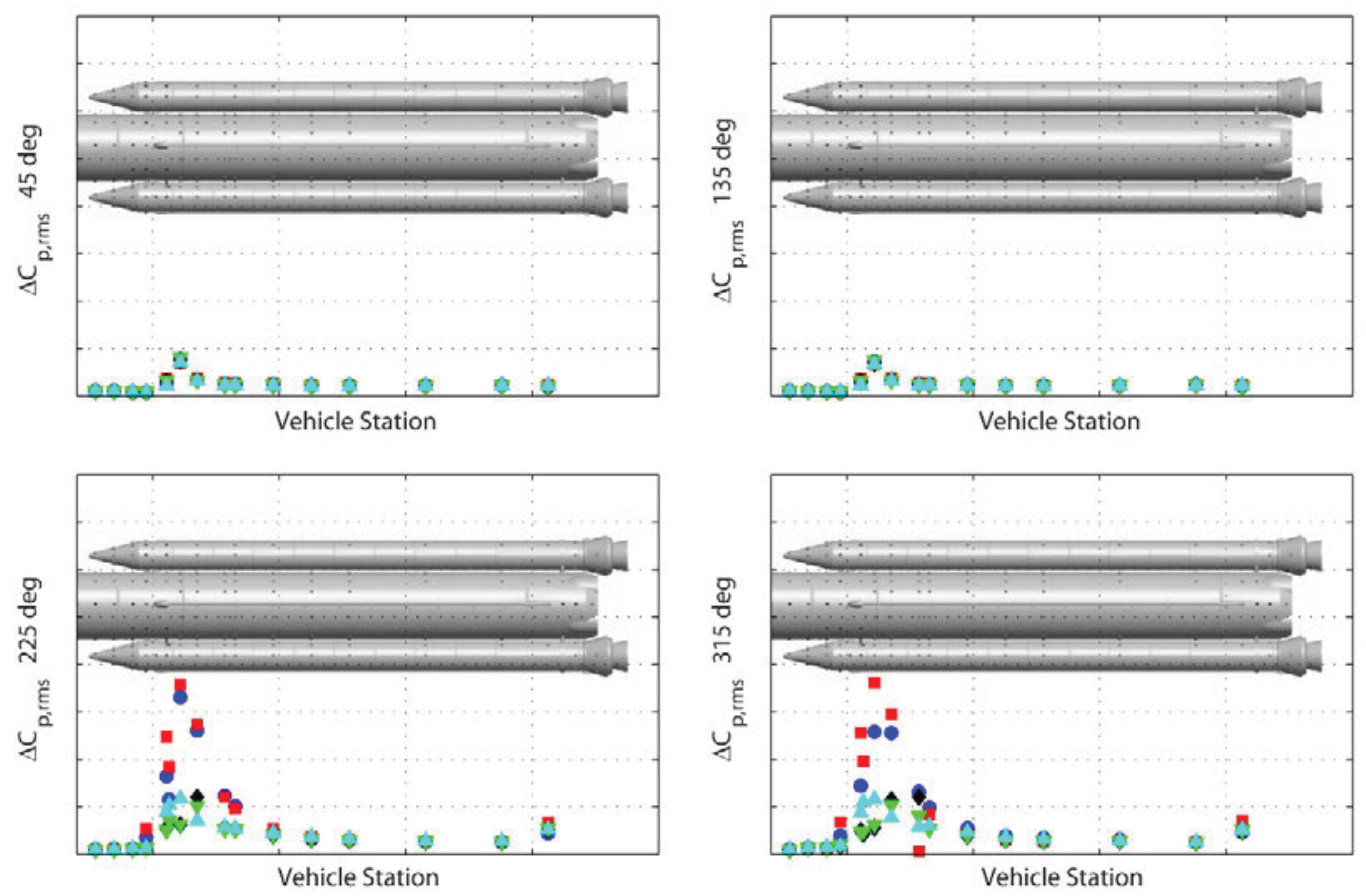

SLS-10005 LSRB

$45 / 135 / 225 / 315 \mathrm{deg}$

Bandpass $0.5-60 \mathrm{~Hz}$

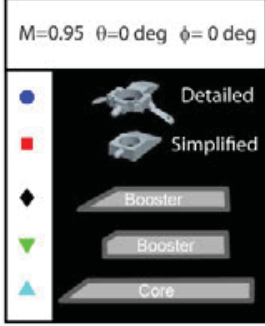

Figure 49. $\Delta C_{p, r m s}$ on the LSRB at 45/135/225/315 degrees azimuth at Mach 0.95 for simplified and detailed booster forward attachment with comparison to fence BMOs. 

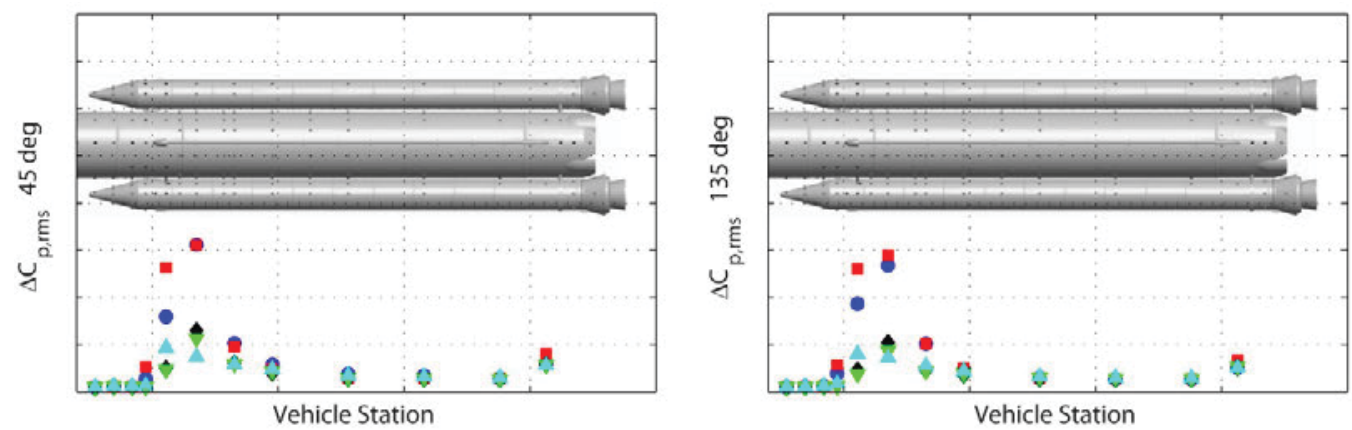

SLS-10005 RSRB

$45 / 135 / 225 / 315 \mathrm{deg}$

Bandpass $0.5-60 \mathrm{~Hz}$
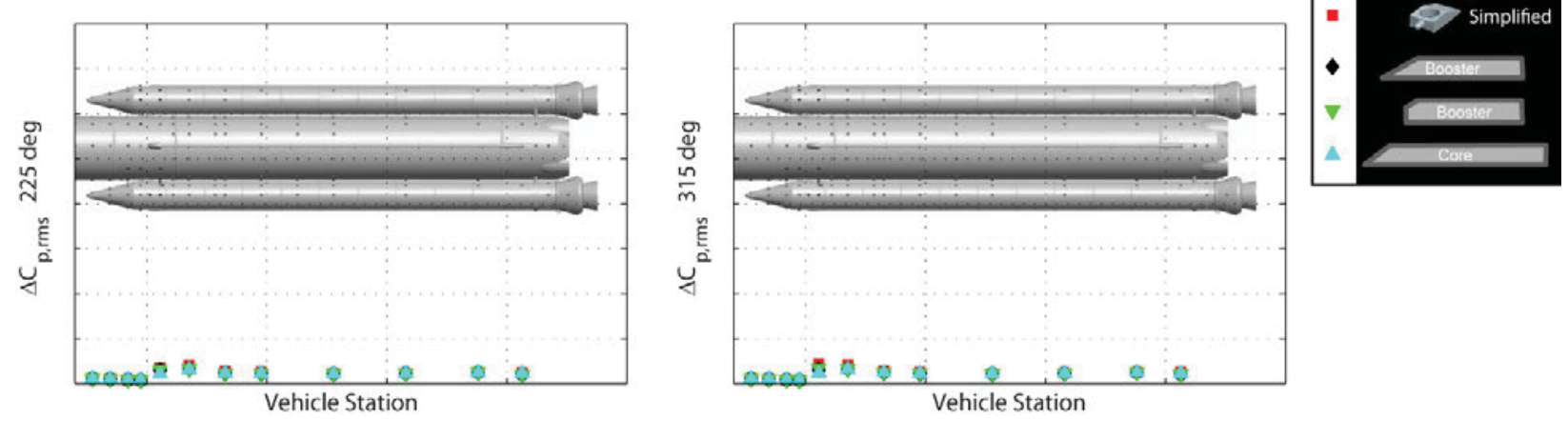

Figure 50. $\Delta C_{p, r m s}$ on the RSRB at 45/135/225/315 degrees azimuth at Mach 0.95 for simplified and detailed booster forward attachment with comparison to fence BMOs.
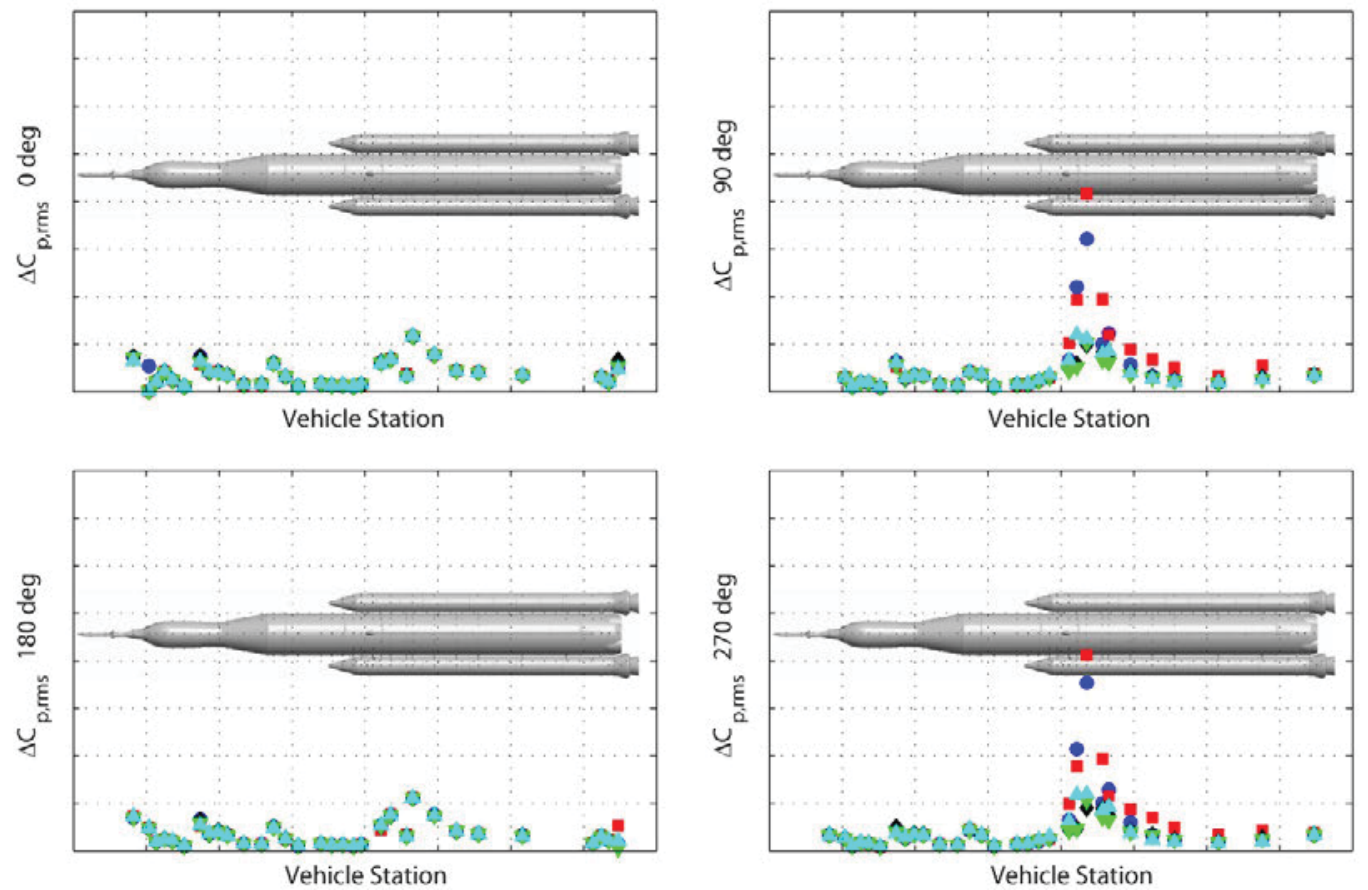

SLS-10005 Core $0 / 90 / 180 / 270 \mathrm{deg}$ Bandpass $0.5-60 \mathrm{~Hz}$

$M=1.10 \quad 0=0$ deg $\phi=0$ deg

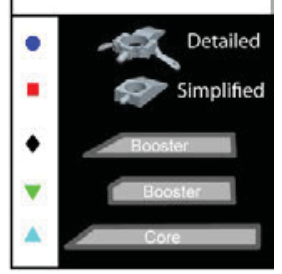

Figure 51. $\Delta C_{p, r m s}$ on the core at 0/90/180/270 degrees azimuth at Mach 1.10 for simplified and detailed booster forward attachment with comparison to fence BMOs. 

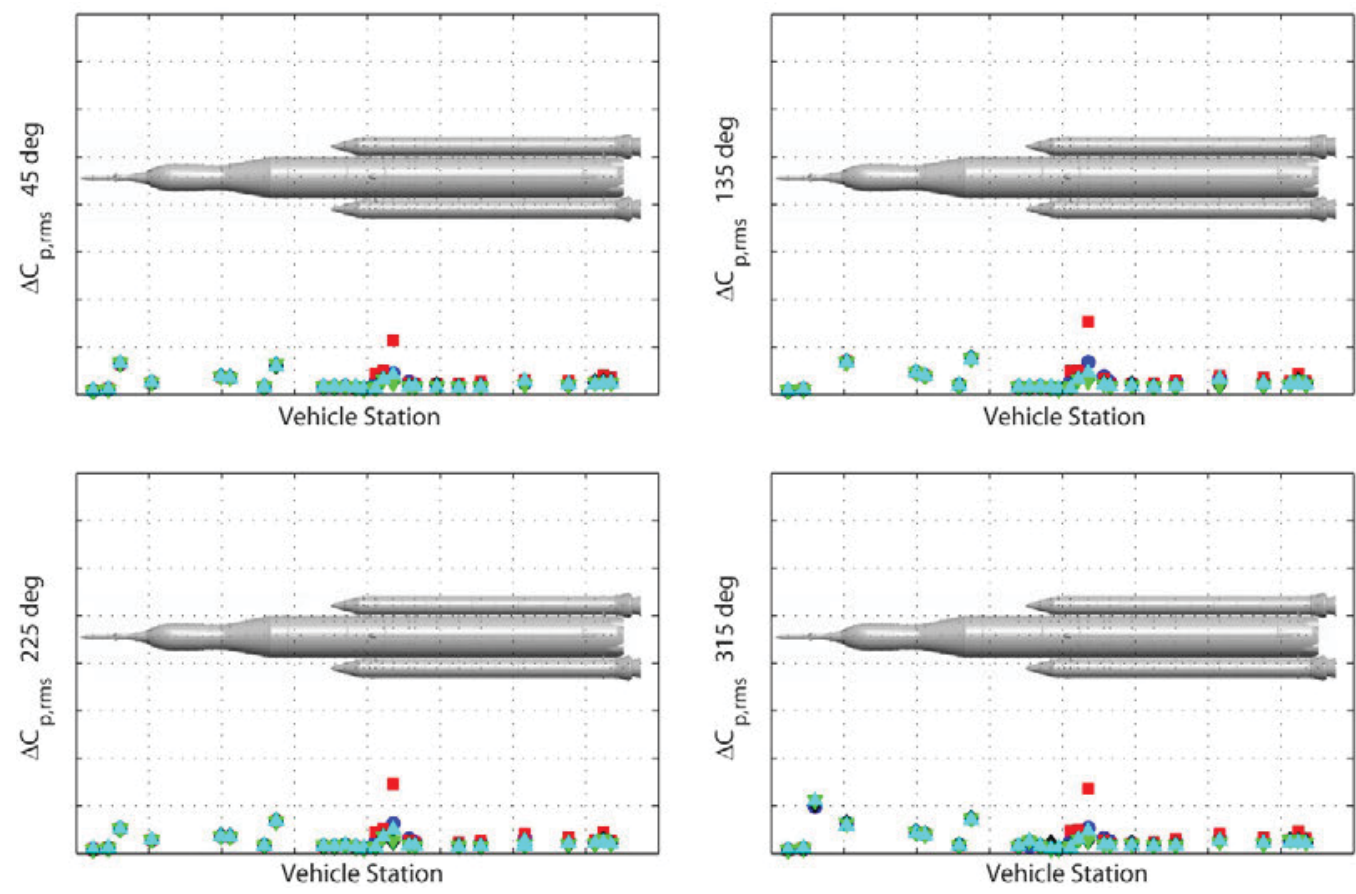

SLS-10005 Core

$45 / 135 / 225 / 315 \mathrm{deg}$

Bandpass $0.5-60 \mathrm{~Hz}$

$M=1.10 \quad \theta=0$ deg $\phi=0$ deg

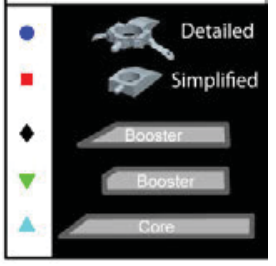

Figure 52. $\Delta C_{p, r m s}$ on the core at 45/135/225/315 degrees azimuth at Mach 1.10 for simplified and detailed booster forward attachment with comparison to fence BMOs.
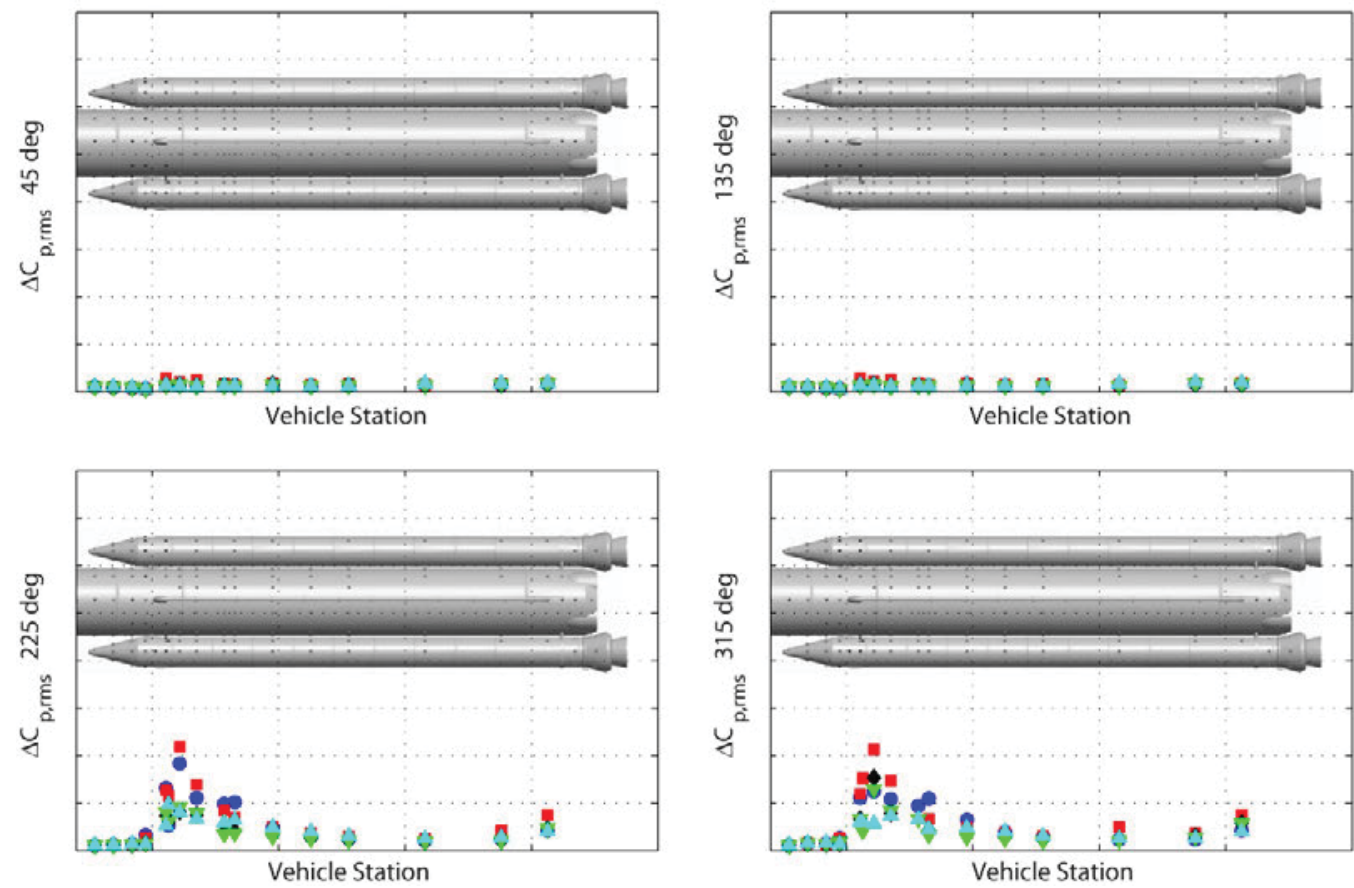

SLS-10005 LSRB

$45 / 135 / 225 / 315$ deg

Bandpass $0.5-60 \mathrm{~Hz}$

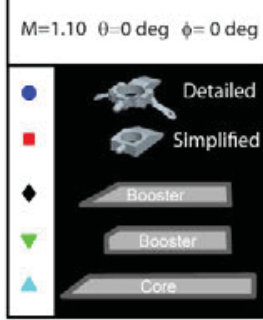

Figure 53. $\Delta C_{p, r m s}$ on the LSRB at 45/135/225/315 degrees azimuth at Mach 1.10 for simplified and detailed booster forward attachment with comparison to fence BMOs. 

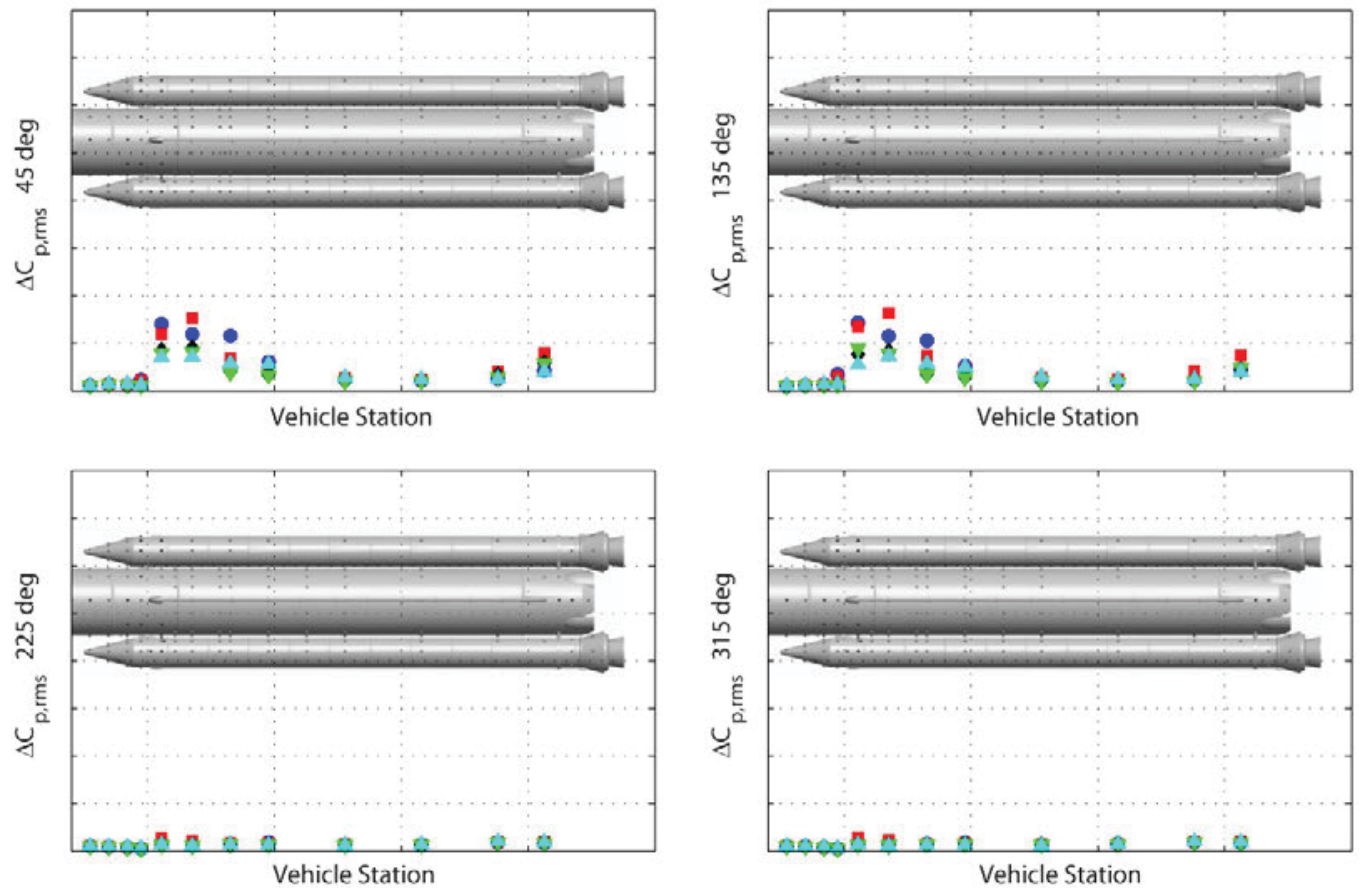

SLS-10005 RSRB $45 / 135 / 225 / 315 \mathrm{deg}$ Bandpass $0.5-60 \mathrm{~Hz}$

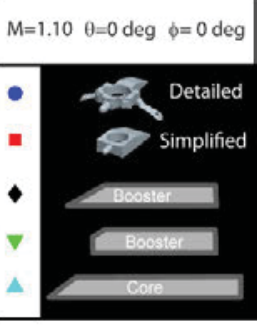

Figure 54. $\Delta C_{p, r m s}$ on the RSRB at 45/135/225/315 degrees azimuth at Mach 1.10 for simplified and detailed booster forward attachment with comparison to fence BMOs.

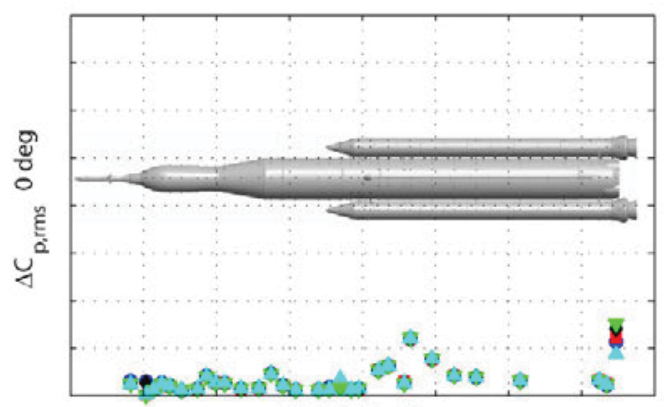

Vehicle Station

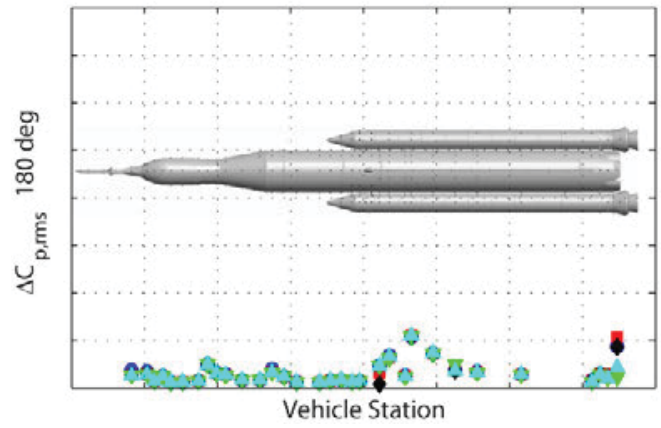

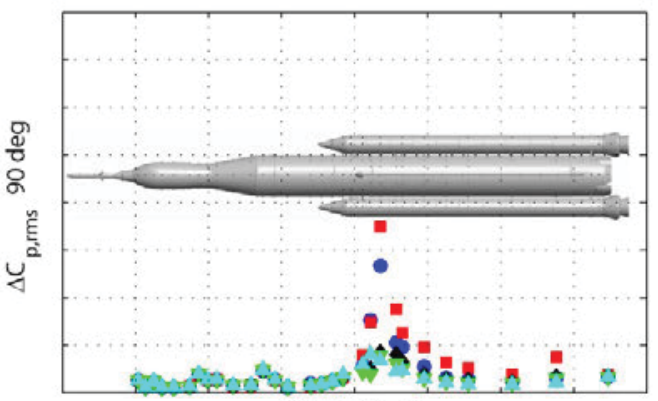

Vehicle Station

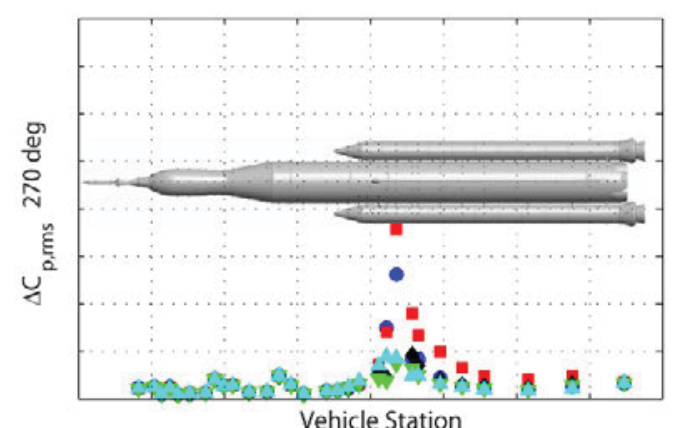

SLS-10005 Core $0 / 90 / 180 / 270 \mathrm{deg}$ Bandpass $0.5-60 \mathrm{~Hz}$

$M=1.20 \quad 0=0$ deg $\phi=0$ deg

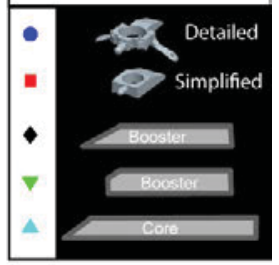

Figure 55. $\Delta C_{p, r m s}$ on the core at 0/90/180/270 degrees azimuth at Mach 1.20 for simplified and detailed booster forward attachment with comparison to fence BMOs. 

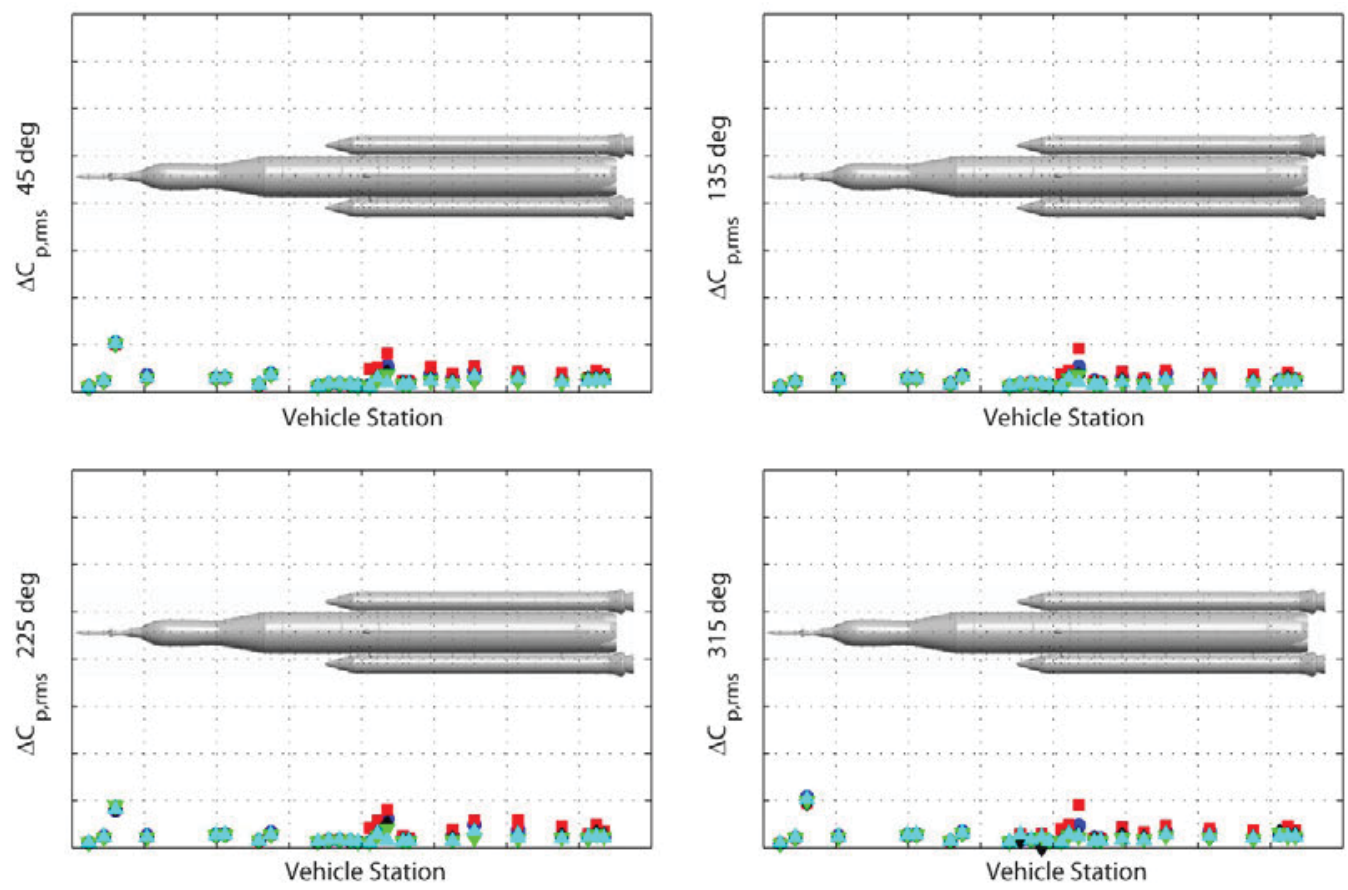

SLS-10005 Core $45 / 135 / 225 / 315 \mathrm{deg}$ Bandpass $0.5-60 \mathrm{~Hz}$

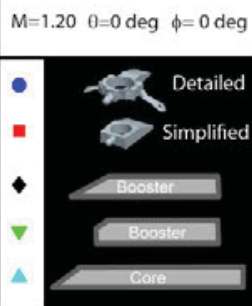

Figure 56. $\Delta C_{p, r m s}$ on the core at 45/135/225/315 degrees azimuth at Mach 1.20 for simplified and detailed booster forward attachment with comparison to fence BMOs.
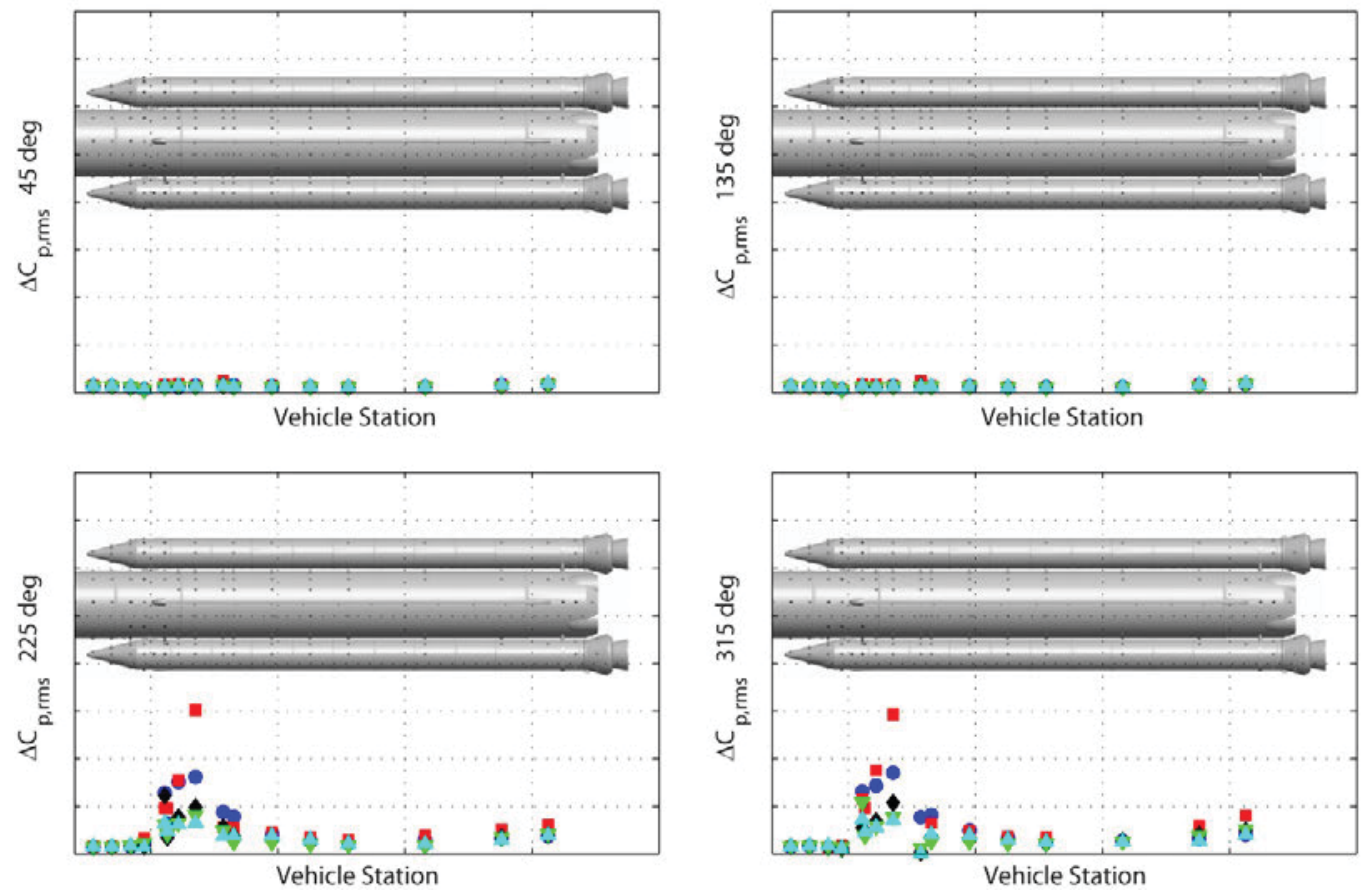

SLS-10005 LSRB $45 / 135 / 225 / 315 \mathrm{deg}$ Bandpass $0.5-60 \mathrm{~Hz}$

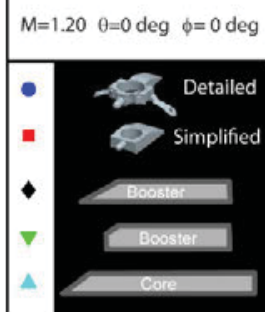

Figure 57. $\Delta C_{p, r m s}$ on the LSRB at 45/135/225/315 degrees azimuth at Mach 1.20 for simplified and detailed booster forward attachment with comparison to fence BMOs. 

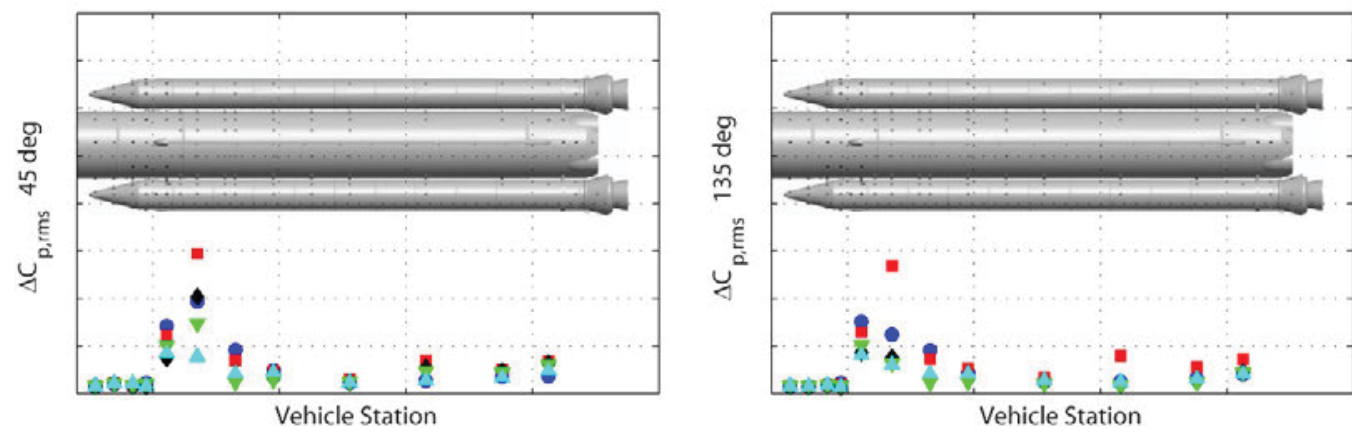

SLS-10005 RSRB $45 / 135 / 225 / 315 \mathrm{deg}$ Bandpass $0.5-60 \mathrm{~Hz}$
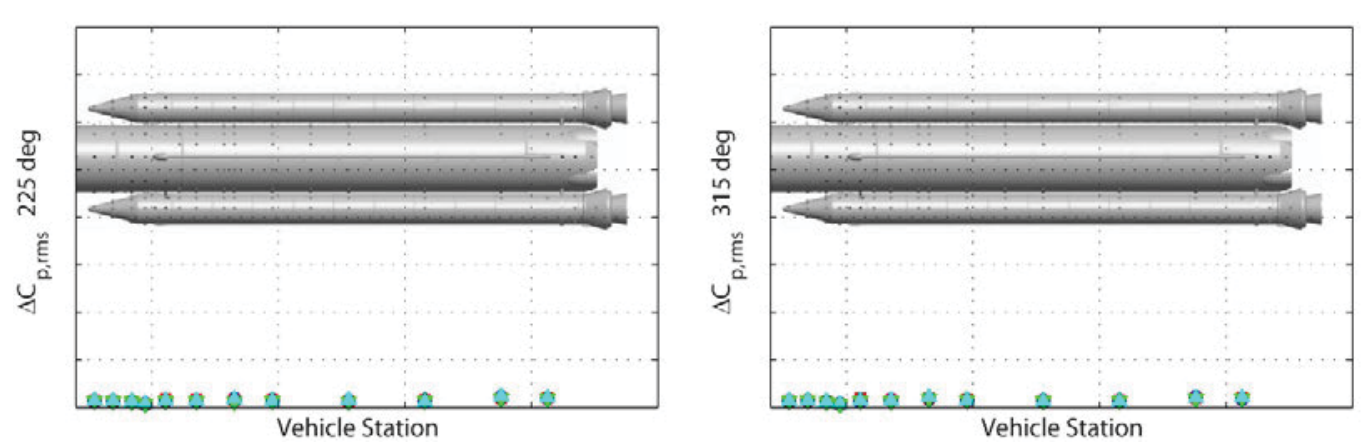

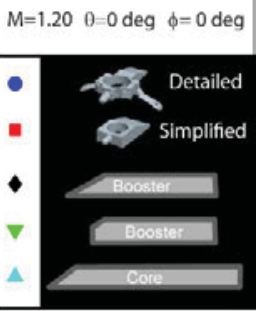

Figure 58. $\Delta C_{p, r m s}$ on the RSRB at 45/135/225/315 degrees azimuth at Mach 1.20 for simplified and detailed booster forward attachment with comparison to fence BMOs.
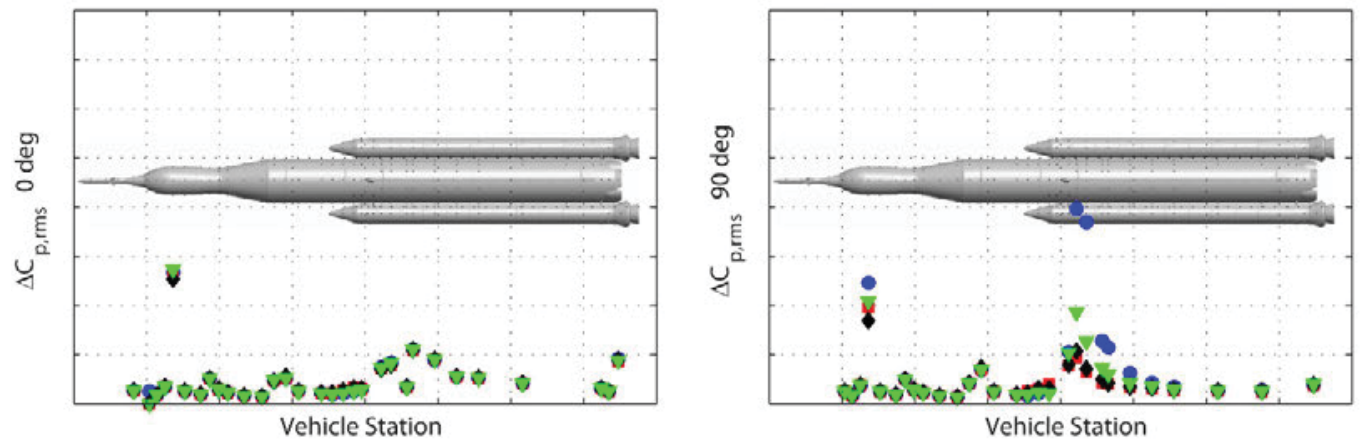

Vehicle Station
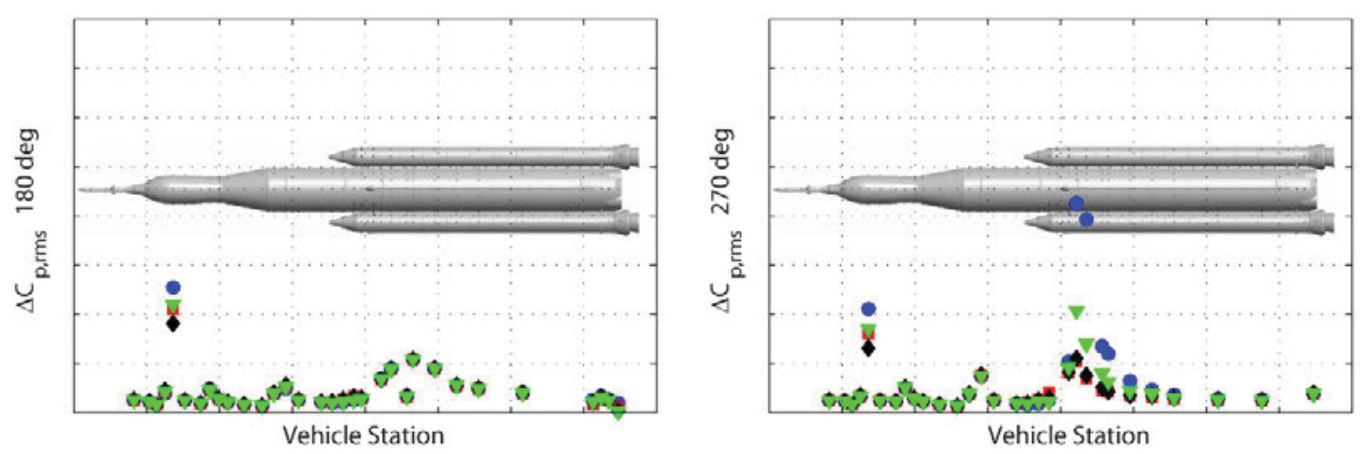

SLS-10005 Core $0 / 90 / 180 / 270 \mathrm{deg}$ Bandpass $0.5-60 \mathrm{~Hz}$

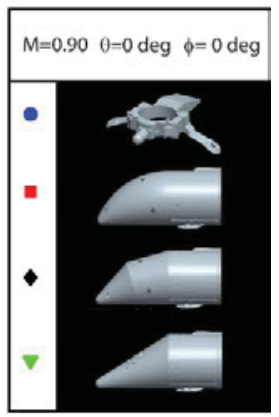

Figure 59. $\Delta C_{p, r m s}$ on the core at $0 / 90 / 180 / 270$ degrees azimuth at Mach 0.90 for detailed booster forward attachment with comparison to booster nose cone BMOs. 

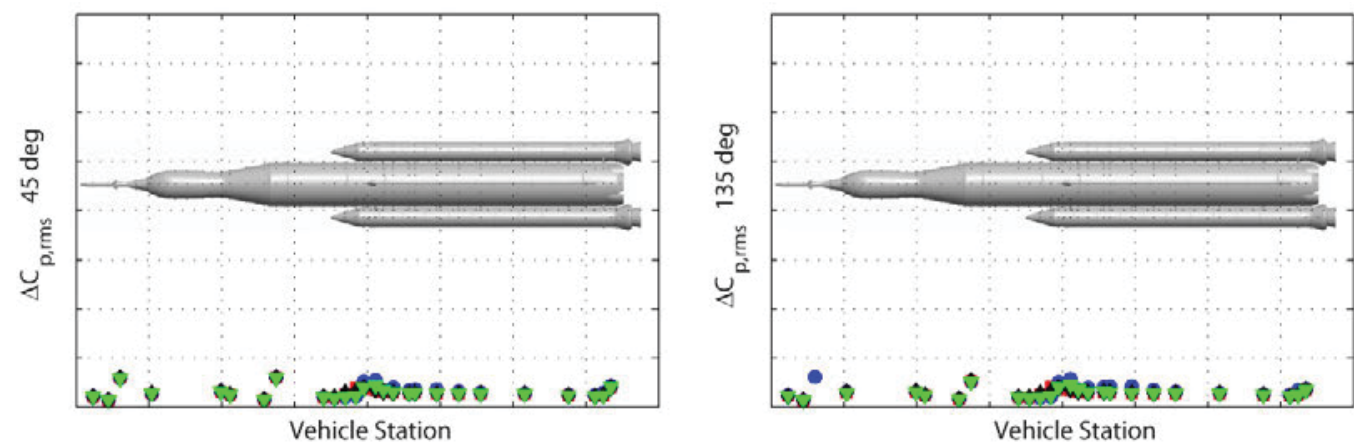

SLS-10005 Core 45/135/225/315 deg Bandpass $0.5-60 \mathrm{~Hz}$
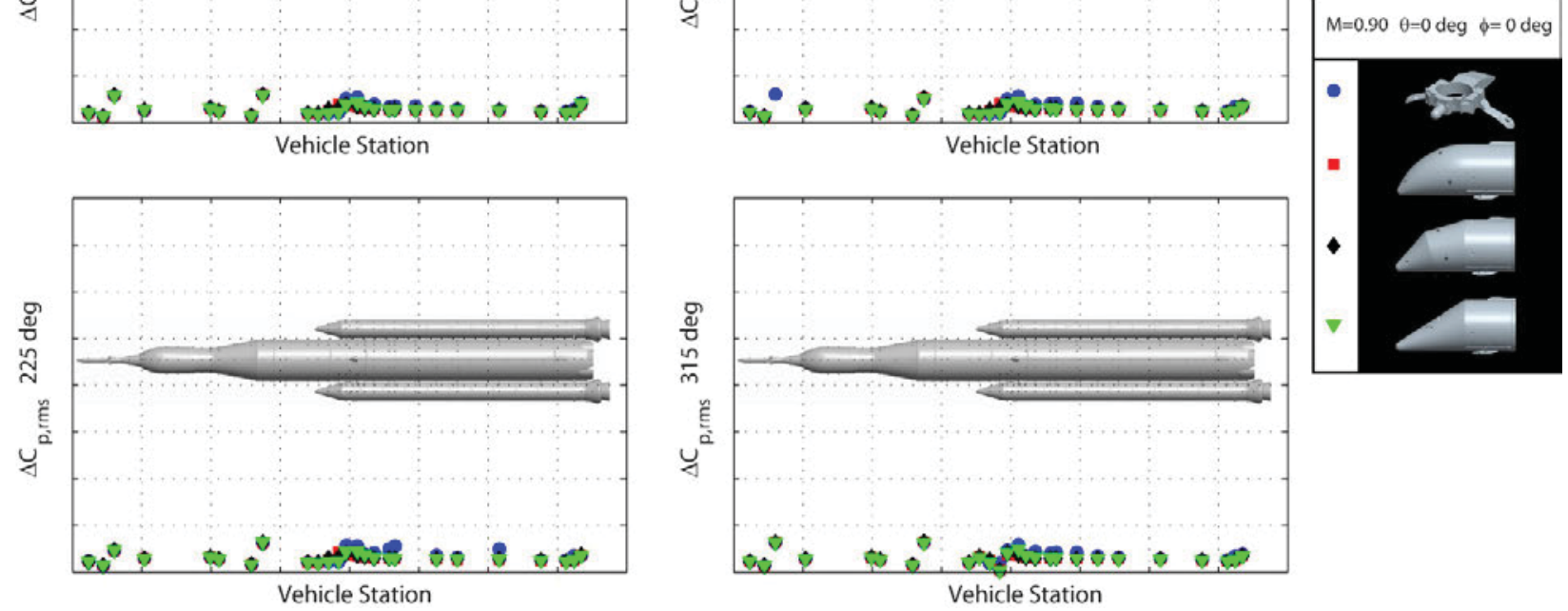

Figure 60. $\Delta C_{p, r m s}$ on the core at 45/135/225/315 degrees azimuth at Mach 0.90 for detailed booster forward attachment with comparison to booster nose cone BMOs.
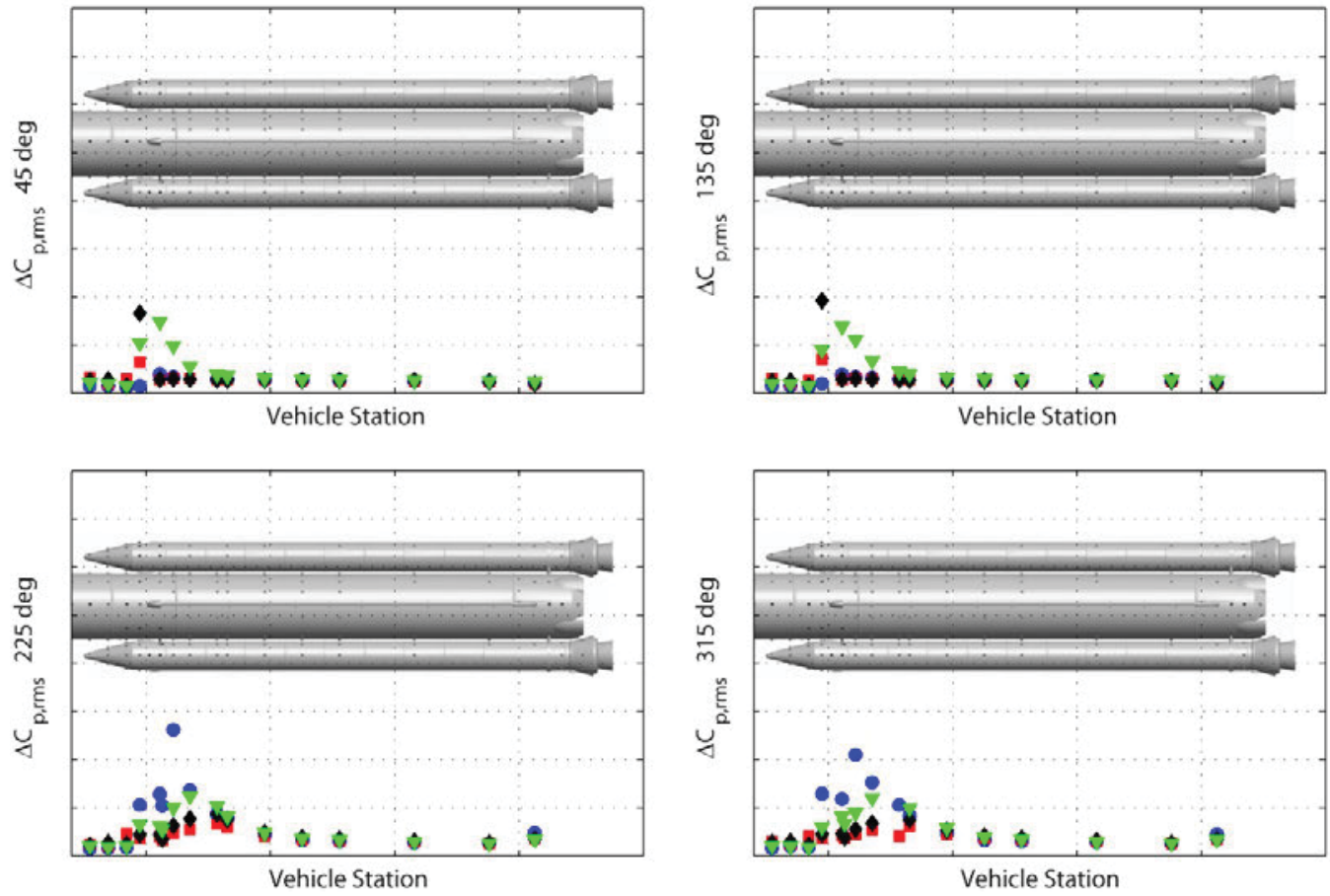

SLS-10005 LSRB $45 / 135 / 225 / 315 \mathrm{deg}$ Bandpass $0.5-60 \mathrm{~Hz}$

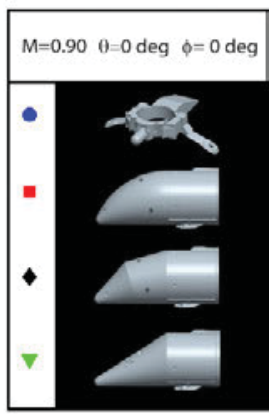

Figure 61. $\Delta C_{p, r m s}$ on the LSRB at 45/135/225/315 degrees azimuth at Mach 0.90 for detailed booster forward attachment with comparison to booster nose cone BMOs. 

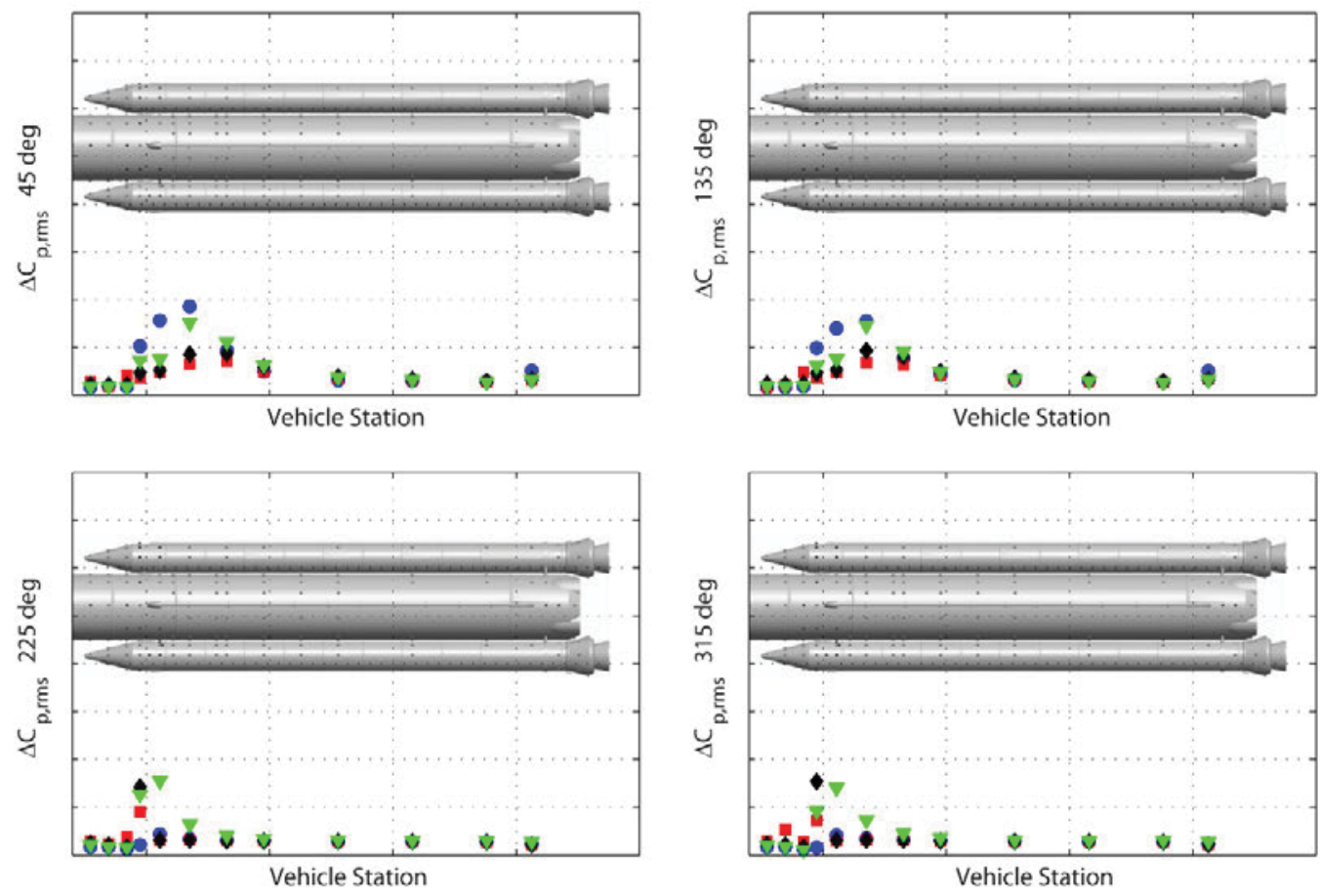

SLS-10005 RSRB $45 / 135 / 225 / 315 \mathrm{deg}$ Bandpass $0.5-60 \mathrm{~Hz}$

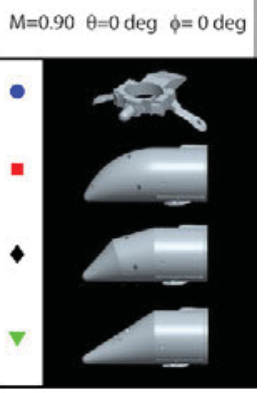

Figure 62. $\Delta C_{p, r m s}$ on the RSRB at 45/135/225/315 degrees azimuth at Mach 0.90 for detailed booster forward attachment with comparison to booster nose cone BMOs.
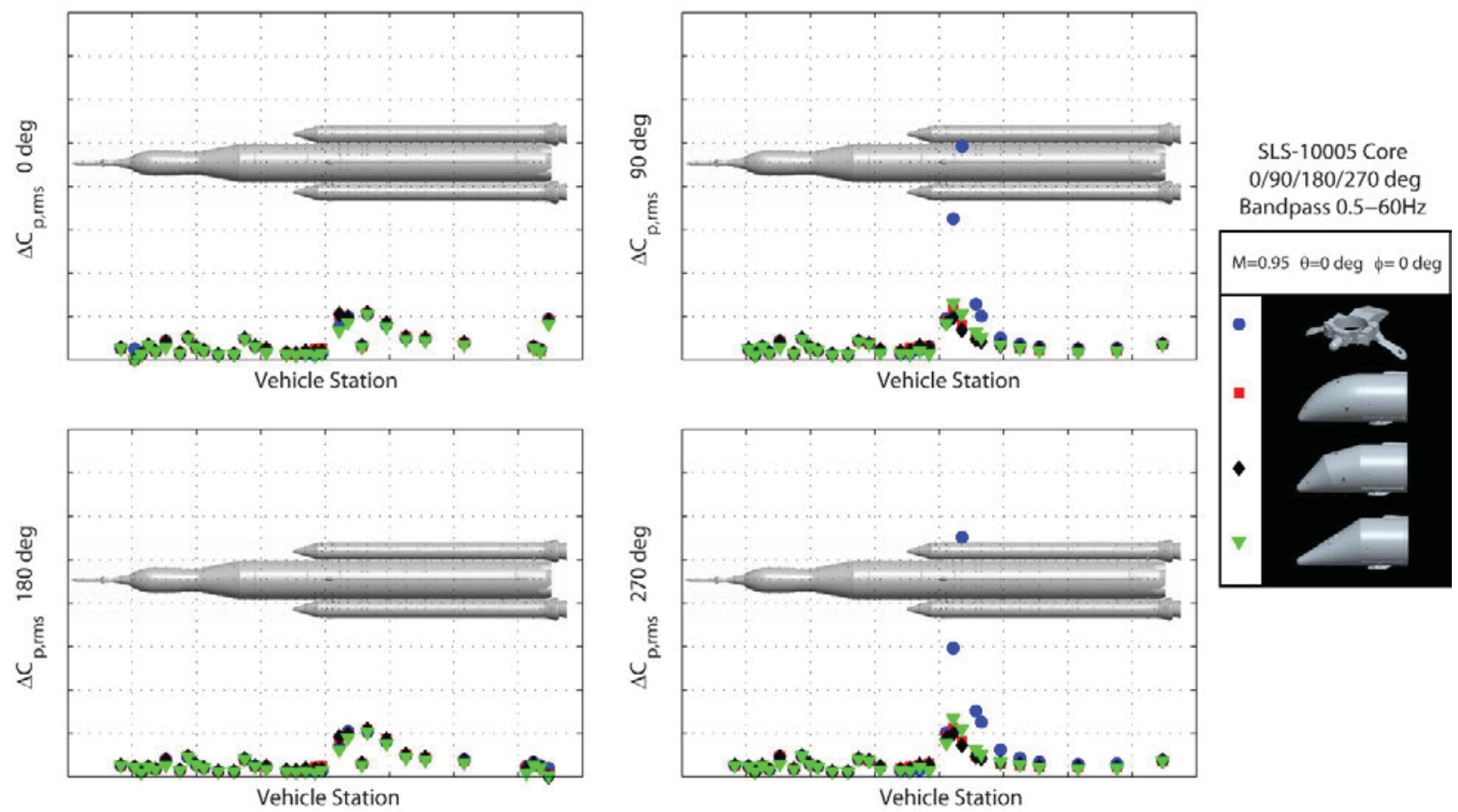

Figure 63. $\Delta C_{p, r m s}$ on the core at 0/90/180/270 degrees azimuth at Mach 0.95 for detailed booster forward attachment with comparison to booster nose cone BMOs. 

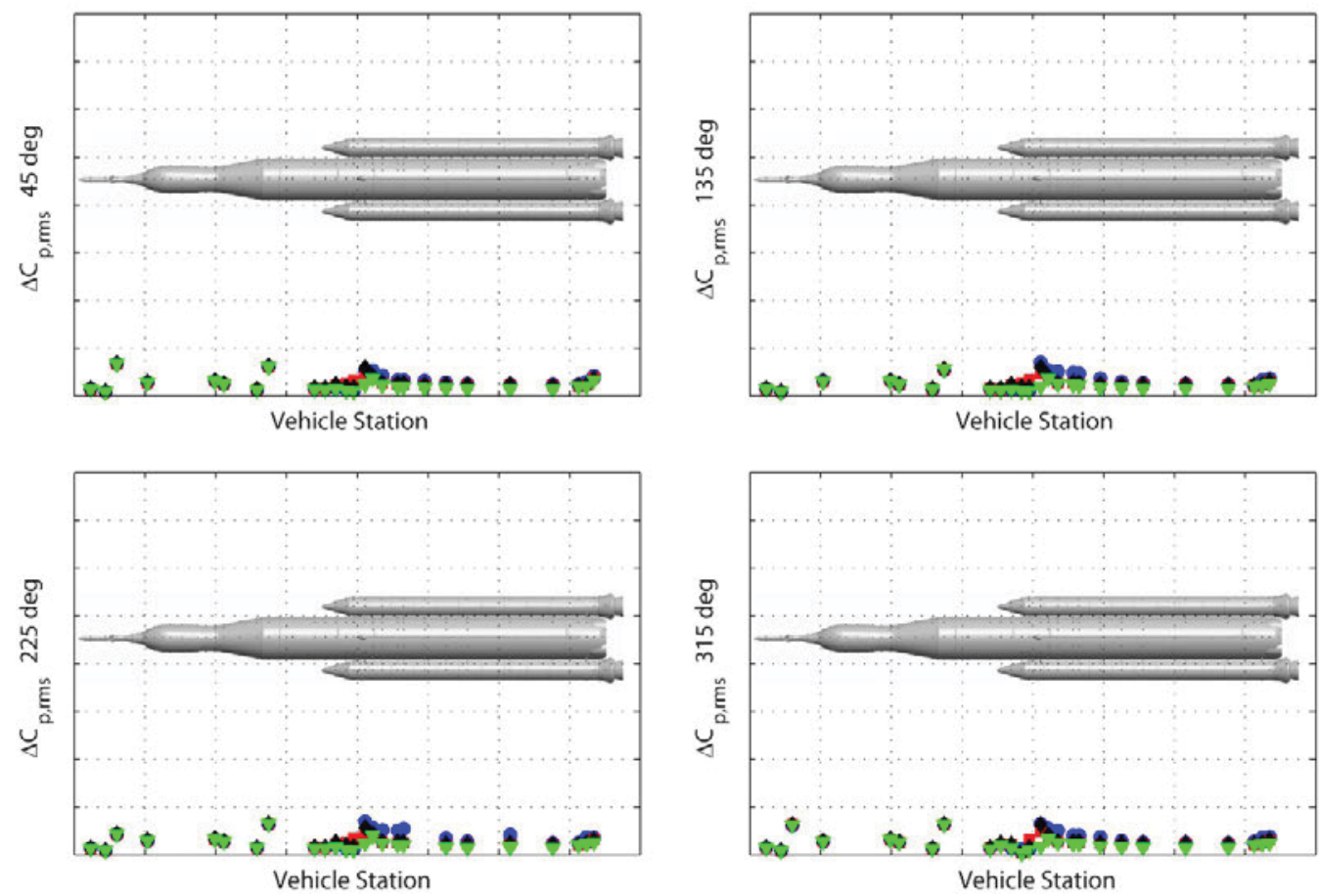

SLS-10005 Core

45/135/225/315 deg

Bandpass $0.5-60 \mathrm{~Hz}$

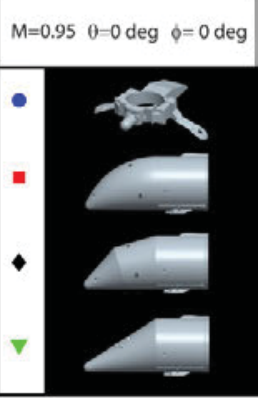

Figure 64. $\Delta C_{p, r m s}$ on the core at 45/135/225/315 degrees azimuth at Mach 0.95 for detailed booster forward attachment with comparison to booster nose cone BMOs.
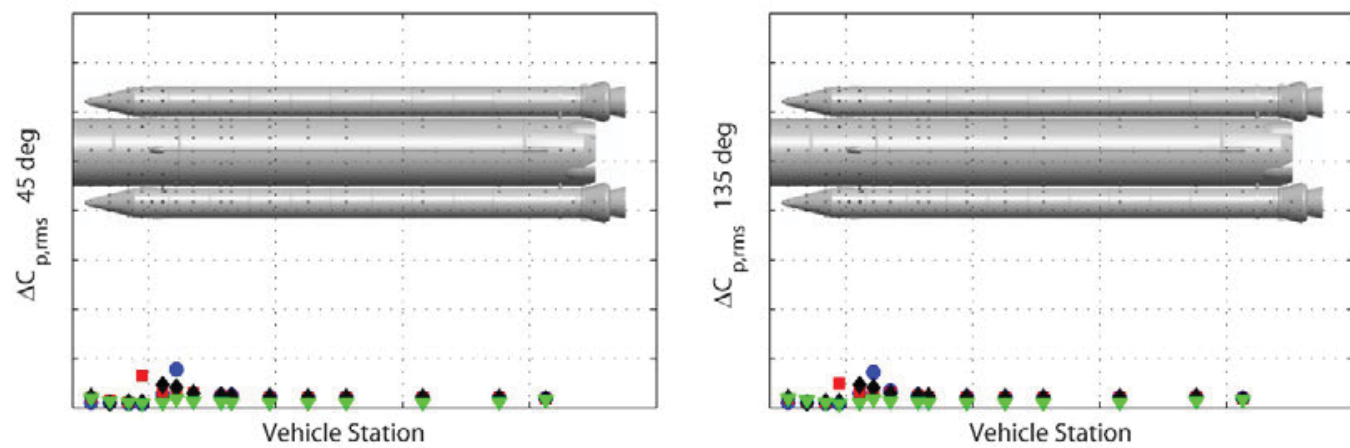

SLS-10005 LSRB $45 / 135 / 225 / 315 \mathrm{deg}$ Bandpass $0.5-60 \mathrm{~Hz}$
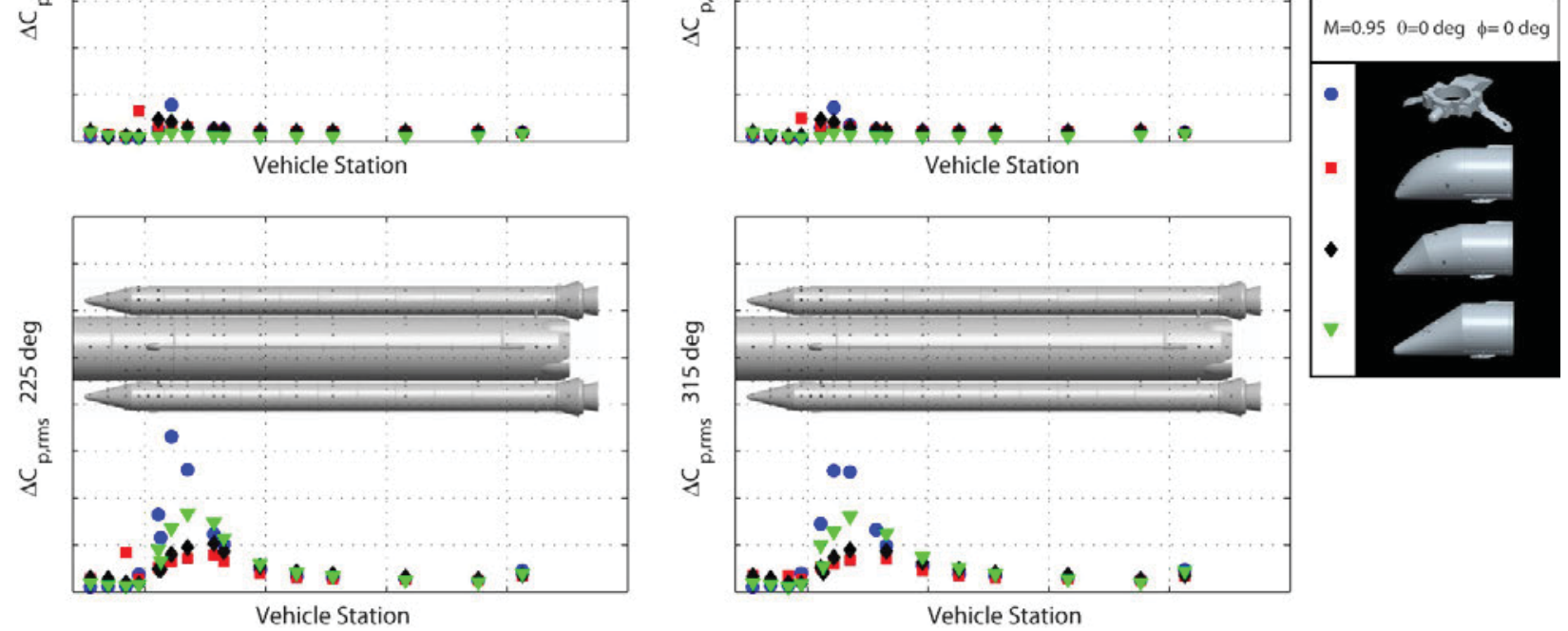

Figure 65. $\Delta C_{p \text {,rms }}$ on the LSRB at 45/135/225/315 degrees azimuth at Mach 0.95 for detailed booster forward attachment with comparison to booster nose cone BMOs. 

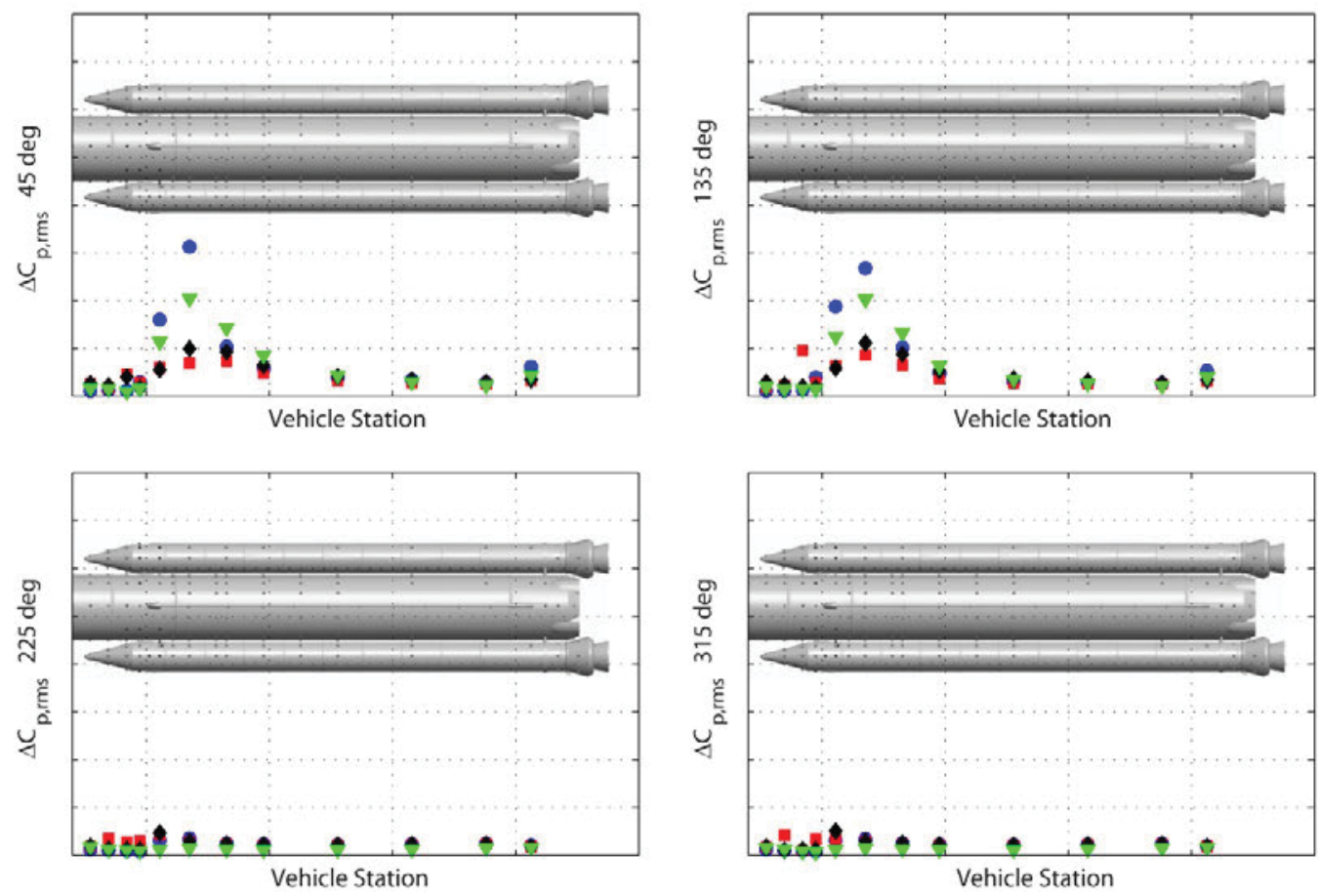

SLS-10005 RSRB $45 / 135 / 225 / 315 \mathrm{deg}$ Bandpass $0.5-60 \mathrm{~Hz}$

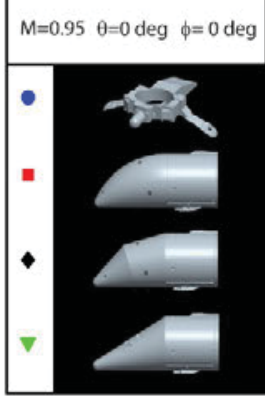

Figure 66. $\Delta C_{p, r m s}$ on the RSRB at 45/135/225/315 degrees azimuth at Mach 0.95 for detailed booster forward attachment with comparison to booster nose cone BMOs.
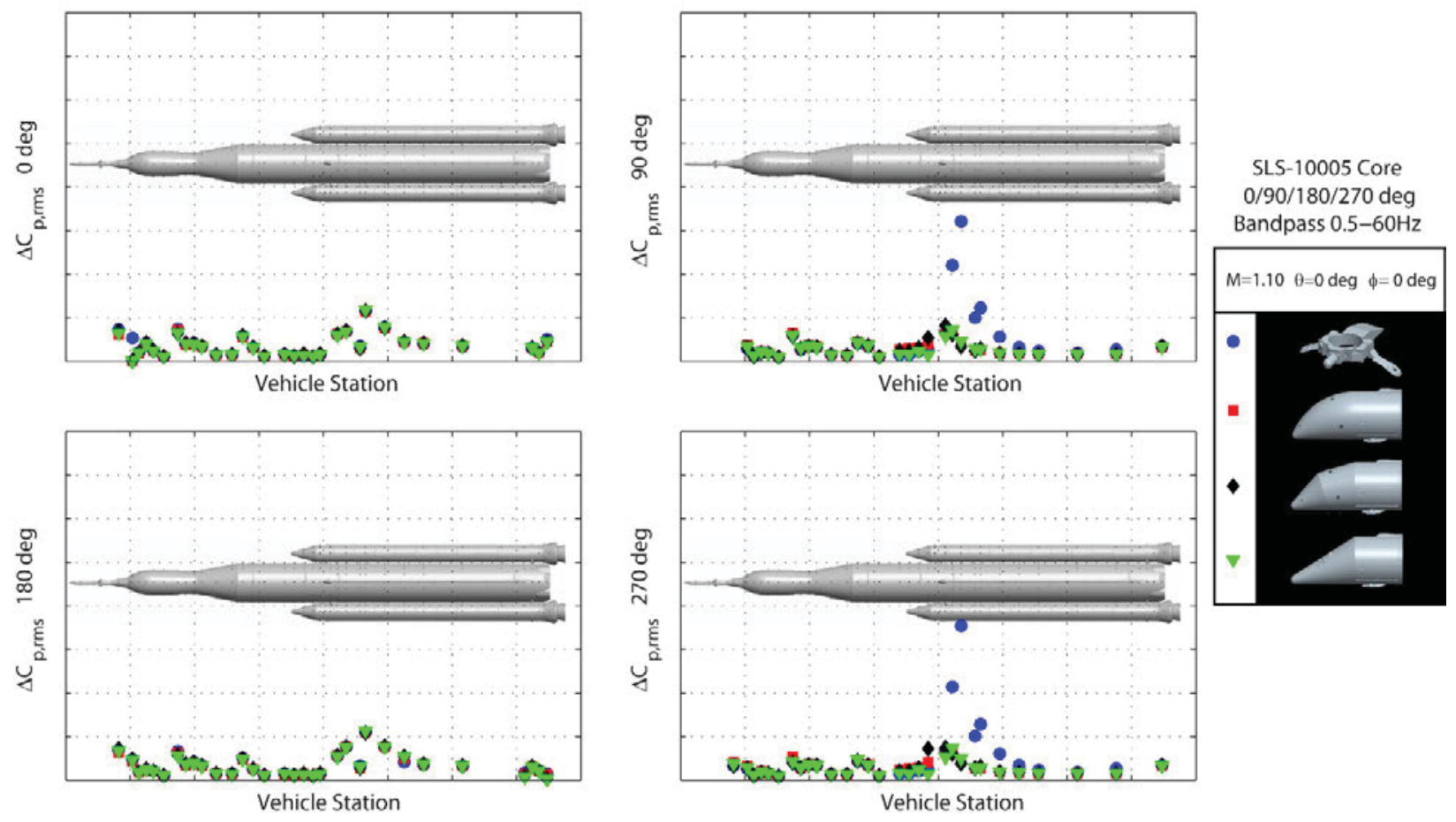

Figure 67. $\Delta C_{p, r m s}$ on the core at 0/90/180/270 degrees azimuth at Mach 1.10 for detailed booster forward attachment with comparison to booster nose cone BMOs. 

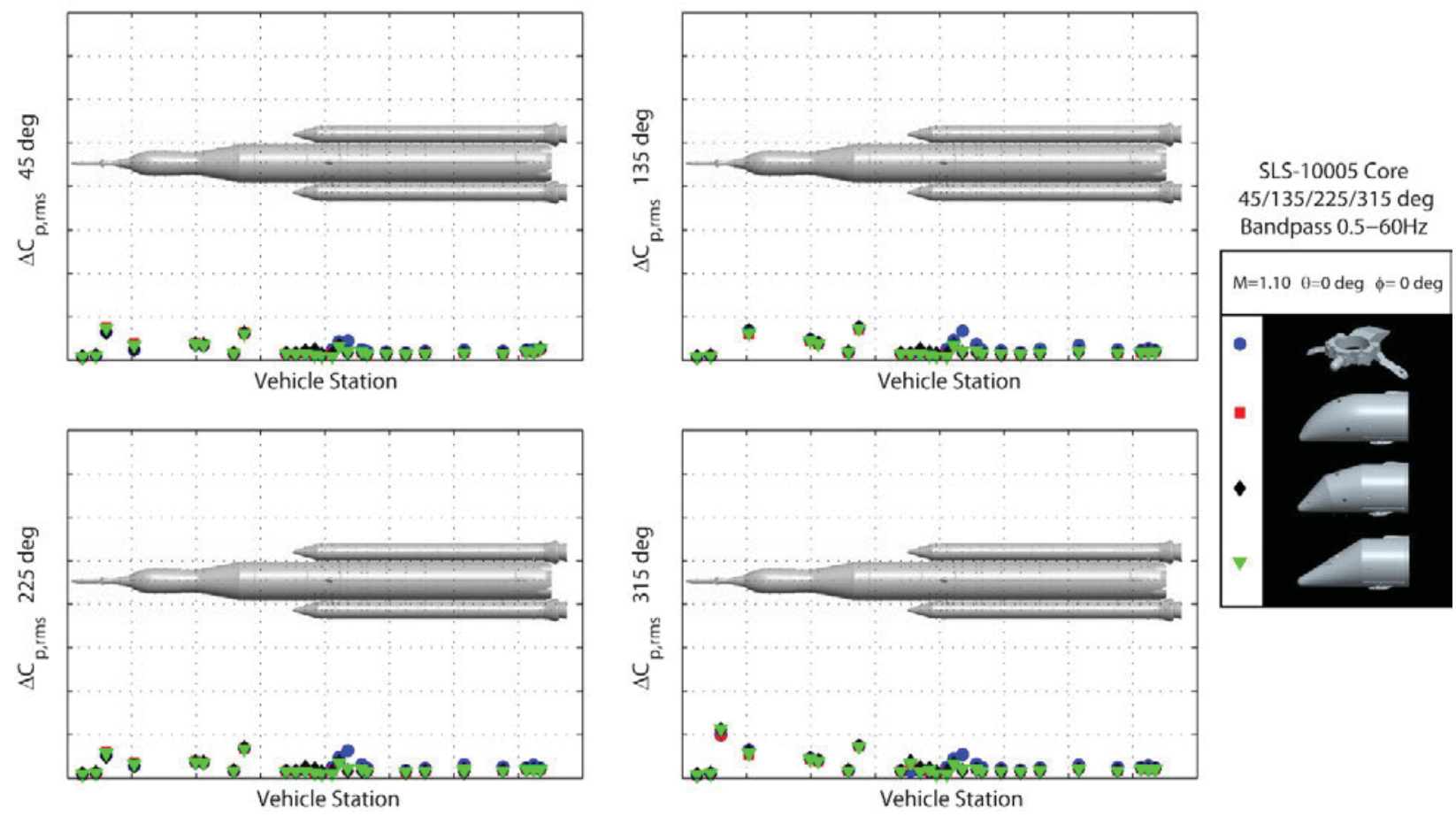

Figure 68. $\Delta C_{p, r m s}$ on the core at 45/135/225/315 degrees azimuth at Mach 1.10 for detailed booster forward attachment with comparison to booster nose cone BMOs.
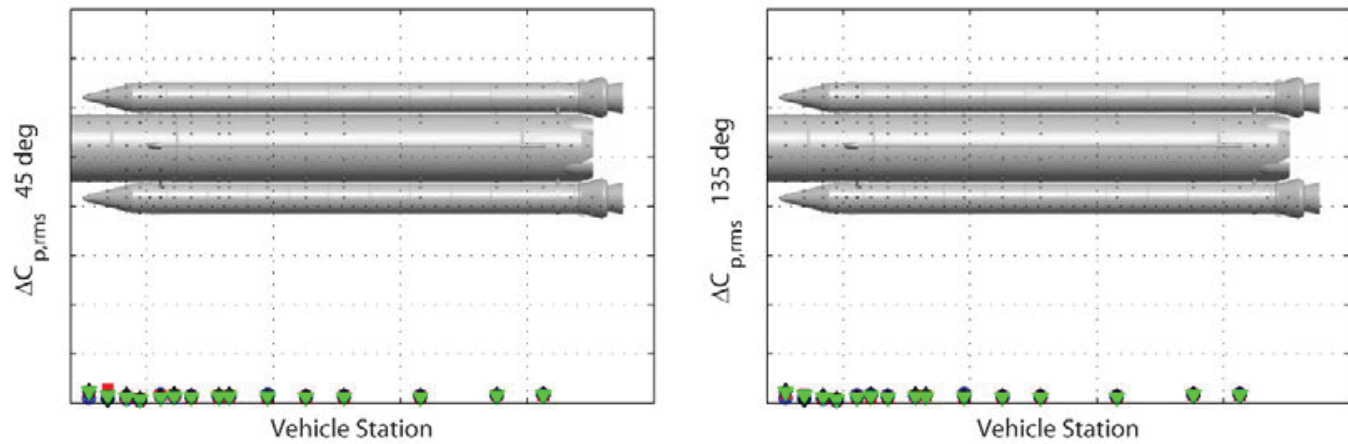

SLS-10005 LSRB $45 / 135 / 225 / 315$ deg Bandpass $0.5-60 \mathrm{~Hz}$
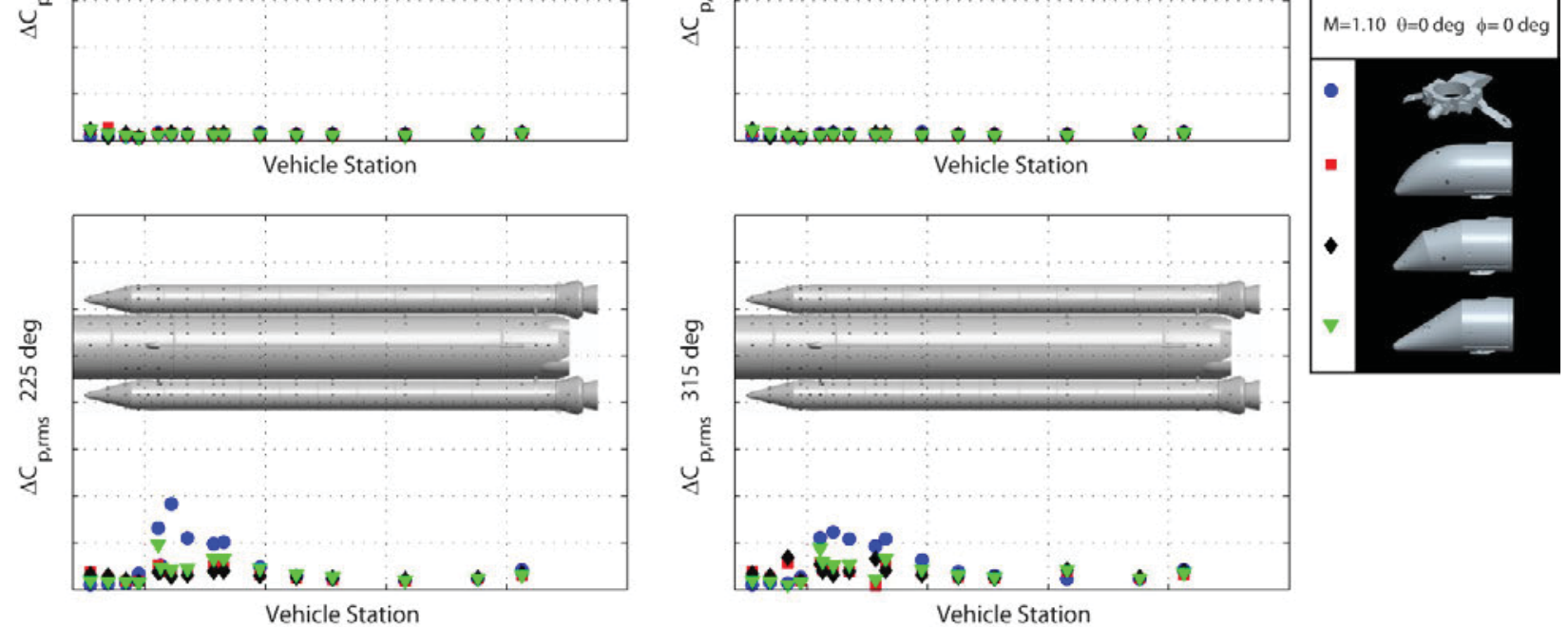

Figure 69. $\Delta C_{p, r m s}$ on the LSRB at 45/135/225/315 degrees azimuth at Mach 1.10 for detailed booster forward attachment with comparison to booster nose cone BMOs. 

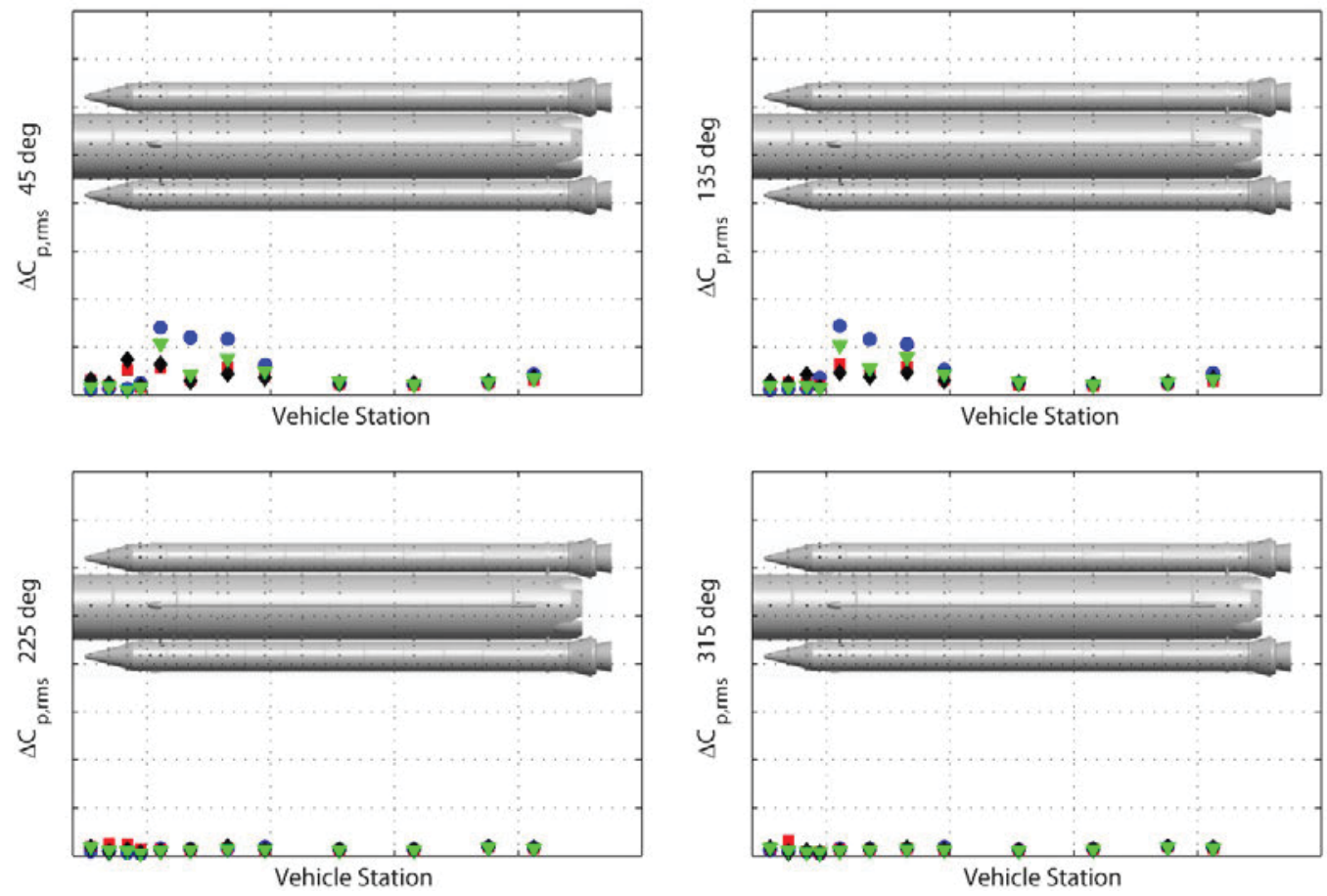

SLS-10005 RSRB $45 / 135 / 225 / 315 \mathrm{deg}$ Bandpass $0.5-60 \mathrm{~Hz}$

$M=1.10 \quad \theta=0 \operatorname{deg} \phi=0$ deg

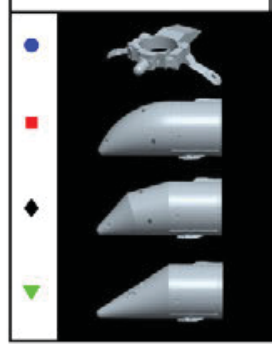

Figure 70. $\Delta C_{p, r m s}$ on the RSRB at 45/135/225/315 degrees azimuth at Mach 1.10 for detailed booster forward attachment with comparison to booster nose cone BMOs.
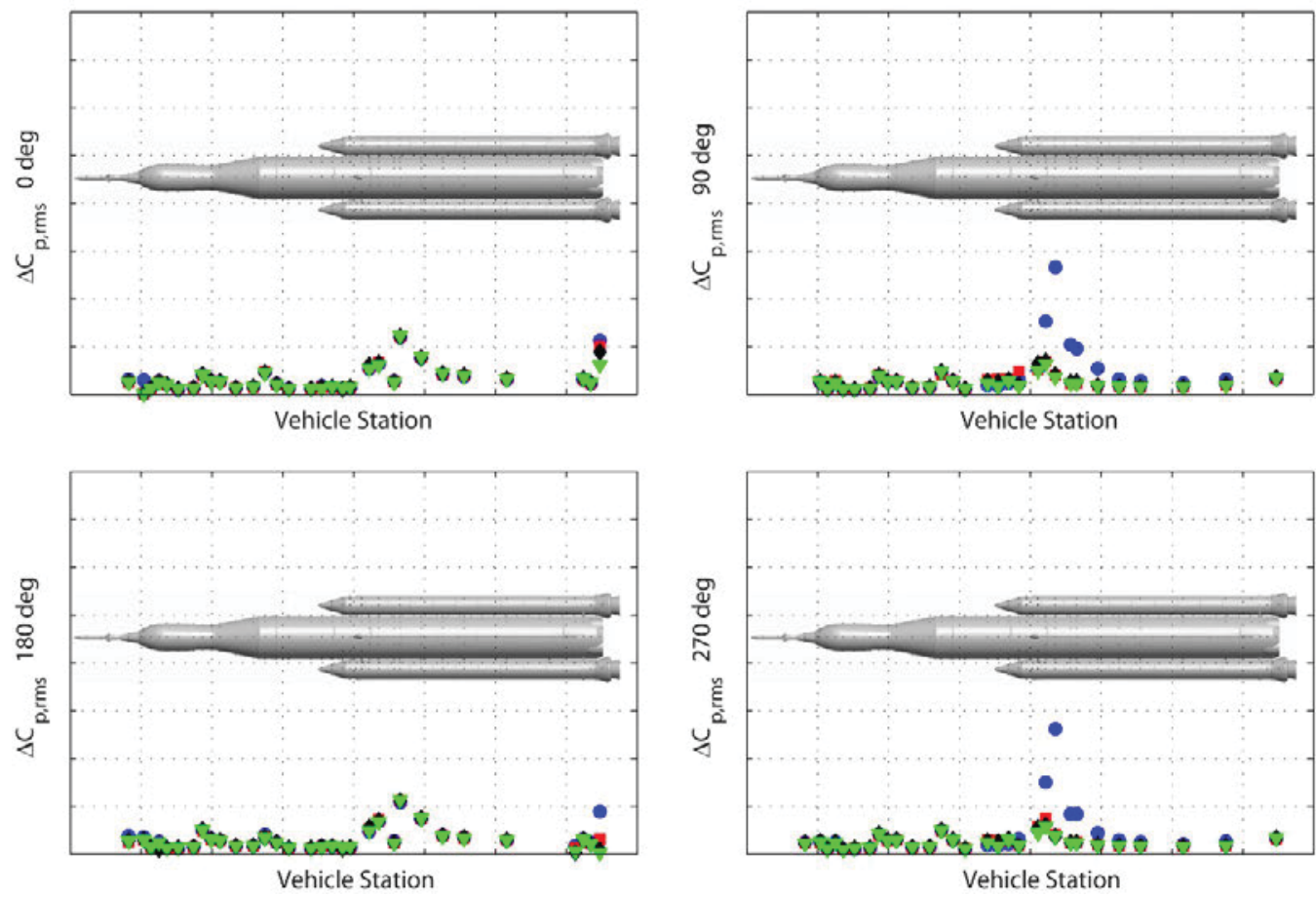

SLS-10005 Core $0 / 90 / 180 / 270 \mathrm{deg}$ Bandpass $0.5-60 \mathrm{~Hz}$

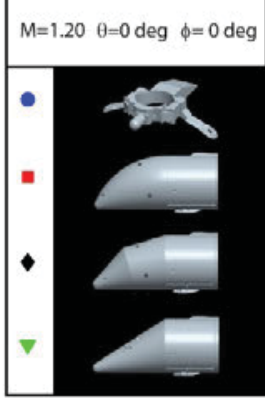

Figure 71. $\Delta C_{p, r m s}$ on the core at 0/90/180/270 degrees azimuth at Mach 1.20 for detailed booster forward attachment with comparison to booster nose cone BMOs. 

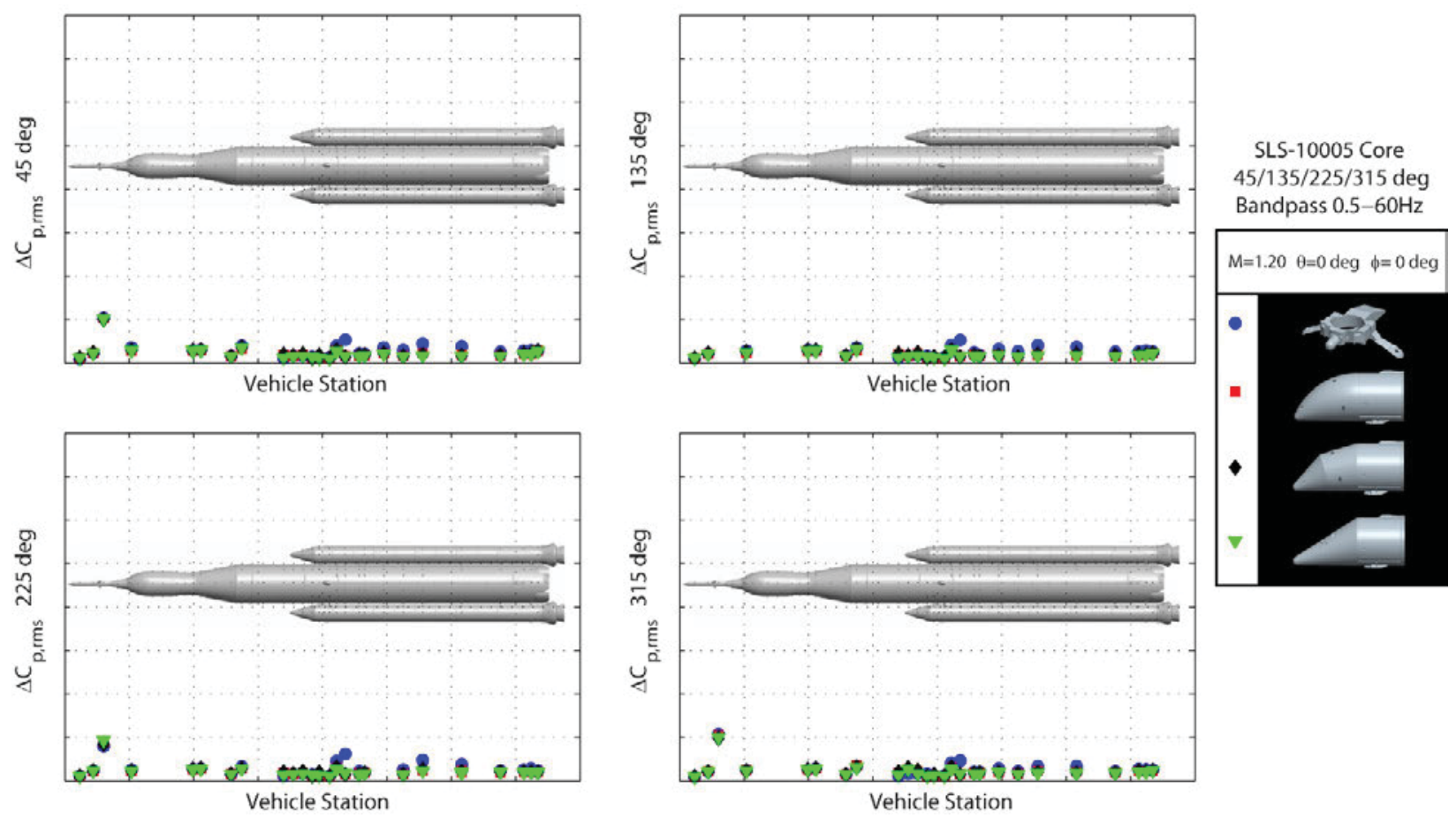

Figure 72. $\Delta C_{p, r m s}$ on the core at 45/135/225/315 degrees azimuth at Mach 1.20 for detailed booster forward attachment with comparison to booster nose cone BMOs.
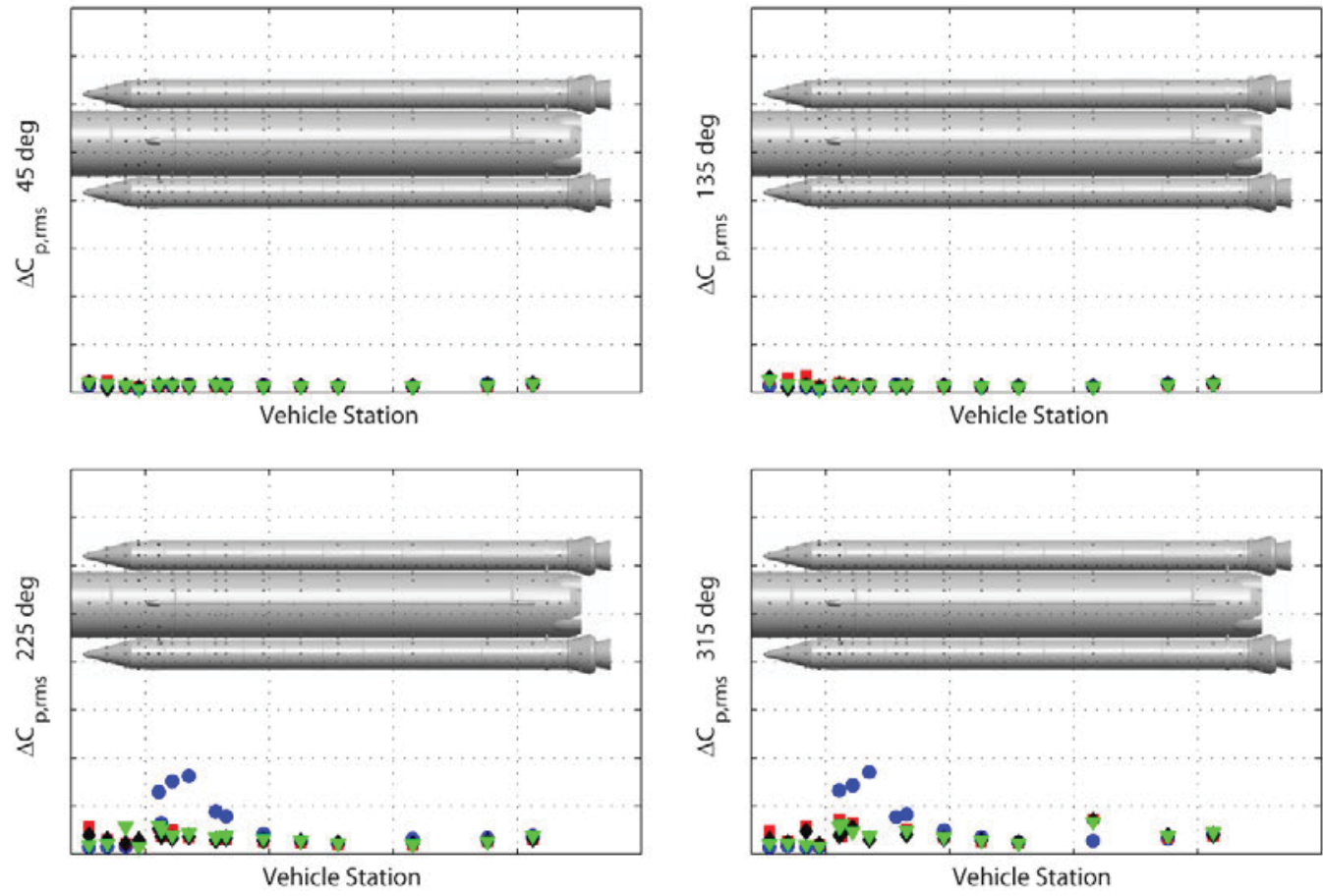

SLS-10005 LSRB $45 / 135 / 225 / 315 \mathrm{deg}$ Bandpass $0.5-60 \mathrm{~Hz}$

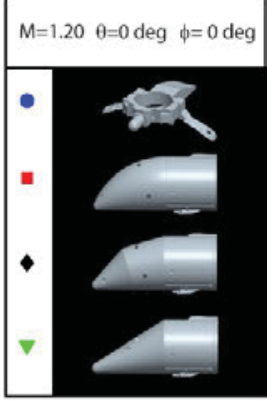

Figure 73. $\Delta C_{p, r m s}$ on the LSRB at $45 / 135 / 225 / 315$ degrees azimuth at Mach 1.20 for detailed booster forward attachment with comparison to booster nose cone BMOs. 

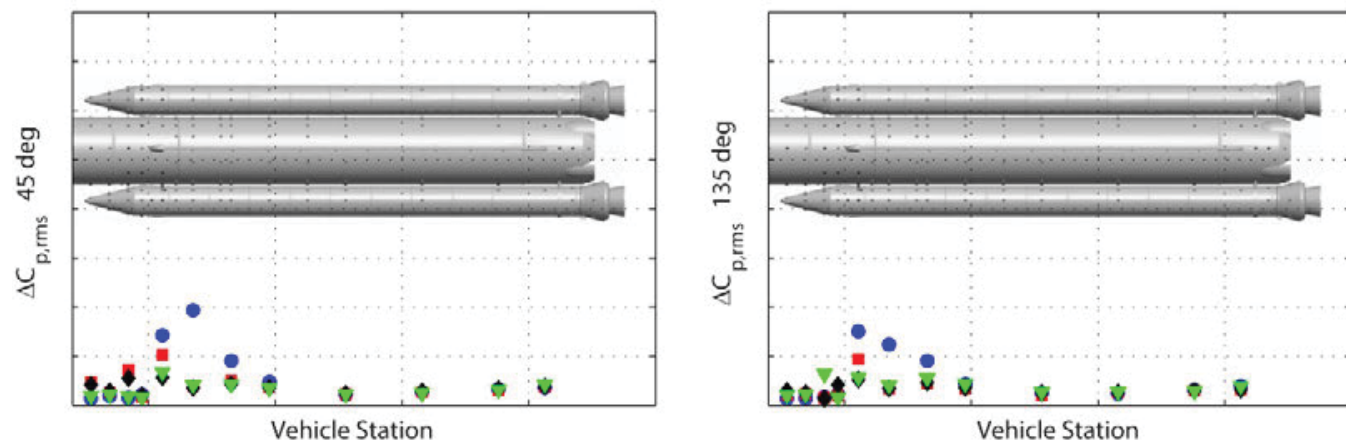

SLS-10005 RSRB $45 / 135 / 225 / 315 \mathrm{deg}$ Bandpass $0.5-60 \mathrm{~Hz}$
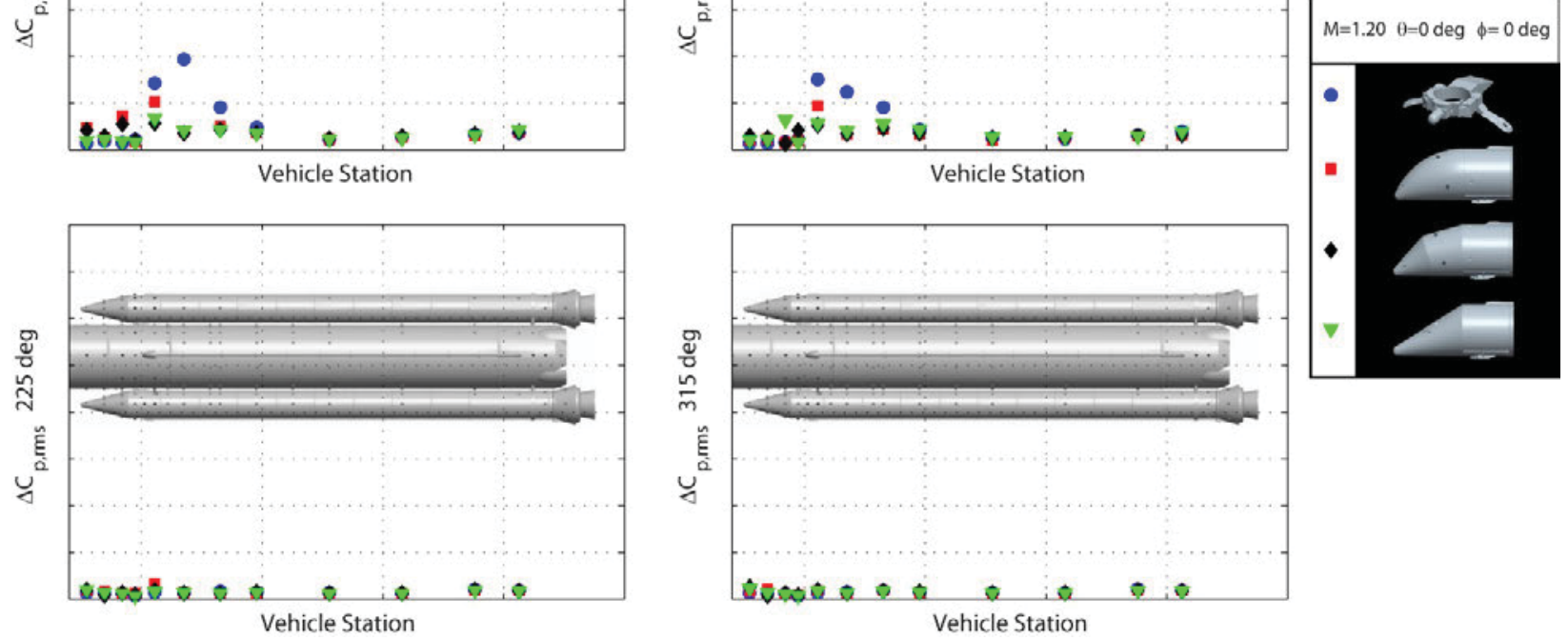

Figure 74. $\Delta C_{p, r m s}$ on the RSRB at 45/135/225/315 degrees azimuth at Mach 1.20 for detailed booster forward attachment with comparison to booster nose cone BMOs. 